\title{
One-Loop Renormalization of Non-Abelian Gauge Theory and $\beta$ Function Based on Loop Regularization Method
}

\author{
Jian-Wei Cui and Yue-Liang Wu \\ Kavli Institute for Theoretical Physics China, Institute of Theoretical Physics \\ Chinese Academy of Science (KITPC/ITP-CAS), Beijing,100080, P.R.China
}

(Dated: January 15, 2008)

\begin{abstract}
All one-loop renormalization constants for Non-Abelian gauge theory are computed in details by using the symmetry-preserving Loop Regularization method proposed in [1, 2]. The resulting renormalization constants are manifestly shown to satisfy Ward-Takahaski-Slavnov-Taylor identities, and lead to the well-known one loop $\beta$ function for Non-Abelian gauge theory of QCD [3]. The loop regularization method is realized in the dimension of original field theories, it maintains not only symmetries but also divergent behaviors of original field theories with the introduction of two energy scales. Such two scales play the roles of characterizing and sliding energy scales as well as ultraviolet and infrared cutoff energy scales. An explicit Check of those identities provides a clear demonstration how the symmetry-preserving Loop Regularization method can consistently be applied to non-Abelian gauge theories.

PACS numbers: 11.10.Gh, 11.15.-q, 11.15.Bt
\end{abstract}

\section{INTRODUCTION}

It is known that quantum field theories (QFTs) can not be defined by the straightforward perturbative expansion because of the ultraviolet (UV) divergences. In order to make meaningful for QFTs, it is necessary to remove infinities from perturbative calculations by renormalizing the fields, masses, and coupling constants. A successful renormalization of QFT was firstly realized in 1940s by Tomonaga [4], Schwinger [5], Feynman[6] and Dyson [7] for the case of QED, while it took until the early of 1970s when Wilson 8] gave it full physical meaning on QFTs.

The first step before renormalization is to modify the behavior of field theory at very large momentum so that all Feynman diagrams become well-defined finite quantities. This procedure is usually called regularization. The most important properties needed for a good regularization method are that it must preserve all symmetries of original field theories and meantime maintain the divergent behavior of original Feynman integrals. In fact, many regularization and renormalization methods have been proposed in the last several decades such as: cut-off regularization 9], Pauli-Villars regularization [10], Schwinger's proper time regularization [11, dimensional regularization 12, lattice regularization 13], constrained differential renormalization [14] and so on. As discussed in 1, 2], each of them has its advantage in applying to different situations. Up to now, there exists no single regularization which is suitable to all purposes in QFTs. In refs. 1, 2], a new symmetry-preserving loop regularization(LR) was introduced to meet the request mentioned above. The key concept in such a new regularization method is the introduction of irreducible loop integrals(ILIs) [1, 2] which are evaluated from Feynman integrals. The gauge symmetry requires a set of necessary and sufficient conditions called consistency conditions [1] which are held between the regularized tensor type ILIs and scalar type ILIs. The loop regularization method was realized to satisfy those consistency conditions [1, 2] in the existence of two energy scales. We shall give a brief introduction for the loop regularization below. For more details on the loop regularization including motivations and concrete computation methods as well as general properties, we refer the original papers [1, 2] to readers. Some interesting applications of this new method have been investigated in [15, 16, 17].

This paper is devoted to explicitly demonstrate how the loop regularization preserves non-Abelian gauge symmetry by evaluating all the renormalization constants at one loop level and verifying the Ward-Takahaski-Slavnov-Taylor identities among the renormalization constants. The paper is organized as follows: in section II, we shape the gauge symmetry into the well-known Ward-Takahaski-Slavnov-Taylor identities, and give the conditions that the renormalization constants must satisfy. In section III, we briefly outline the LR method. In section IV, we explicitly evaluate all the one-loop divergent Feynman diagrams to yield all the renormalization constants of non-Abelian gauge theory by using the loop regularization method, and derive the well-known $\beta$ function [3] once checking manifestly the Ward-Takahaski-Slavnov-Taylor identities among the obtained renormalization constants. The results are found to be consistent with those obtained via the dimensional regularization as the quadratic divergent parts cancel each other due to gauge symmetry. The conclusions and remarks are presented in the last section. 


\section{RENORMALIZATION OF GAUGE THEORY AND WARD-TAKAHASKI-SLAVNOV-TAYLOR IDENTITIES}

The lagrangian of gauge theory with Dirac spinor fields $\psi_{n}\left(n=1, \ldots, N_{f}\right)$ interacting with gauge field $A_{\mu}^{a}(a=$ $\left.1, \ldots, d_{G}\right)$ is:

$$
\mathcal{L}=\bar{\psi}_{n}\left(i \gamma^{\mu} D_{\mu}-m\right) \psi_{n}-\frac{1}{4} F_{\mu \nu}^{a} F^{a \mu \nu}
$$

where:

$$
\begin{aligned}
& F_{\mu \nu}^{a}=\partial_{\mu} A_{\nu}^{a}-\partial_{\nu} A_{\mu}^{a}+g f^{a b c} A_{\mu}^{b} A_{\nu}^{c} \\
& D_{\mu} \psi_{n}=\left(\partial_{\mu}-i g T^{a} A_{\mu}^{a}\right) \psi_{n}
\end{aligned}
$$

According to the Faddeev-Popov [18] quantization method, some ghost fields are necessary to be introduced when fixing a gauge. In the covariant gauge, the lagrangian has the following form:

$$
\begin{aligned}
\mathcal{L}_{e f f}= & \bar{\psi}_{n}\left(i \gamma^{\mu} D_{\mu}-m\right) \psi_{n}-\frac{1}{4} F_{\mu \nu}^{a} F^{a \mu \nu}-\frac{1}{2 \xi}\left(\partial^{\mu} A_{\mu}^{a}\right)^{2}+\partial^{\mu} \bar{c}^{a}\left(\partial_{\mu} \delta^{a c}+g f^{a b c} A_{\mu}^{b}\right) c^{c} \\
= & {\left[\bar{\psi}_{n}\left(i \gamma^{\mu} \partial_{\mu}-m\right) \psi_{n}\right]+\left[-\frac{1}{4}\left(\partial_{\mu} A_{\nu}^{a}-\partial_{\nu} A_{\mu}^{a}\right)^{2}-\frac{1}{2 \xi}\left(\partial^{\mu} A_{\mu}^{a}\right)^{2}\right]+\left[\partial^{\mu} \bar{c}^{a} \delta^{a c} \partial_{\mu} c^{c}\right] } \\
& +g \bar{\psi}_{n} \gamma_{\mu} A^{a \mu} T^{a} \psi_{n}-\frac{1}{2} g f^{a b c}\left(\partial_{\mu} A_{\nu}^{a}-\partial \nu A_{\mu}^{a}\right) A^{b \mu} A^{c \nu}+\frac{1}{4} g^{2} f^{a b c} f^{a d e} A_{\mu}^{b} A_{\nu}^{c} A^{d \mu} A^{e \nu} \\
& +g f^{a b c} \partial^{\mu} \bar{c}^{a} A_{\mu}^{b} c^{c}
\end{aligned}
$$

The corresponding Feynman Rules for this lagrangian are presented in App.B. All one loop Feynman diagrams are shown below (for simplicity, the permutation graphs are omitted):

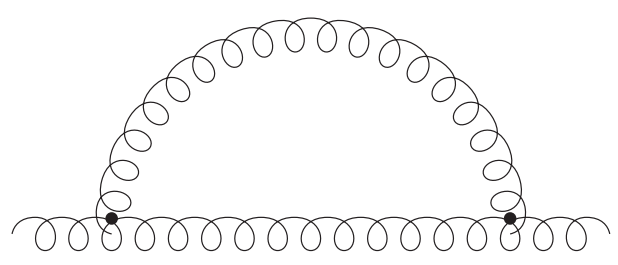

$(1 a)$

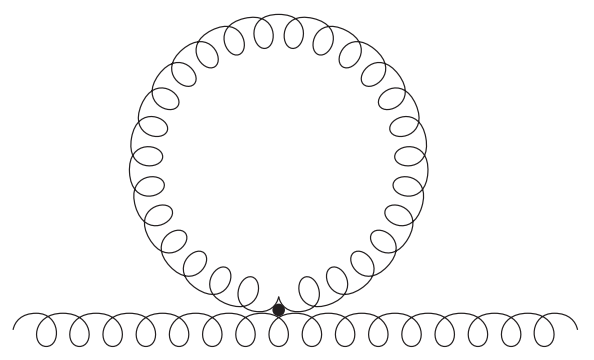

$(1 c)$

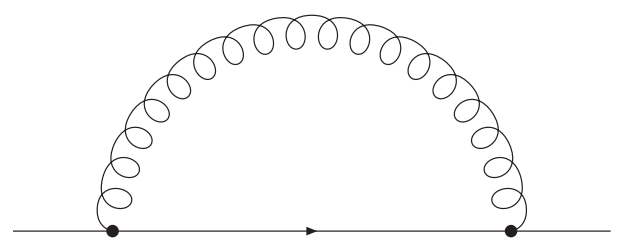

(2)

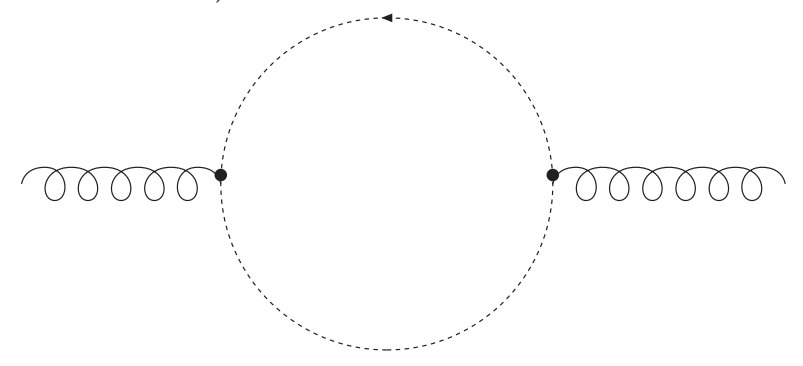

$(1 b)$

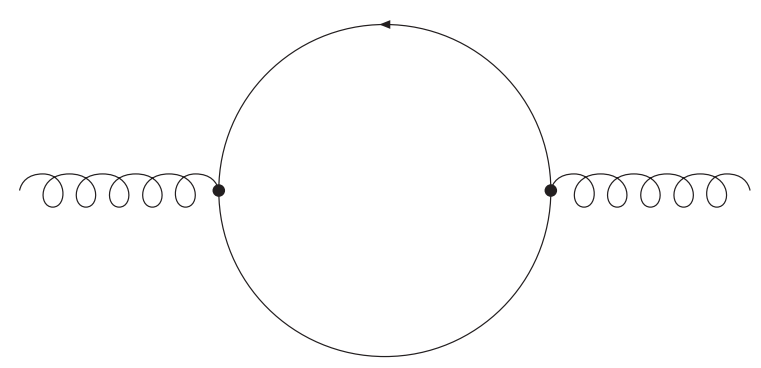

(1d)

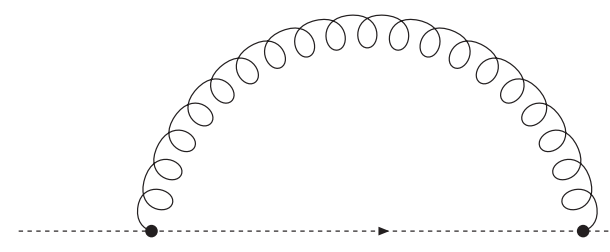

(3) 


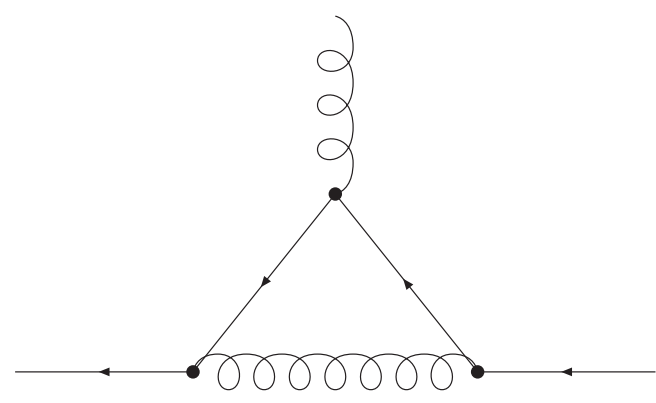

(4a)

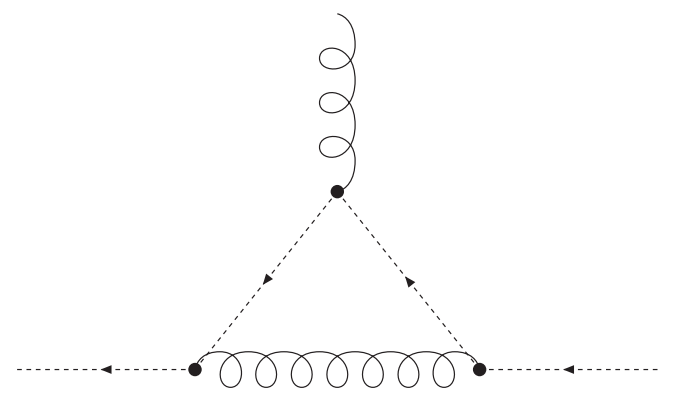

(5a)

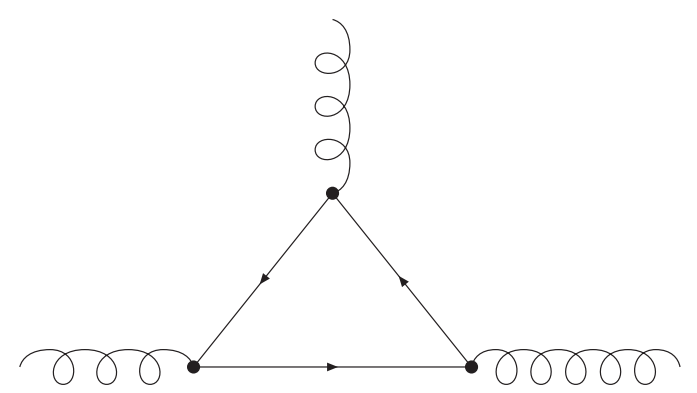

(6a)

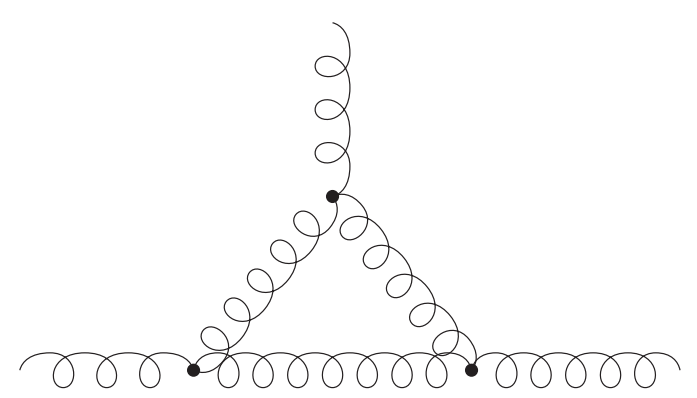

(6c)

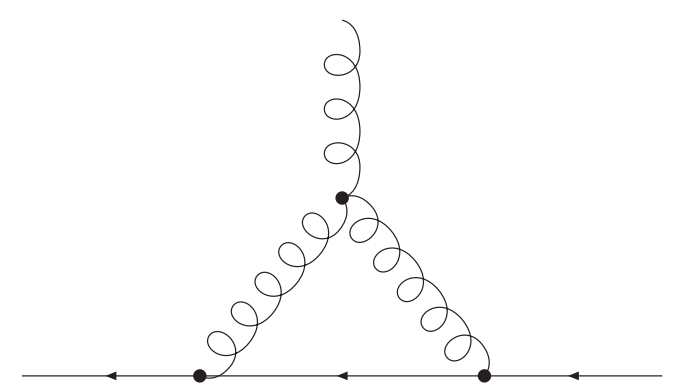

(4b)

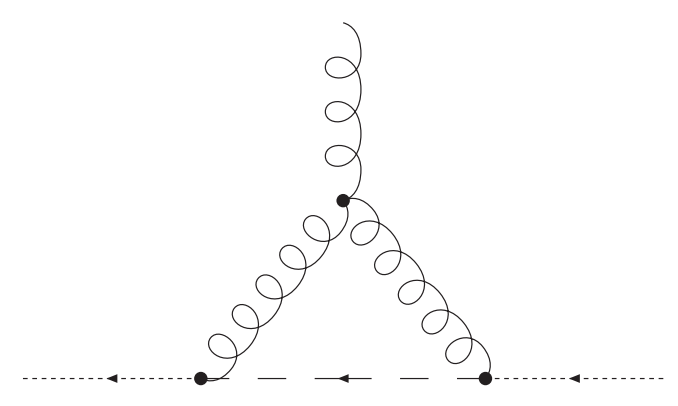

(5b)

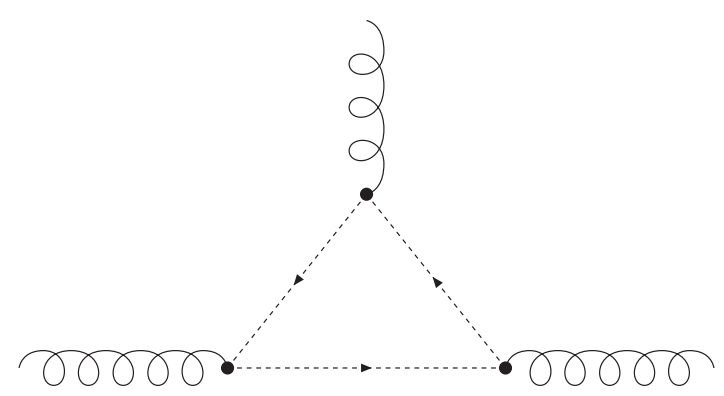

(6b)

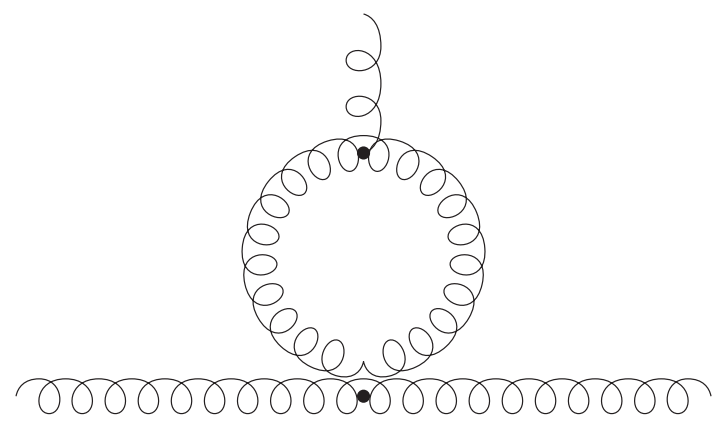

(6d) 


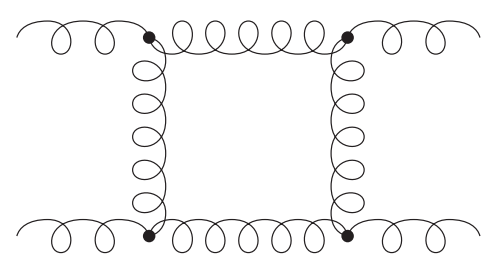

$(7 a)$

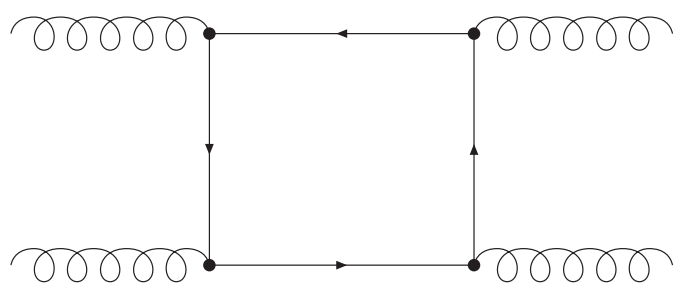

$(7 d)$

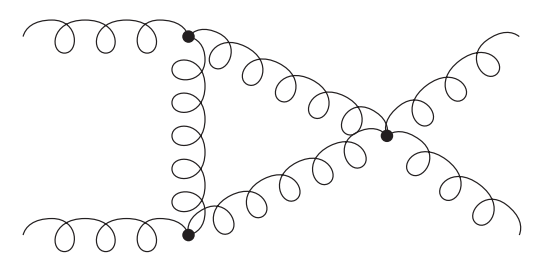

$(7 b)$

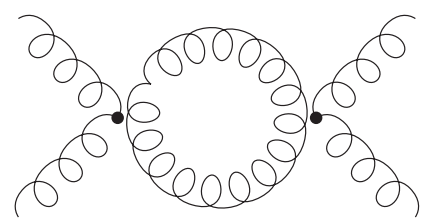

$(7 c)$

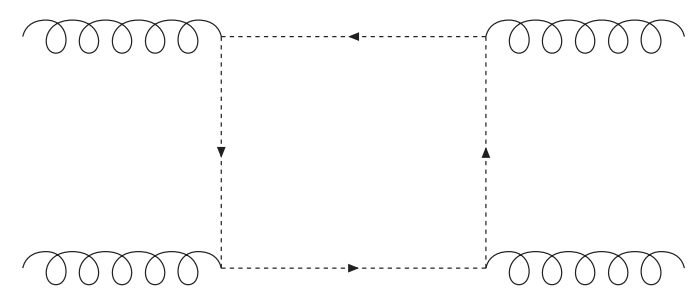

$(7 e)$

Fig.1.

Though all loop diagrams contain divergent integrals, it was proved that gauge theories are renormalizable 19, 20, 21, 22, 23]. To remove the divergence, it is necessary to renormalize the theory by rescaling the fields and redefining the masses and coupling constant. This procedure is equivalent to the introduction of some counterterms to the Lagrangian

$$
\begin{aligned}
\delta \mathcal{L}= & {\left[\left(z_{2}-1\right) \bar{\psi}_{n} i \gamma^{\mu} \partial_{\mu} \psi_{n}-\left(z_{2} z_{m}-1\right) m \bar{\psi}_{n} \psi_{n}\right]+\left(z_{3}-1\right)\left[-\frac{1}{4}\left(\partial_{\mu} A_{\nu}^{a}-\partial_{\nu} A_{\mu}^{a}\right)^{2}\right] } \\
& +\left(\tilde{z}_{3}-1\right)\left[\partial^{\mu} \bar{c}^{a} \delta^{a c} \partial_{\mu} c^{c}\right]+\left(z_{1 F}-1\right) g \bar{\psi}_{n} \gamma_{\mu} A^{a \mu} T^{a} \psi_{n} \\
& -\left(z_{1}-1\right) \frac{1}{2} g f^{a b c}\left(\partial_{\mu} A_{\nu}^{a}-\partial \nu A_{\mu}^{a}\right) A^{b \mu} A^{c \nu}+\left(z_{4}-1\right) \frac{1}{4} g^{2} f^{a b c} f^{a d e} A_{\mu}^{b} A_{\nu}^{c} A^{d \mu} A^{e \nu} \\
& +\left(\tilde{z_{1}}-1\right) g f^{a b c} \partial^{\mu} \bar{c}^{a} A_{\mu}^{b} c^{c}
\end{aligned}
$$

where $z_{1}, \cdots, z_{4}$ are the so-called renormalization constants. They are not independent and must satisfy the relations called Slavnov-Taylor identities [24] which are the generalization of the usual Ward-Takahaski identities. Those identities are actually consequence of the gauge symmetry. To obtain the relations, one can make the BRST transformation 25] which leads to some identities for the generating functional. Then performing a Lengendre transformation we obtained the identities for the 1PI generating functional. Taking the functional derivatives of the 1PI generating functional, one arrives at the relations between the 1PI Green functions. Those relations are the strict restriction of the solution of the gauge symmetry. As a consequence, the renormalization constants should satisfy the following identities [26]:

$$
\frac{z_{1 F}}{z_{3}^{1 / 2} z_{2}}=\frac{\tilde{z}_{1}}{z_{3}^{1 / 2} \tilde{z}_{3}}=\frac{z_{1}}{z_{3}^{3 / 2}}=\frac{z_{4}^{1 / 2}}{z_{3}}
$$

There is a more intuitive method to yield the relations among the renormalization constants. In fact, the gauge independence and the unitarity of the renormalized $\mathrm{S}$ matrix require that the gauge symmetry must be maintained after renormalization [27], which means that the renormalization constants of $g$ obtained from each vertex renormalization must be the same. From such a requirement, one can arrive at above identities. The two-, three- and four-point renormalization constants were evaluated in refs. [30, 31] by using the dimensional regularization. For completeness, we shall perform in this note a detailed calculation for all two-, three- and four-point renormalization constants by using the loop regularization method. As our calculations for the renormalization constants are carried out only at one loop level, which does not involve the renormalization scheme dependence, so we shall not discuss in this note the relevant issues. A detailed discussion on the renormalization scheme prescription in loop regularization will be considered elsewhere. 


\section{BRIEF INTRODUCTION TO LOOP REGULARIZATION METHOD}

In this section we shall briefly introduce the loop regularization method. For our current consideration, we demonstrate only the one loop case. The key concept of the loop regularization is the introduction of irreducible loop integrals (ILIs). It has been shown in 1, 2] that by adopting the Feynman parameterization method with appropriately shifting the integration variables, all one loop Feynman integrals can be expressed in terms of the following 1-fold ILIs:

$$
\begin{aligned}
I_{-2 \alpha} & =\int \frac{d^{4} k}{(2 \pi)^{4}} \frac{1}{\left(k^{2}-M^{2}\right)^{2+\alpha}}, \\
I_{-2 \alpha \mu \nu} & =\int \frac{d^{4} k}{(2 \pi)^{4}} \frac{k_{\mu} k_{\nu}}{\left(k^{2}-M^{2}\right)^{3+\alpha}}, \quad \alpha=-1,0,1,2, \ldots \\
I_{-2 \alpha \mu \nu \rho \sigma} & =\int \frac{d^{4} k}{(2 \pi)^{4}} \frac{k_{\mu} k_{\nu} k_{\rho} k_{\sigma}}{\left(k^{2}-M^{2}\right)^{4+\alpha}}
\end{aligned}
$$

Here $M^{2}$ is in general a function of the external momenta $p_{i}$, the masses of particles $m_{i}$ and the Feynman parameters. Where $I_{2}$ and $I_{0}$ are corresponding to the quadratic and logarithmic divergent integrals.

To maintain the gauge invariance, the regularized 1-fold ILIs should satisfy a set of consistency conditions[1, 2]:

$$
\begin{aligned}
I_{2 \mu \nu}^{R}=\frac{1}{2} g_{\mu \nu} I_{2}^{R}, & I_{2 \mu \nu \rho \sigma}^{R}=\frac{1}{8}\left(g_{\mu \nu} g_{\rho \sigma}+g_{\mu \rho} g_{\nu \sigma}+g_{\mu \sigma} g_{\rho \nu}\right) I_{2}^{R} \\
I_{0 \mu \nu}^{R}=\frac{1}{4} g_{\mu \nu} I_{0}^{R}, & I_{0 \mu \nu \rho \sigma}^{R}=\frac{1}{24}\left(g_{\mu \nu} g_{\rho \sigma}+g_{\mu \rho} g_{\nu \sigma}+g_{\mu \sigma} g_{\rho \nu}\right) I_{0}^{R} .
\end{aligned}
$$

where the superscript " $\mathrm{R} "$ denotes the regularized ILIs.

A simple prescription of loop regularization [1, 2] was realized to ensure the above consistency conditions. The procedure is: Rotating to the four dimensional Euclidean space of momentum, replacing in the ILIs the loop integrating variable $k^{2}$ and the loop integrating measure $\int d^{4} k$ by the corresponding regularized ones $\left[k^{2}\right]_{l}$ and $\int\left[d^{4} k\right]_{l}$ :

$$
\begin{gathered}
k^{2} \rightarrow\left[k^{2}\right]_{l} \equiv k^{2}+M_{l}^{2} \\
\int d^{4} k \rightarrow \int\left[d^{4} k\right]_{l} \equiv \lim _{N, M_{l}^{2}} \sum_{l=0}^{N} c_{l}^{N} \int d^{4} k
\end{gathered}
$$

where $M_{l}^{2}(l=0,1, \cdots)$ may be regarded as the mass factors of loop regulators. If there is no IR divergence in the integrals, one can take the initial conditions $M_{0}^{2}=0$ and $c_{0}^{N}=1$ to recover the original integrals in the limit $M_{l}^{2} \rightarrow \infty$ $(l=1,2, \cdots)$. For IR divergent integrals, one may set $M_{0}^{2}=\mu_{s}^{2}$ to regularize it. The regularized ILIs in the Euclidean space-time are then given by:

$$
\begin{aligned}
I_{-2 \alpha}^{R} & =i(-1)^{\alpha} \lim _{N, M_{l}^{2}} \sum_{l=0}^{N} c_{l}^{N} \int \frac{d^{4} k}{(2 \pi)^{4}} \frac{1}{\left(k^{2}+M^{2}+M_{l}^{2}\right)^{2+\alpha}} \\
I_{-2 \alpha \mu \nu}^{R} & =-i(-1)^{\alpha} \lim _{N, M_{l}^{2}} \sum_{l=0}^{N} c_{l}^{N} \int \frac{d^{4} k}{(2 \pi)^{4}} \frac{k_{\mu} k_{\nu}}{\left(k^{2}+M^{2}+M_{l}^{2}\right)^{3+\alpha}}, \quad \alpha=-1,0,1,2, \ldots \\
I_{-2 \alpha \mu \nu \rho \sigma}^{R} & =i(-1)^{\alpha} \lim _{N, M_{l}^{2}} \sum_{l=0}^{N} c_{l}^{N} \int \frac{d^{4} k}{(2 \pi)^{4}} \frac{k_{\mu} k_{\nu} k_{\rho} k_{\sigma}}{\left(k^{2}+M^{2}+M_{l}^{2}\right)^{4+\alpha}}
\end{aligned}
$$

The coefficients $c_{l}^{N}$ are chosen to satisfy the following conditions:

$$
\lim _{N, M_{l}^{2}} \sum_{l=0}^{N} c_{l}^{N}\left(M_{l}^{2}\right)^{n}=0 \quad(n=0,1, \cdots)
$$

One can easily verify that the following set is the simplest solution of the above conditions:

$$
M_{l}^{2}=\mu_{s}^{2}+l M_{R}^{2}, \quad c_{l}^{N}=(-1)^{l} \frac{N !}{(N-l) ! l !}
$$

Here $M_{R}$ may be regarded as a basic mass scale of loop regulator and the notation $\lim _{N, M_{l}^{2}}$ stands for the limit $\lim _{N, M_{R}^{2} \rightarrow \infty}$. It has been shown in [2] that the above regularization prescription can be understood in terms of 
Schwinger proper time formulation with an appropriate regulating distribution function. Note that the loop regularization is different from the Pauli-Villars regularization in which the regularization prescription is realized through introducing super heavy particles, so that the Pauli-Villars regularization cannot directly be applied to non-Abelian gauge theories. Unlike the Pauli-Villars regularization, the loop regularization is applicable to non-Abelian gauge theories via above regularization prescription on the ILIs.

With the simple solution for $M_{l}^{2}$ and $c_{l}^{N}$ in above equation, the regularized ILIs $I_{0}^{R}$ and $I_{2}^{R}$ can be evaluated explicitly as [1, 2]:

$$
\begin{aligned}
I_{2}^{R} & =\frac{-i}{16 \pi^{2}}\left\{M_{c}^{2}-\mu^{2}\left[\ln \frac{M_{c}^{2}}{\mu^{2}}-\gamma_{w}+1+y_{2}\left(\frac{\mu^{2}}{M_{c}^{2}}\right)\right]\right\} \\
I_{0}^{R} & =\frac{i}{16 \pi^{2}}\left[\ln \frac{M_{c}^{2}}{\mu^{2}}-\gamma_{w}+y_{0}\left(\frac{\mu^{2}}{M_{c}^{2}}\right)\right]
\end{aligned}
$$

with $\mu^{2}=\mu_{s}^{2}+M^{2}$, and

$$
\begin{aligned}
& \gamma_{w} \equiv \lim _{N}\left\{\sum_{l=1}^{N} c_{l}^{N} \ln l+\ln \left[\sum_{l=1}^{N} c_{l}^{N} l \ln l\right]\right\}=\gamma_{E}=0.5772 \cdots, \\
& y_{0}(x)=\int_{0}^{x} d \sigma \frac{1-e^{-\sigma}}{\sigma}, \quad y_{1}(x)=\frac{e^{-x}-1+x}{x} \\
& y_{2}(x)=y_{0}(x)-y_{1}(x), \quad \lim _{x \rightarrow 0} y_{i}(x) \rightarrow 0, i=0,1,2 \\
& M_{c}^{2} \equiv \lim _{N, M_{R}} M_{R}^{2} \sum_{l=1}^{N} c_{l}^{N}(l \ln l)=\lim _{N, M_{R}} M_{R}^{2} / \ln N
\end{aligned}
$$

By comparing the above results with the ones obtained by naive cutoff regularizaton, it is easily seen that the $\mu_{s}$ sets an IR 'cutoff' at $M^{2}=0$ and $M_{c}$ provides an UV 'cutoff'. For renormalizable quantum field theories, $M_{c}$ can be taken to be infinity $\left(M_{c} \rightarrow \infty\right)$. In a theory without infrared divergence, $\mu_{s}$ can safely run to $\mu_{s}=0$. Actually, in the case that $M_{c} \rightarrow \infty$ and $\mu_{s}=0$, one recovers the initial integral. Also once $M_{R}$ and $N$ are taken to be infinity, the regularized theory becomes independent of the regularization prescription. These are main properties needed for a proper regularization. For a detailed description and an explicit treatment for higher loop Feynman integrals, it is referred to the original paper on loop regularization [1, 2]. Note that to evaluate the ILIs, the algebraic computing for multi $\gamma$ matrices involving loop momentum $\not k$ such as $\not k \gamma_{\mu} \not k$ should be carried out to be expressed in terms of the independent components: $\gamma_{\mu}, \sigma_{\mu \nu}, \gamma_{5} \gamma_{\mu}, \gamma_{5}$.

It is known that in all the regularization schemes, there is an important issue that for a divergent integral it is in general not appropriate to shift the integration variable. In the loop regularization method we have actually shifted the integration variables before taking the regularization prescription, one may doubt wether such a treatment is well justified. The answer is yes. In fact, we can take the loop regularization prescription before shifting the integration variables, and the results are the same as what we get when shifting the integration variables first. For an illustration, let us examine a simple logarithmic divergent Feynman integral:

$$
L=\int \frac{d^{4} k}{(2 \pi)^{4}} \frac{1}{k^{2}-m_{1}^{2}} \frac{1}{(k-p)^{2}-m_{2}^{2}}
$$

Following the standard process of the loop regularization method, the first step is to apply the general Feynman parameter formula

$$
\begin{aligned}
\frac{1}{a_{1}^{\alpha_{1}} a_{2}^{\alpha_{2}} \cdots a_{n}^{\alpha_{n}}}= & \frac{\Gamma\left(\alpha_{1}+\cdots+\alpha_{n}\right)}{\Gamma\left(\alpha_{1}\right) \cdots \Gamma\left(\alpha_{n}\right)} \int_{0}^{1} d x_{1} \int_{0}^{x_{1}} d x_{2} \cdots \int_{0}^{x_{n-2}} d x_{n-1} \\
& \frac{\left(1-x_{1}\right)^{\alpha_{1}-1}\left(x_{1}-x_{2}\right)^{\alpha_{2}-1} \cdots x_{n-1}^{\alpha_{n}-1}}{\left[a_{1}\left(1-x_{1}\right)+a_{2}\left(x_{1}-x_{2}\right)+\cdots+a_{n} x_{n-1}\right]^{\alpha_{1}+\cdots+\alpha_{n}}}
\end{aligned}
$$

to the Feyman integral. For the above Feynman integral, we then obtain the following integral

$$
L=\int \frac{d^{4} k}{(2 \pi)^{4}} \int_{0}^{1} d x \frac{1}{\left\{(1-x)\left(k^{2}-m_{1}^{2}\right)+x\left[(k-p)^{2}-m_{2}^{2}\right]\right\}^{2}}
$$




$$
\begin{aligned}
& =\int \frac{d^{4} k}{(2 \pi)^{4}} \int_{0}^{1} d x \frac{1}{\left\{(k-x p)^{2}-\left[(1-x) m_{1}^{2}+x m_{2}^{2}-x(1-x) p^{2}\right]\right\}^{2}} \\
& =\int_{0}^{1} d x \int \frac{d^{4} k}{(2 \pi)^{4}} \frac{1}{\left((k-x p)^{2}-M^{2}\right)^{2}}
\end{aligned}
$$

with $M^{2}=(1-x) m_{1}^{2}+x m_{2}^{2}-x(1-x) p^{2}$. When shifting the integration variable, we arrive at the standard scalar type ILI

$$
L=\int_{0}^{1} d x \int \frac{d^{4} k}{(2 \pi)^{4}} \frac{1}{\left(k^{2}-M^{2}\right)^{2}}=\int_{0}^{1} d x I_{0}
$$

By making Wick rotation and applying the loop regularization prescription to such an integral, we then obtain the regularized Feynman integral

$$
L^{R}=i \int_{0}^{1} d x \lim _{N, M_{l}^{2}} \sum_{l=0}^{N} c_{l}^{N} \int \frac{d^{4} k}{(2 \pi)^{4}} \frac{1}{\left(k^{2}+M^{2}+M_{l}^{2}\right)^{2}}
$$

Alternatively, one can also apply for the regularization prescription before shifting the integration variable, i.e., $(k-x p)^{2} \rightarrow(k-x p)^{2}+M_{l}^{2}$, we then have

$$
L^{\prime R}=i \lim _{N, M_{l}^{2}} \sum_{l=0}^{N} c_{l}^{N} \int \frac{d^{4} k}{(2 \pi)^{4}} \frac{1}{\left[(k-x p)^{2}+M^{2}+M_{l}^{2}\right]^{2}}
$$

which becomes a well defined integral, so that we can safely shift the integration variable:

$$
L^{\prime R}=\int_{0}^{1} d x \lim _{N, M_{l}^{2}} \sum_{l=0}^{N} c_{l}^{N} \int \frac{d^{4} k}{(2 \pi)^{4}} \frac{1}{\left(k^{2}+M^{2}+M_{l}^{2}\right)^{2}} \equiv L^{R}
$$

which explicitly shown that in loop regularization method, one can safely shift the integration variables and express all the Feynman integrals in terms of ILIs before applying for the regularization prescription. In fact, it was found from the calculation of triangle anomaly that even for the linear divergent integral, only when firstly making a shift of integral variable, which then allows one to eliminate the ambiguities and leads to a consistent result. The reason is simple that loop regularization is translational invariant.

\section{CHECKING WARD-TAKAHASKI-SLAVNOV-TAYLOR IDENTITIES WITH EXPLICIT CALCULATIONS OF RENORMALIZATION CONSTANTS AND $\beta$ FUNCTION}

With the above analyzes, we are in the position to calculate the renormalization constants of Non-Abelian gauge theory at one loop level by using the loop regularization method. More details can be found in Appendix $\mathrm{C}$ where we evaluate all the one-loop divergent diagrams in terms of the explicit forms of ILIs.

\section{A. Renormalization constant for fermion fields strength}

As there is only one diagram which contributes the one-loop renormalization for the fermion fields strength, the divergent part of this diagram has been evaluated in detail in the Appendix $\mathrm{C}$ and explicitly given in terms of the ILIs. Here we only write down the regularized divergent part for the purpose of defining the relevant renormalization constant

$$
L(2)_{d i v}=\left(-g^{2} C_{2}\right) \int_{0}^{1} d x_{1}\left\{\left[x_{1}\left(3 x_{1}-4\right)(\xi-1)-2 x_{1}\right] p p+\left[2 x_{1}(\xi-1)+4\right] m\right\} I_{0}^{R}
$$

The explicit form of $I_{0}^{R}$ is given in loop regularization by the following form

$$
I_{0}^{R}=\frac{i}{16 \pi^{2}}\left[\ln \frac{M_{c}^{2}}{\mu^{2}}-\gamma_{\omega}+y_{0}\left(\frac{\mu^{2}}{M_{c}^{2}}\right)\right]
$$


The next step is to introduce appropriate renormalization conditions to make a suitable subtraction. Namely we shall find a prescription to divide the Feynman integral into divergent part and finite part, and cancel the divergent part by the counterterms. Such a prescription will fix the renormalization constants uniquely. Many different ways to introduce the renormalization conditions have been put forward in literature, they are referred as various renormalization schemes, such as: On-Shell renormalization scheme, Momentum Subtraction scheme, Minimal Subtraction scheme, and so on. Different renormalization schemes will lead to different definitions of the renormalized parameters. Nevertheless, the physics content of the theory, i.e. the renormalized S matrix elements, should not depend on the choices of renormalization schemes [28].

As is well-known, no matter under which renormalization schemes, it is inevitable to involve a mass dimensional parameter into the original theory, even though the original theory contains only dimensionless parameters. For example, in Momentum Subtraction scheme, one needs set the reference momentum point for subtraction, and in Minimal Subtraction scheme one has to introduce a mass dimensional parameter $\mu$. In fact, this is the essential reason of the dimension transmutation [29]. Any choice for the involved parameter is as good as any other, the physics should be invariant under the transformations which merely change this parameter. This is actually the consequence of renormalization group. Such a mass dimensional parameter plays the role of physically interesting sliding energy scale.

To remove the infinities, it needs to specify the subtraction scheme. In the loop regularization method, we may adopt, for simplicity, a subtraction scheme similar to the Modified Minimal Subtraction scheme in dimensional regularization. Notice that the arbitrary mass parameter $\mu_{s}$ plays the role of the sliding energy scale, one may rewrite $I_{0}^{R}$ as follows

$$
I_{0}^{R}=\frac{i}{16 \pi^{2}}\left[\ln \frac{M_{c}^{2}}{\mu_{s}^{2}}-\gamma_{\omega}\right]+\frac{i}{16 \pi^{2}}\left[\ln \frac{\mu_{s}^{2}}{\mu^{2}}+y_{0}\left(\frac{\mu^{2}}{M_{c}^{2}}\right)\right]
$$

Since the term $y_{0}$ approaches to zero $y_{0} \rightarrow 0$ in the limit $M_{c} \rightarrow \infty$. For the massless case with on mass shell condition, we have $\mu^{2}=\mu_{s}^{2}$ and $\ln \mu_{s}^{2} / \mu^{2}=0$. Thus the substraction scheme is chosen so that the terms proportional

to $\frac{i}{16 \pi^{2}}\left(\ln \frac{M_{c}^{2}}{\mu_{s}^{2}}-\gamma_{\omega}\right)$ in the Feynman integral are canceled by the introduction of counterterms. As such a term doesn't depend on the Feynman parameter $x_{1}$, one can integrate $x_{1}$ easily. Final results are given by:

$$
\begin{aligned}
L(2)_{d i v} & =\left(-g^{2} C_{2}\right) \int_{0}^{1} d x_{1}\left\{\left[x_{1}\left(3 x_{1}-4\right)(\xi-1)-2 x_{1}\right] \not p+\left[2 x_{1}(\xi-1)+4\right] m\right\} \frac{i}{16 \pi^{2}}\left(\ln \frac{M_{c}^{2}}{\mu_{s}^{2}}-\gamma_{\omega}\right) \\
& =\frac{-i g^{2}}{8 \pi^{2}} C_{2}[(-\xi) \not p+(\xi+3) m] \frac{1}{2}\left(\ln \frac{M_{c}^{2}}{\mu_{s}^{2}}-\gamma_{\omega}\right)
\end{aligned}
$$

From the condition $i\left(z_{2}-1\right) \not p+L(2)_{d i v}=0$, we then obtain the renormalization constant $z_{2}$ :

$$
z_{2}=1-\frac{g^{2}}{8 \pi^{2}} C_{2} \xi \frac{1}{2}\left(\ln \frac{M_{c}^{2}}{\mu_{s}^{2}}-\gamma_{\omega}\right)
$$

\section{B. Renormalization constant for gluon fields strength}

Four diagrams can contribute to the $A_{\mu}^{a}$ 's renormalization as shown in Fig.1. These four diagrams have explicitly been evaluated in [1, 2] with the result:

$$
\begin{aligned}
L_{R \mu \nu}^{a b}= & g^{2} \delta^{a b}\left(p^{2} g_{\mu \nu}-p_{\mu} p_{\nu}\right) \int_{0}^{1} d x\left\{C_{1}\left[1+4 x(1-x)+\frac{1}{2}(1-\xi)\right] I_{0}^{R}\right. \\
& \left.-N_{f} T_{2} 8 x(1-x) I_{0}^{R}(m)-4 C_{1}(1-\xi)\left[1-\frac{1}{8}(1-\xi)\right] x(1-x) p^{2} I_{-2}^{R}\right\}
\end{aligned}
$$

where $I_{0}^{R}$ is the renormalized divergent ILIs and given by Eq25] in the loop regularization. Thus the purely renormalized divergent term turns out to have the following form:

$$
\begin{aligned}
L_{\mu \nu ; d i v}^{a b}= & g^{2} \delta^{a b}\left(p^{2} g_{\mu \nu}-p_{\mu} p_{\nu}\right) \int_{0}^{1} d x\left\{C_{1}\left[1+4 x(1-x)+\frac{1}{2}(1-\xi)\right] \frac{i}{16 \pi^{2}}\left(\ln \frac{M_{c}^{2}}{\mu_{s}^{2}}-\gamma_{\omega}\right)\right. \\
& \left.-N_{f} T_{2} 8 x(1-x) \frac{i}{16 \pi^{2}}\left(\ln \frac{M_{c}^{2}}{\mu_{s}^{2}}-\gamma_{\omega}\right)\right\} \\
= & i\left\{\frac{g^{2}}{16 \pi^{2}}\left(\frac{13}{3}-\xi\right) C_{1} \frac{1}{2}\left(\ln \frac{M_{c}^{2}}{\mu_{s}^{2}}-\gamma_{\omega}\right)-\frac{g^{2}}{6 \pi^{2}} N_{f} T_{2} \frac{1}{2}\left(\ln \frac{M_{c}^{2}}{\mu_{s}^{2}}-\gamma_{\omega}\right)\right\} \delta^{a b}\left(p^{2} g_{\mu \nu}-p_{\mu} p_{\nu}\right)
\end{aligned}
$$


The above divergent term can be canceled by introducing the counterterm

$$
i\left(z_{3}-1\right) \delta^{a b}\left(p^{2} g_{\mu \nu}-p_{\mu} p_{\nu}\right)=L_{\mu \nu ; d i v}^{a b}
$$

with the renormalization constant $z_{3}$

$$
z_{3}=1+\frac{g^{2}}{16 \pi^{2}}\left[\left(\frac{13}{3}-\xi\right) C_{1}-\frac{g^{2}}{6 \pi^{2}} N_{f} T_{2}\right] \frac{1}{2}\left(\ln \frac{M_{c}^{2}}{\mu_{s}^{2}}-\gamma_{\omega}\right)
$$

\section{Ghost self-energy diagram and renormalization of ghost fields}

There is only one diagram (fig.3) which contributes to the one-loop renormalization for the ghost fields strength. The divergent part of this diagram is evaluated in the Appendix $\mathrm{C}$ and reads in terms of the renormalized divergent ILIs as follows

$$
L(3)_{d i v}^{c d}=-C_{1} g^{2} \delta^{c d} \int_{0}^{1} d x \int \frac{d^{4} k}{(2 \pi)^{4}}\left(x-\left(1-\frac{3}{2} x\right)(\xi-1)\right) p^{2} I_{0}^{R}
$$

Using Eq.(25) and noticing that the subtracting divergent term $\frac{i}{16 \pi^{2}} \ln \frac{M_{c}^{2}}{\mu_{s}^{2}}$ is independent of the Feynman parameter $x$, we have

$$
L(3)_{d i v}^{c d}=\frac{i g^{2}}{16 \pi^{2}}\left(\frac{1}{2} \xi-\frac{3}{2}\right) C_{1} \delta^{c d} p^{2} \frac{1}{2}\left(\ln \frac{M_{c}^{2}}{\mu_{s}^{2}}-\gamma_{\omega}\right)
$$

The counterterm should satisfy the condition

$$
i\left(\tilde{z}_{3}-1\right) p^{2} \delta^{c d}+L(3)_{d i v}^{c d}=0
$$

which leads the renormaliztion constant $\tilde{z_{3}}$ to be

$$
\tilde{z_{3}}=1+\frac{g^{2}}{16 \pi^{2}} C_{1}\left(\frac{3}{2}-\frac{\xi}{2}\right) \frac{1}{2}\left(\ln \frac{M_{c}^{2}}{\mu_{s}^{2}}-\gamma_{\omega}\right)
$$

\section{Fermion-gluon vertex renormalization}

Two kind of diagrams including their permutation (fig.4) contribute to the one-loop renormalization for the fermiongluon vertex. They are explicitly evaluated in the Appendix $\mathrm{C}$, the divergent parts are given in terms of the renormalized divergent ILIs as follows

$$
\begin{aligned}
L(4 a)_{\mu ; d i v}^{a R} & =g^{3}\left(C_{2}-\frac{1}{2} C_{1}\right) T^{a} \int_{0}^{1} d x_{1} \int_{0}^{x_{1}} d x_{2}\left[2+6\left(1-x_{1}\right)(\xi-1)\right] \gamma_{\mu} I_{0}^{R}\left(M_{4 a}\right) \\
L(4 b)_{\mu ; d i v}^{a R} & =g^{3} C_{1} T^{a} \gamma_{\mu} \int_{0}^{1} d x_{1} \int_{0}^{x_{1}} d x_{2}\left[3+\frac{9}{4} x_{1}(\xi-1)\right] I_{0}^{R}\left(M_{4 b}\right)
\end{aligned}
$$

The corresponding subtracting divergent terms are found to be

$$
\begin{aligned}
L(4 a)_{\mu ; d i v}^{a R} & =\frac{i g^{3}}{8 \pi^{2}}\left(C_{2}-\frac{1}{2} C_{1}\right) \xi \gamma_{\mu} T^{a} \frac{1}{2}\left(\ln \frac{M_{c}^{2}}{\mu_{s}^{2}}-\gamma_{\omega}\right) \\
L(4 b)_{\mu ; d i v}^{a R} & =\frac{i g^{3}}{8 \pi^{2}} \frac{3}{4}(\xi+1) C_{1} T^{a} \gamma_{\mu} \frac{1}{2}\left(\ln \frac{M_{c}^{2}}{\mu_{s}^{2}}-\gamma_{\omega}\right)
\end{aligned}
$$

The total contribution is given

$$
\begin{aligned}
L(4)_{\mu ; d i v}^{a R} & =L(4 a)_{\mu ; d i v}^{a R}+L(4 b)_{\mu ; d i v}^{a R} \\
& =\frac{i g^{3}}{8 \pi^{2}}\left[\left(\frac{3}{4}+\frac{1}{4} \xi\right) C_{1}+\xi C_{2}\right] T^{a} \gamma_{\mu} \frac{1}{2}\left(\ln \frac{M_{c}^{2}}{\mu_{s}^{2}}-\gamma_{\omega}\right)
\end{aligned}
$$

From the renormaliztion condition $\left(z_{1 F}-1\right) i g T^{a} \gamma_{\mu}+L(4)_{\mu ; d i v}^{a R}=0$, the renormalization constant $z_{1 F}$ reads:

$$
z_{1 F}=1-\frac{g^{2}}{8 \pi^{2}}\left[\left(\frac{3}{4}+\frac{\xi}{4}\right) C_{1}+\xi C_{2}\right] \frac{1}{2}\left(\ln \frac{M_{c}^{2}}{\mu_{s}^{2}}-\gamma_{\omega}\right)
$$




\section{E. Ghost-gluon vertex renormalization}

For the one-loop renormalization of three-gluon vertex, there are two diagrams including their permutation (fig.5). Their explicit evaluation is presented in the Appendix C. The divergent parts are given in terms of the renormalized divergent ILIs as follows

$$
\begin{aligned}
L(5 a)_{\mu ; d i v}^{a c b} & =-\frac{i}{2} g^{3} C_{1} f^{a c b} \int_{0}^{1} d x_{1} \int_{0}^{x_{1}} d x_{2} \frac{1}{2} \xi p_{2 \mu} I_{0}^{R}\left(M_{5 a}\right) \\
L(5 b)_{\mu ; d i v}^{a c b} & =-\frac{3 i}{4} g^{3} C_{1} f^{a c b} \int_{0}^{1} d x_{1} \int_{0}^{x_{1}} d x_{2}\left(3\left(x_{1}-x_{2}\right)(\xi-1)+1\right) p_{2 \mu} I_{0}^{R}\left(M_{5 b}\right)
\end{aligned}
$$

The corresponding subtracting divergent terms are given by integrating over Feynman parameters $x_{1}, x_{2}$

$$
\begin{aligned}
L(5 a)_{\mu ; d i v}^{a c b} & =\frac{g^{3}}{16 \pi^{2}} \frac{1}{4} \xi C_{1} f^{a c b} p_{2 \mu} \frac{1}{2}\left(\ln \frac{M_{c}^{2}}{\mu_{s}^{2}}-\gamma_{\omega}\right) \\
L(5 b)_{\mu ; d i v}^{a c b} & =\frac{g^{3}}{16 \pi^{2}} \frac{3}{4} \xi C_{1} f^{a c b} p_{2 \mu} \frac{1}{2}\left(\ln \frac{M_{c}^{2}}{\mu_{s}^{2}}-\gamma_{\omega}\right)
\end{aligned}
$$

with the final result

$$
\begin{aligned}
L(5)_{\mu ; d i v}^{a c b} & =L(5 a)_{\mu ; d i v}^{a c b}+L(5 b)_{\mu ; d i v}^{a c b} \\
& =\frac{g^{3}}{16 \pi^{2}} \xi C_{1} f^{a c b} p_{2 \mu} \frac{1}{2}\left(\ln \frac{M_{c}^{2}}{\mu_{s}^{2}}-\gamma_{\omega}\right)
\end{aligned}
$$

From the renormalization condition $\left(\tilde{z_{1}}-1\right) g f^{a c b} p_{2 \mu}+L(5)_{\mu ; d i v}^{a c b}=0$, the renormalization constant $\tilde{z_{1}}$ is given by:

$$
\tilde{z_{1}}=1-\frac{g^{2}}{16 \pi^{2}} \xi C_{1} \frac{1}{2}\left(\ln \frac{M_{c}^{2}}{\mu_{s}^{2}}-\gamma_{\omega}\right)
$$

\section{F. Three-gluon vertex renormalization}

Four loop diagrams including their permutation graphs will contribute to the one-loop renormalization of three-gluon vertex. More detailed evaluation is presented in the Appendix $\mathrm{C}$, the divergent parts in terms of the renormalized divergent ILIs read

$$
\begin{aligned}
L(6 a)_{\mu \nu \lambda ; d i v}^{a b c}= & 2 i g^{3} f^{a b c} T_{2} \int_{0}^{1} d x_{1} \int_{0}^{x_{1}} d x_{2} I_{0}^{R}\left(M_{6 a}\right)\left[4 ( - x _ { 1 } + x _ { 2 } + 1 ) \left(\frac{1}{2} k_{2 \mu} g_{\nu \lambda}+\right.\right. \\
& \left.+\frac{1}{2} k_{2 \nu} g_{\mu \lambda}+k_{2 \lambda} g_{\mu \nu}-k_{2 \mu} g_{\nu \lambda}-k_{2 \nu} g_{\mu \lambda}-\frac{1}{2} k_{2 \lambda} g_{\mu \nu}\right)+x_{2}\left(\frac{1}{2} k_{3 \mu} g_{\nu \lambda}+\right. \\
& \left.+\frac{1}{2} k_{3 \nu} g_{\mu \lambda}+k_{3 \lambda} g_{\mu \nu}-k_{3 \mu} g_{\nu \lambda}-k_{3 \nu} g_{\mu \lambda}-\frac{1}{2} k_{3 \lambda} g_{\mu \nu}\right)+ \\
& +\left(x_{2}-x_{1}\right)\left(\frac{1}{2} k_{2 \nu} g_{\lambda \mu}+\frac{1}{2} k_{2 \lambda} g_{\nu \mu}+k_{2 \mu} g_{\nu \lambda}-k_{2 \nu} g_{\lambda \mu}-k_{2 \lambda} g_{\nu \mu}-\frac{1}{2} k_{2 \mu} g_{\nu \lambda}\right)+ \\
& +x_{2}\left(\frac{1}{2} k_{3 \nu} g_{\lambda \mu}+\frac{1}{2} k_{3 \lambda} g_{\nu \mu}+k_{3 \mu} g_{\nu \lambda}-k_{3 \nu} g_{\lambda \mu}-k_{3 \lambda} g_{\nu \mu}-\frac{1}{2} k_{3 \mu} g_{\nu \lambda}\right)+ \\
& +\left(x_{2}-x_{1}\right)\left(\frac{1}{2} k_{2 \lambda} g_{\mu \nu}+\frac{1}{2} k_{2 \mu} g_{\lambda \nu}+k_{2 \nu} g_{\lambda \mu}-k_{2 \lambda} g_{\mu \nu}-k_{2 \mu} g_{\lambda \nu}-\frac{1}{2} k_{\nu} g_{\lambda \mu}\right)+ \\
& \left.+\left(x_{2}-1\right)\left(\frac{1}{2} k_{3 \lambda} g_{\mu \nu}+\frac{1}{2} k_{3 \mu} g_{\lambda \nu}+k_{3 \nu} g_{\lambda \mu}-k_{3 \lambda} g_{\mu \nu}-k_{3 \mu} g_{\lambda \nu}-\frac{1}{2} k_{3 \nu} g_{\lambda \mu}\right)\right] \\
L(6 b)_{\mu \nu \lambda ; d i v}^{a b c}= & 2 i g^{3} f^{a n m} f^{b m p} f^{c p n} \int_{0}^{1} d x_{1} \int_{0}^{x_{1}} d x_{2} I_{0}^{R}\left(M_{6 b}\right) \\
& \left\{\left[-\left(1-x_{1}\right) k_{2}+x_{2} k_{3}\right]_{\lambda} \frac{1}{4} g_{\mu \nu}+\left(x_{1} k_{2}+x_{2} k_{3}\right)_{\nu} \frac{1}{4} g_{\mu \lambda}+\left[-\left(1-x_{1}\right) k_{2}-\left(1-x_{2}\right) k_{3}\right]_{\mu} \frac{1}{4} g_{\nu \lambda}\right\} \\
& +\left(k_{2} \rightarrow k_{3}, \nu \rightarrow \lambda, b \rightarrow c\right)
\end{aligned}
$$




$$
\begin{aligned}
L(6 c)_{\mu \nu \lambda ; d i v}^{a b c}= & -i C_{1} g^{3} f^{a b c} \int_{0}^{1} d x_{1} \int_{0}^{x_{1}} d x_{2} I_{0}^{R}\left(M_{6 c}\right) \\
& \times\left\{\left(\frac{1}{4} g^{\alpha} \gamma g_{\lambda \nu}-\frac{2}{4} g_{\lambda}^{\alpha} g_{\nu \gamma}-\frac{1}{4} g_{\gamma \lambda} g_{\nu}^{\alpha}-\frac{1}{4} g_{\nu}^{\alpha} g_{\lambda \gamma}-\frac{2}{4} g_{\nu \gamma} g_{\lambda}^{\alpha}+\frac{4}{4} g_{\nu \lambda} g_{\gamma}^{\alpha}+g_{\nu}^{\alpha} g_{\gamma \lambda}\right)\right. \\
& \times\left[\left(\left(-1-x_{1}\right) k_{2}+\left(-1-x_{2}\right) k_{3}\right)^{\gamma} g_{\mu \alpha}+\left(\left(-1+2 x_{1}\right) k_{2}+\left(-1+2 x_{2}\right) k_{3}\right)_{\mu} g_{\alpha}^{\gamma}+\left(\left(2-x_{1}\right) k_{2}\right.\right. \\
& \left.\left.+\left(2-x_{2}\right) k_{3}\right)_{\alpha} g_{\mu}^{\gamma}\right]+\left(-\frac{2}{4} g_{\alpha \lambda} g_{\mu}^{\rho}-\frac{1}{4} g_{\alpha \mu} g_{\lambda}^{\rho}+\frac{4}{4} g_{\mu \lambda} g_{\alpha}^{\rho}+\frac{1}{4} g_{\alpha}^{\rho} g_{\mu \lambda}-\frac{1}{4} g_{\lambda}^{\rho} g_{\mu \alpha}-\frac{2}{4} g_{\mu}^{\rho} g_{\alpha \lambda}+g_{\lambda}^{\rho} g_{\alpha \mu}\right) \\
& \times\left[\left(\left(2-x_{1}\right) k_{2}-x_{2} k_{3}\right)^{\alpha} g_{\nu \rho}+\left(\left(-1+2 x_{1}\right) k_{2}+2 x_{2} k_{3}\right)_{\nu} g_{\rho}^{\alpha}+\left(\left(-1-x_{1}\right) k_{2}-x_{2} k_{3}\right)_{\rho} g_{\nu}^{\alpha}\right] \\
& +\left(-\frac{1}{4} g_{\mu}^{\gamma} g_{\nu \rho}+g_{\mu}^{\gamma} g_{\nu \rho}-\frac{2}{4} g_{\nu}^{\gamma} g \mu \rho+\frac{4}{4} g_{\mu \nu} g_{\rho}^{\gamma}+\frac{1}{4} g_{\rho}^{\gamma} g_{\mu \nu}-\frac{2}{4} g_{\mu \rho} g_{\nu}^{\gamma}-\frac{1}{4} g_{\nu \rho} g_{\mu}^{\gamma}\right) \\
& \times\left[\left(\left(1-x_{1}\right) k_{2}+\left(2-x_{2}\right) k_{3}\right)^{\rho} g_{\lambda \gamma}+\left(\left(-2+2 x_{1}\right) k_{2}+\left(-1+2 x_{2}\right) k_{3}\right)_{\lambda} g_{\gamma}^{\rho}\right. \\
& \left.\left.+\left(\left(1-x_{1}\right) k_{2}+\left(-1-x_{2}\right) k_{3}\right)_{\gamma} g_{\lambda}^{\rho}\right]\right\} \\
L(6 d)_{\mu \nu \lambda ; d i v}^{a b c}= & \frac{3 i}{4} g^{3} C_{1} f^{a b c}\left(g_{\nu \rho} g_{\lambda \sigma}-g_{\rho \lambda} g_{\sigma \nu}\right) \int_{0}^{1} d x_{1} I_{0}^{R}\left(M_{6 d}\right)\left[\left(1+x_{1}\right) k_{1}^{\sigma} g_{\mu}^{\rho}+\left(1-2 x_{1}\right) k_{1 \mu} g^{\rho \sigma}+\left(-2+x_{1}\right) k_{1}^{\rho} g_{\mu}^{\sigma}\right] \\
& + \text { permutation graphs }
\end{aligned}
$$

The corresponding subtracting divergent terms are simply obtained by integrating over the Feynman parameters $x_{1}$, $x_{2}$

$$
\begin{aligned}
L(6 a)_{\mu \nu \lambda ; d i v}^{a b c} & =-\frac{4}{3} i g^{2} T_{2} \frac{i}{16 \pi^{2}}\left(\ln \frac{M_{c}^{2}}{\mu_{s}^{2}}-\gamma_{\omega}\right) g f^{a b c}\left[g_{\mu \nu}\left(k_{1 \lambda}-k_{2 \lambda}\right)+g \nu \lambda\left(k_{2 \mu}-k_{3 \mu}\right)+g \lambda \mu\left(k_{3 \nu}-k_{1 \nu}\right)\right] \\
L(6 b)_{\mu \nu \lambda ; d i v}^{a b c} & =\frac{i}{24} g^{2} C_{1} \frac{i}{16 \pi^{2}}\left(\ln \frac{M_{c}^{2}}{\mu_{s}^{2}}-\gamma_{\omega}\right) g f^{a b c}\left[g_{\mu \nu}\left(k_{1}-k_{2}\right)_{\lambda}+g_{\nu \lambda}\left(k_{2}-k_{3}\right)_{\mu}+g_{\lambda \mu}\left(k_{3}-k_{1}\right)_{\nu}\right] \\
L(6 c)_{\mu \nu \lambda ; d i v}^{a b c} & =-\frac{13 i}{8} C_{1} g^{2} \frac{i}{16 \pi^{2}}\left(\ln \frac{M_{c}^{2}}{\mu_{s}^{2}}-\gamma_{\omega}\right) g f^{a b c}\left[g_{\mu \nu}\left(k_{1}-k_{2}\right)_{\lambda}+g_{\nu \lambda}\left(k_{2}-k_{3}\right)_{\mu}+g_{\lambda \mu}\left(k_{3}-k_{1}\right)_{\nu}\right] \\
L(6 d)_{\mu \nu \lambda ; d i v}^{a b c} & =\frac{9 i}{4} C_{1} g^{2} \frac{i}{16 \pi^{2}}\left(\ln \frac{M_{c}^{2}}{\mu_{s}^{2}}-\gamma_{\omega}\right) g f^{a b c}\left[g_{\mu \nu}\left(k_{1}-k_{2}\right)_{\lambda}+g_{\nu \lambda}\left(k_{2}-k_{3}\right)_{\mu}+g_{\lambda \mu}\left(k_{3}-k_{1}\right)_{\nu}\right]
\end{aligned}
$$

with the total result being given by summing over the four diagrams including their permutation graphs

$$
\begin{aligned}
L(6)_{\mu \nu \lambda ; d i v}^{a b c} & =N_{f} L(6 a)_{\mu \nu \lambda ; d i v}^{a b c}+L(6 b)_{\mu \nu \lambda ; d i v}^{a b c}+L(6 c)_{\mu \nu \lambda ; d i v}^{a b c}+L(6 d)_{\mu \nu \lambda ; d i v}^{a b c} \\
& =\left[\left(\frac{2}{3} i g^{2} C_{1}-\frac{4}{3} i g^{2} N_{f} T_{2}\right) \frac{i}{16 \pi^{2}}\left(\ln \frac{M_{c}^{2}}{\mu_{s}^{2}}-\gamma_{\omega}\right)\right] g f^{a b c}\left[g_{\mu \nu}\left(k_{1 \lambda}-k_{2 \lambda}\right)+g_{\nu \lambda}\left(k_{2 \mu}-k_{3 \mu}\right)+g_{\lambda \mu}\left(k_{3 \nu}-k_{1 \nu}\right)\right]
\end{aligned}
$$

Using the renormalization condition

$$
\left(z_{1}-1\right) g f^{a b c}\left[g_{\mu \nu}\left(k_{1 \lambda}-k_{2 \lambda}\right)+g_{\nu \lambda}\left(k_{2 \mu}-k_{3 \mu}\right)+g_{\lambda \mu}\left(k_{3 \nu}-k_{1 \nu}\right)\right]+L(6)_{\mu \nu \lambda ; d i v}^{a b c}=0
$$

we obtain the renormalization constant $z_{1}$ in Feynman gauge $\xi=1$ to be

$$
z_{1}=1+\left(\frac{g^{2}}{12 \pi^{2}} C_{1}-\frac{g^{2}}{6 \pi^{2}} N_{f} T_{2}\right) \frac{1}{2}\left(\ln \frac{M_{c}^{2}}{\mu_{s}^{2}}-\gamma_{\omega}\right)
$$

The evaluation in the $\xi$ gauge is rather length, the result is

$$
z_{1}=1+\left[\frac{g^{2}}{12 \pi^{2}}\left[1+\frac{9}{8}(1-\xi)\right] C_{1}-\frac{g^{2}}{6 \pi^{2}} N_{f} T_{2}\right] \frac{1}{2}\left(\ln \frac{M_{c}^{2}}{\mu_{s}^{2}}-\gamma_{\omega}\right)
$$

\section{G. Four-gluon vertex renormalization}

We finally consider the four-gluon vertex renormalization, there are five loop diagrams which contribute to its renormalizaion. The detailed evaluation can be found in the Appendix C, we present here only the divergent parts in 
terms of the renormalized divergent ILIs

$$
\begin{aligned}
& L(7 a)_{\mu \nu \lambda \rho ; d i v}^{a b c d}=6 g^{4} f^{a e f} f^{b f j} f^{c j n} f^{d n e} \int_{0}^{1} d x_{1} \int_{0}^{x_{1}} d x_{2} \int_{0}^{x_{2}} d x_{3}\left[\frac{5}{2}\left(g_{\mu \nu} g_{\lambda \rho}+g_{\mu \rho} g_{\nu \lambda}\right)+\right. \\
& \left.\frac{34}{24}\left(g_{\mu \nu} g_{\lambda \rho}+g_{\mu \lambda} g_{\nu \rho}+g_{\mu \rho} g_{\nu \lambda}\right)\right] I_{0}^{R}\left(M_{7 a}\right)+2 \text { permutations } \\
& L(7 b)_{\mu \nu \lambda \rho ; d i v}^{a b c d}=2 g^{4} f^{a e f} f^{d m e}\left[f^{l f b} f^{l c m}\left(g_{\lambda}^{\beta} g_{\nu}^{\chi}-g^{\beta \chi} g_{\nu \lambda}\right)+f^{l f c} f^{l m b}\left(g^{\beta \chi} g_{\lambda \nu}-g_{\nu}^{\beta} g_{\lambda}^{\chi}\right)+f^{l f m} f^{l b c}\left(g_{\nu}^{\beta} g_{\lambda}^{\chi}-g_{\lambda}^{\beta} g_{\nu}^{\chi}\right)\right] \\
& \int_{0}^{1} \int_{0}^{x_{1}} d x_{1} d x_{2}\left(g_{\beta \mu} g_{\rho \chi}-\frac{1}{4} g_{\beta \mu} g_{\rho \chi}-\frac{2}{4} g_{\beta \rho} g_{\mu \chi}+\frac{4}{4} g_{\mu \rho} g_{\beta \chi}+\frac{1}{4} g_{\beta \chi} g_{\mu \rho}-\frac{2}{4} g_{\mu \chi} g_{\beta \rho}-\frac{1}{4} g_{\rho \chi} g_{\mu \beta}\right) I_{0}^{R}\left(M_{7 b}\right) \\
& +5 \text { permutations } \\
& L(7 c)_{\mu \nu \lambda \rho ; d i v}^{a b c d}=\frac{1}{2} g^{4}\left[f^{e a i} f^{e j d}\left(g_{\mu \beta} g_{\alpha \rho}-g_{\mu \rho} g_{\alpha \beta}\right)+f^{e a j} f^{e d i}\left(g_{\mu \rho} g_{\beta \alpha}-g_{\mu \alpha} g_{\beta \rho}\right)+f^{e a d} f^{e i j}\left(g_{\mu \alpha} g_{\rho \beta}-g_{\mu \beta} g_{\rho \alpha}\right)\right] \times \\
& {\left[f^{f i b} f^{f c j}\left(g_{\lambda}^{\alpha} g_{\nu}^{\beta}-g^{\alpha \beta} g_{\nu \lambda}\right)+f^{f i c} f^{f j b}\left(g^{\alpha \beta} g_{\lambda \nu}-g_{\nu}^{\alpha} g_{\lambda}^{\beta}\right)+f^{f i j} f^{f b c}\left(g_{\nu}^{\alpha} g_{\lambda}^{\beta}-g_{\lambda}^{\alpha} g_{\nu}^{\beta}\right)\right]} \\
& \int_{0}^{1} d x_{1} I_{0}^{R}\left(M_{7 c}\right)+2 \text { permutations } \\
& L(7 d)_{\mu \nu \lambda \rho ; d i v}^{a b c d}=-\frac{1}{4} g^{4} f^{a i e} f^{b m i} f^{c p m} f^{d e p}\left(g_{\mu \nu} g_{\lambda \rho}+g_{\mu \lambda} g_{\nu \rho}+g_{\mu \rho} g_{\nu \lambda}\right) \int_{0}^{1} d x_{1} \int_{0}^{x_{1}} d x_{2} \int_{0}^{x_{2}} d x_{3} I_{0}^{R}\left(M_{7 d}\right)+ \\
& +5 \text { permutations } \\
& L(7 e)_{\mu \nu \lambda \rho ; d i v}^{a b c d}=-8 g^{4} \operatorname{Tr}\left(T^{a} T^{d} T^{c} T^{b}\right)\left(g_{\mu \nu} g_{\lambda \rho}-2 g_{\mu \lambda} g_{\nu \rho}+g_{\mu \rho} g_{\nu \lambda}\right) \int_{0}^{1} d x_{1} \int_{0}^{x_{1}} d x_{2} \int_{0}^{x_{2}} d x_{3} I_{0}^{R}\left(M_{7 e}\right)+ \\
& 5 \text { permutations }
\end{aligned}
$$

The corresponding subtracting divergent terms are yielded by integrating over the Feynman parameters $x_{1}, x_{2}, x_{3}$

$$
\begin{aligned}
& L(7 a)_{\mu \nu \lambda \rho ; d i v}^{a b c d}=g^{4} \frac{i}{16 \pi^{2}}\left(\ln \frac{M_{c}^{2}}{\mu_{s}^{2}}-\gamma_{\omega}\right)\left[g_{\mu \nu} g_{\lambda \rho}\left(\frac{47}{12} F^{a b c d}+\frac{17}{12} F^{a c b d}+\frac{47}{12} F^{a b d c}\right)+\right. \\
& \left.g_{\mu \lambda} g_{\nu \rho}\left(\frac{17}{12} F^{a b c d}+\frac{47}{12} F^{a c b d}+\frac{47}{12} F^{a b d c}\right)+g_{\mu \rho} g_{\nu \lambda}\left(\frac{47}{12} F^{a b c d}+\frac{47}{12} F^{a c b d}+\frac{17}{12} F^{a b d c}\right)\right] \\
& L(7 b)_{\mu \nu \lambda \rho ; d i v}^{a b c d}=g^{4} \frac{i}{16 \pi^{2}}\left(\ln \frac{M_{c}^{2}}{\mu_{s}^{2}}-\gamma_{\omega}\right)\left[g_{\mu \nu} g_{\lambda \rho}\left(-\frac{17}{2} F^{a b c d}+2 F^{a c b d}-\frac{17}{2} F^{a b d c}-\frac{3}{2} C_{1} f^{l a d} f^{l b c}-\frac{3}{2} C_{1} f^{l a c} f^{l b d}\right)+\right. \\
& g_{\mu \lambda} g_{\nu \rho}\left(2 F^{a b c d}-\frac{17}{2} F^{a c b d}-\frac{17}{2} F^{a b d c}+\frac{3}{2} C_{1} f^{l a d} f^{l b c}-\frac{3}{2} C_{1} f^{l a b} f^{l c d}\right)+ \\
& \left.g_{\mu \rho} g_{\nu \lambda}\left(-\frac{17}{2} F^{a b c d}-\frac{17}{2} F^{a c b d}+2 F^{a b d c}+\frac{3}{2} C_{1} f^{l a c} f^{l b d}+\frac{3}{2} C_{1} f^{l a b} f^{l c d}\right)\right] \\
& L(7 c)_{\mu \nu \lambda \rho ; d i v}^{a b c d}=g^{4} \frac{i}{16 \pi^{2}}\left(\ln \frac{M_{c}^{2}}{\mu_{s}^{2}}-\gamma_{\omega}\right)\left[g_{\mu \nu} g_{\lambda \rho}\left(2 C_{1} f^{e a d} f^{e b c}+2 C_{1} f^{e a c} f^{e b d}+3 F^{a b c d}+3 F^{a b d c}\right)+\right. \\
& g_{\mu \lambda} g_{\nu \rho}\left(2 C_{1} f^{e a b} f^{e c d}-2 C_{1} f^{e a d} f^{e b c}+3 F^{a b d c}+3 F^{a c b d}\right)+ \\
& g_{\mu \rho} g_{\nu \lambda}\left(-2 C_{1} f^{e a b} f^{e c d}-2 C_{1} f^{e a c} f^{e b d}+3 F^{a b c d}+3 F^{a c b d}\right) \\
& L(7 d)_{\mu \nu \lambda \rho ; d i v}^{a b c d}=g^{4} \frac{i}{16 \pi^{2}}\left(\ln \frac{M_{c}^{2}}{\mu_{s}^{2}}-\gamma_{\omega}\right)\left[g_{\mu \nu} g_{\lambda \rho}\left(-\frac{1}{12} F^{a b c d}-\frac{1}{12} F^{a c b d}-\frac{1}{12} F^{a b d c}\right)+\right. \\
& \left.g_{\mu \lambda} g_{\nu \rho}\left(-\frac{1}{12} F^{a b c d}-\frac{1}{12} F^{a c b d}-\frac{1}{12} F^{a b d c}\right)+g_{\mu \rho} g_{\nu \lambda}\left(-\frac{1}{12} F^{a b c d}-\frac{1}{12} F^{a c b d}-\frac{1}{12} F^{a b d c}\right)\right] \\
& L(7 e)_{\mu \nu \lambda \rho ; d i v}^{a b c d}=-\frac{4}{3} T_{2} g^{4} \frac{i}{16 \pi^{2}}\left(\ln \frac{M_{c}^{2}}{\mu_{s}^{2}}-\gamma_{\omega}\right) \\
& \left\{g_{\mu \nu} g_{\lambda \rho}\left(f^{a d l} f^{b c l}+f^{a c l} f^{b d l}\right)+g_{\mu \lambda} g_{\nu \rho}\left(f^{a b l} f^{c d l}-f^{a d l} f^{b c l}\right)+g_{\mu \rho} g_{\nu \lambda}\left(-f^{a b l} f^{c d l}-f^{a c l} f^{b d l}\right)\right\}
\end{aligned}
$$

with $F^{a b c d} \equiv f^{a e f} f^{b f g} f^{c g h} f^{d h e}$. By adding those five diagrams together, we have

$$
\begin{aligned}
L(7)_{\mu \nu \lambda \rho ; d i v}^{a b c d} & =L(7 a)_{\mu \nu \lambda \rho ; d i v}^{a b c d}+L(7 b)_{\mu \nu \lambda \rho ; d i v}^{a b c d}+L(7 c)_{\mu \nu \lambda \rho ; d i v}^{a b c d}+L(7 d)_{\mu \nu \lambda \rho ; d i v}^{a b c d}+N_{f} L(7 e)_{\mu \nu \lambda \rho ; d i v}^{a b c d} \\
& =g^{4} \frac{i}{16 \pi^{2}}\left(\ln \frac{M_{c}^{2}}{\mu_{s}^{2}}-\gamma_{\omega}\right)\left[g_{\mu \nu} g_{\lambda \rho}\left(\frac{47}{12} F^{a b c d}+\frac{17}{12} F^{a c b d}+\frac{47}{12} F^{a b d c}\right)+\right.
\end{aligned}
$$




$$
\begin{aligned}
& \left.g_{\mu \lambda} g_{\nu \rho}\left(\frac{17}{12} F^{a b c d}+\frac{47}{12} F^{a c b d}+\frac{47}{12} F^{a b d c}\right)+g_{\mu \rho} g_{\nu \lambda}\left(\frac{47}{12} F^{a b c d}+\frac{47}{12} F^{a c b d}+\frac{17}{12} F^{a b d c}\right)\right] \\
& +g^{4} \frac{i}{16 \pi^{2}}\left(\ln \frac{M_{c}^{2}}{\mu_{s}^{2}}-\gamma_{\omega}\right)\left[g_{\mu \nu} g_{\lambda \rho}\left(-\frac{17}{2} F^{a b c d}+2 F^{a c b d}-\frac{17}{2} F^{a b d c}-\frac{3}{2} C_{1} f^{l a d} f^{l b c}-\frac{3}{2} C_{1} f^{l a c} f^{l b d}\right)+\right. \\
& g_{\mu \lambda} g_{\nu \rho}\left(2 F^{a b c d}-\frac{17}{2} F^{a c b d}-\frac{17}{2} F^{a b d c}+\frac{3}{2} C_{1} f^{l a d} f^{l b c}-\frac{3}{2} C_{1} f^{l a b} f^{l c d}\right)+ \\
& \left.g_{\mu \rho} g_{\nu \lambda}\left(-\frac{17}{2} F^{a b c d}-\frac{17}{2} F^{a c b d}+2 F^{a b d c}+\frac{3}{2} C_{1} f^{l a c} f^{l b d}+\frac{3}{2} C_{1} f^{l a b} f^{l c d}\right)\right] \\
& +g^{4} \frac{i}{16 \pi^{2}}\left(\ln \frac{M_{c}^{2}}{\mu_{s}^{2}}-\gamma_{\omega}\right)\left[g_{\mu \nu} g_{\lambda \rho}\left(2 C_{1} f^{e a d} f^{e b c}+2 C_{1} f^{e a c} f^{e b d}+3 F^{a b c d}+3 F^{a b d c}\right)+\right. \\
& g_{\mu \lambda} g_{\nu \rho}\left(2 C_{1} f^{e a b} f^{e c d}-2 C_{1} f^{e a d} f^{e b c}+3 F^{a b d c}+3 F^{a c b d}\right)+ \\
& g_{\mu \rho} g_{\nu \lambda}\left(-2 C_{1} f^{e a b} f^{e c d}-2 C_{1} f^{e a c} f^{e b d}+3 F^{a b c d}+3 F^{a c b d}\right) \\
& +g^{4} \frac{i}{16 \pi^{2}}\left(\ln \frac{M_{c}^{2}}{\mu_{s}^{2}}-\gamma_{\omega}\right)\left[g_{\mu \nu} g_{\lambda \rho}\left(-\frac{1}{12} F^{a b c d}-\frac{1}{12} F^{a c b d}-\frac{1}{12} F^{a b d c}\right)+\right. \\
& \left.g_{\mu \lambda} g_{\nu \rho}\left(-\frac{1}{12} F^{a b c d}-\frac{1}{12} F^{a c b d}-\frac{1}{12} F^{a b d c}\right)+g_{\mu \rho} g_{\nu \lambda}\left(-\frac{1}{12} F^{a b c d}-\frac{1}{12} F^{a c b d}-\frac{1}{12} F^{a b d c}\right)\right] \\
& -\frac{4}{3} N_{f} T_{2} g^{4} \frac{i}{16 \pi^{2}}\left(\ln \frac{M_{c}^{2}}{\mu_{s}^{2}}-\gamma_{\omega}\right) \\
& \left\{g_{\mu \nu} g_{\lambda \rho}\left(f^{a d l} f^{b c l}+f^{a c l} f^{b d l}\right)+g_{\mu \lambda} g_{\nu \rho}\left(f^{a b l} f^{c d l}-f^{a d l} f^{b c l}\right)+g_{\mu \rho} g_{\nu \lambda}\left(-f^{a b l} f^{c d l}-f^{a c l} f^{b d l}\right)\right\} \\
& =g^{4} \frac{i}{16 \pi^{2}}\left(\ln \frac{M_{c}^{2}}{\mu_{s}^{2}}-\gamma_{\omega}\right)\left[g_{\mu \nu} g_{\lambda \rho}\left(\frac{1}{2} C_{1} f^{e a d} f^{e b c}+\frac{1}{2} C_{1} f^{e a c} f^{e b d}+\frac{-5}{3} F^{a b c d}+\frac{10}{3} F^{a c b d}+\frac{-5}{3} F^{a b d c}\right)+\right. \\
& g_{\mu \lambda} g_{\nu \rho}\left(\frac{1}{2} C_{1} f^{e a b} f^{e c d}-\frac{1}{2} C_{1} f^{e a d} f^{e b c}+\frac{-5}{3} F^{a b c d}+\frac{10}{3} F^{a c b d}+\frac{-5}{3} F^{a b d c}\right)+ \\
& \left.g_{\mu \rho} g_{\nu \lambda}\left(-\frac{1}{2} C_{1} f^{e a b} f^{e c d}-\frac{1}{2} C_{1} f^{e a c} f^{e b d}+\frac{-5}{3} F^{a b c d}+\frac{10}{3} F^{a c b d}+\frac{-5}{3} F^{a b d c}\right)\right] \\
& -\frac{4}{3} N_{f} T_{2} g^{4} \frac{i}{16 \pi^{2}}\left(\ln \frac{M_{c}^{2}}{\mu_{s}^{2}}-\gamma_{\omega}\right) \\
& \left\{g_{\mu \nu} g_{\lambda \rho}\left(f^{a d l} f^{b c l}+f^{a c l} f^{b d l}\right)+g_{\mu \lambda} g_{\nu \rho}\left(f^{a b l} f^{c d l}-f^{a d l} f^{b c l}\right)+g_{\mu \rho} g_{\nu \lambda}\left(-f^{a b l} f^{c d l}-f^{a c l} f^{b d l}\right)\right\} \\
& =\left[-\frac{1}{3} C_{1}-\frac{4}{3} N_{f} T_{2}\right] g^{4} \frac{i}{16 \pi^{2}}\left(\ln \frac{M_{c}^{2}}{\mu_{s}^{2}}-\gamma_{\omega}\right) \\
& \left\{g_{\mu \nu} g_{\lambda \rho}\left(f^{a d l} f^{b c l}+f^{a c l} f^{b d l}\right)+g_{\mu \lambda} g_{\nu \rho}\left(f^{a b l} f^{c d l}-f^{a d l} f^{b c l}\right)+g_{\mu \rho} g_{\nu \lambda}\left(-f^{a b l} f^{c d l}-f^{a c l} f^{b d l}\right)\right\}
\end{aligned}
$$

By applying for the renormalization condition

$$
\begin{aligned}
& \left(z_{4}-1\right)\left(-i g^{2}\right)\left\{g_{\mu \nu} g_{\lambda \rho}\left(f^{a d l} f^{b c l}+f^{a c l} f^{b d l}\right)+g_{\mu \lambda} g_{\nu \rho}\left(f^{a b l} f^{c d l}-f^{a d l} f^{b c l}\right)+g_{\mu \rho} g_{\nu \lambda}\left(-f^{a b l} f^{c d l}-f^{a c l} f^{b d l}\right)\right\} \\
& +L(7)_{\mu \nu \lambda ; d i v}^{a b c}=0
\end{aligned}
$$

we then obtain in the Feyman gauge $\xi=1$ the renormalization constant $z_{4}$

$$
z_{4}=1-\left(\frac{g^{2}}{24 \pi^{2}} C_{1}+\frac{g^{2}}{6 \pi^{2}} N_{f} T_{2}\right) \frac{1}{2}\left(\ln \frac{M_{c}^{2}}{\mu_{s}^{2}}-\gamma_{\omega}\right)
$$

A similar but length evaluation in the $\xi$ gauge leads to the result

$$
z_{4}=1-\left[\frac{g^{2}}{24 \pi^{2}}(1+3(\xi-1)) C_{1}+\frac{g^{2}}{6 \pi^{2}} N_{f} T_{2}\right] \frac{1}{2}\left(\ln \frac{M_{c}^{2}}{\mu_{s}^{2}}-\gamma_{\omega}\right)
$$

\section{H. Ward-Takahaski-Slavnov-Taylor identities and $\beta$ function}

We shall summarize all the renormalization constants in this section to check Ward-Takahaski-Slavnov-Taylor identities and calculate $\beta$ function. All the results are listed as below:

$$
z_{2}=1-\frac{g^{2}}{8 \pi^{2}} C_{2} \xi \frac{1}{2}\left(\ln \frac{M_{c}^{2}}{\mu_{s}^{2}}-\gamma_{\omega}\right)
$$




$$
\begin{aligned}
z_{3} & =1+\left[\frac{g^{2}}{16 \pi^{2}}\left(\frac{13}{3}-\xi\right) C_{1}-\frac{g^{2}}{6 \pi^{2}} N_{f} T_{2}\right] \frac{1}{2}\left(\ln \frac{M_{c}^{2}}{\mu_{s}^{2}}-\gamma_{\omega}\right) \\
\tilde{z_{3}} & =1+\frac{g^{2}}{16 \pi^{2}} C_{1}\left(\frac{3}{2}-\frac{\xi}{2}\right) \frac{1}{2}\left(\ln \frac{M_{c}^{2}}{\mu_{s}^{2}}-\gamma_{\omega}\right) \\
z_{1 F} & =1-\frac{g^{2}}{8 \pi^{2}}\left[\left(\frac{3}{4}+\frac{\xi}{4}\right) C_{1}+\xi C_{2}\right] \frac{1}{2}\left(\ln \frac{M_{c}^{2}}{\mu_{s}^{2}}-\gamma_{\omega}\right) \\
\tilde{z_{1}} & =1-\frac{g^{2}}{16 \pi^{2}} \xi C_{1} \frac{1}{2}\left(\ln \frac{M_{c}^{2}}{\mu_{s}^{2}}-\gamma_{\omega}\right) \\
z_{1} & =1+\left[\frac{g^{2}}{12 \pi^{2}}\left(\frac{17}{8}-\frac{9}{8} \xi\right) C_{1}-\frac{g^{2}}{6 \pi^{2}} N_{f} T_{2}\right] \frac{1}{2}\left(\ln \frac{M_{c}^{2}}{\mu_{s}^{2}}-\gamma_{\omega}\right) \\
z_{4} & =1-\left[\frac{g^{2}}{24 \pi^{2}}(-2+3 \xi) C_{1}+\frac{g^{2}}{6 \pi^{2}} N_{f} T_{2}\right] \frac{1}{2}\left(\ln \frac{M_{c}^{2}}{\mu_{s}^{2}}-\gamma_{\omega}\right)
\end{aligned}
$$

It is straight forward to verify explicitly the Ward-Takahaski-Slavnov-Taylor identities:

$$
z_{g}=\frac{z_{1 F}}{z_{3}^{1 / 2} z_{2}}=\frac{\tilde{z_{1}}}{z_{3}^{1 / 2} \tilde{z}_{3}}=\frac{z_{1}}{z_{3}^{3 / 2}}=\frac{z_{4}^{1 / 2}}{z_{3}}
$$

which leads to the gauge independent renormalization constant for the gauge coupling constant $g=z_{g}^{-1} g_{0}$

$$
z_{g}=1-\left(\frac{11}{48 \pi^{2}} C_{1}-\frac{1}{12 \pi^{2}} N_{f} T_{2}\right) g^{2} \frac{1}{2}\left(\ln \frac{M_{c}^{2}}{\mu_{s}^{2}}-\gamma_{\omega}\right)
$$

In the loop regularization method, the energy scale $\mu_{s}$ plays the role of the sliding energy scale. According to the definition of $\beta$ function, we obtain the one-loop $\beta$ function:

$$
\begin{aligned}
\beta(g) & \left.\triangleq \lim _{M_{c} \rightarrow \infty} \mu_{s} \frac{\partial}{\partial \mu_{s}} g\right|_{g_{0}, m_{0}} \\
& =\left.\lim _{M_{c} \rightarrow \infty} g \mu_{s} \frac{\partial}{\partial \mu_{s}} \ln z_{g}\right|_{g_{0}, m_{0}} \\
& \simeq g \mu_{s} \frac{\partial}{\partial \mu_{s}}\left[\left(\frac{11}{48 \pi^{2}} C_{1}-\frac{1}{12 \pi^{2}} N_{f} T_{2}\right) g^{2} \frac{1}{2}\left(\ln \frac{M_{c}^{2}}{\mu_{s}^{2}}-\gamma_{\omega}\right)\right] \\
& \simeq g^{3} \mu_{s}\left(\frac{11}{48 \pi^{2}} C_{1}-\frac{1}{12 \pi^{2}} N_{f} T_{2}\right) \frac{-1}{\mu_{s}} \\
& =-\frac{g^{3}}{(4 \pi)^{2}}\left(\frac{11}{3 \pi^{2}} C_{1}-\frac{4}{3 \pi^{2}} N_{f} T_{2}\right)
\end{aligned}
$$

which agrees with the well-known result obtained by using dimesional regularization. It is noticed that a simple corresponding for the logarithmic divergences between the loop regularization method and dimensional regularization scheme is

$$
\frac{2}{\varepsilon} \longleftrightarrow \ln \frac{M_{c}^{2}}{\mu_{s}^{2}}
$$

with $\varepsilon \rightarrow 0$ and $M_{c} \rightarrow \infty$.

\section{CONCLUSION}

We have performed a complete calculation for all one loop diagrams of non-Abelian gauge theory by using the loop regularization method [1, 2] and provided an explicit check for the consistency of loop regularization method from the Ward-Takahaski-Slavnov-Taylor identities satisfied among the renormalization constants. It has been shown that the loop regularization method can lead to a consistent $\beta$ function.

From above explicit calculations, the conclusions stated in [1, 2] become manifest that the loop regularization method preserves not only non-Abelian gauge symmetry, but also Lorentz and translational symmetries though the existence of two energy scales $M_{c}$ and $\mu_{s}$ introduced intrinsically in this method. As the scales $M_{c}$ and $\mu_{s}$ play 
the role of ultraviolet divergent cutoff and infrared divergent cutoff respectively, the loop regularization method can deal with both the ultraviolet and infrared divergences. The existence of two energy scales also makes the loop regularization to maintain the divergent behavior of original theories, while the quadratic divergences in gauge theories are found to cancel each other as the loop regularization preserves gauge symmetry. Thus both loop regularization and dimensional regularization lead to the same renormalization constants for gauge theories with making a simple replacement between $\ln M_{c} / \mu_{s}$ and $1 / \varepsilon$. The possible distinguishable properties between loop regularization and dimensional regularization may occur for treating chiral field theories with anomaly action concerning the $\gamma_{5}$ matrix [16, 17], and for deriving effective field theories with dynamically generated spontaneous symmetry breaking [15] as well as for applying to supersymmetric theories involving the exact dimension [32]. Finally, we would like to point out that the renormalization scheme dependence is not involved in our present consideration as our computation for the renormalization constants is only at the one loop level and our focus in this note is mainly on the check of Ward-Takahaski-Slavnov-Taylor identities among the renormalization constants. It is interesting to see that the loop regularization method generally allows one to make on-shell renormalization prescription due to the existence of the energy scale $\mu_{s}$ which plays the role of infrared cutoff and sliding energy scale, such a feature may provide a practical way for reducing the renormalization scheme dependence, which is worthwhile to be further investigated elsewhere.

\section{Acknowledgments}

The authors would like to thank Einhorn Marty for valuable discussions during the KITPC program. This work was supported in part by the National Science Foundation of China (NSFC) under the grant 10475105, 10491306 and the Project of Knowledge Innovation Program (PKIP) of Chinese Academy of Sciences.

\section{APPENDIX A: SOME USEFUL FORMULAE OF COMPACT SIMPLE LIE GROUP}

In this appendix, we shall present some useful formulae about the structure constants and the traces of the representation matrices for Lie group.

For compact simple Lie group, one can choose the killing form of group to be in proportion to a unit matrix, then the Lie algebras satisfy the following identities:

$$
\begin{aligned}
& \operatorname{Tr}\left(T^{a} T^{b}\right)=T_{2} \delta^{a b} ; \quad\left[T^{a}, T^{b}\right]=i f^{a b c} T^{c} ; \\
& f^{a b d} f^{d c e}+f^{b c d} f^{d a e}+f^{c a d} f^{d b e}=0 ; \\
& f^{a b c} f^{d b c}=C_{1} \delta^{a d} ; \quad T^{a} T^{a}=C_{2} I
\end{aligned}
$$

where $f^{a b c}=-i T_{2}^{-1} \operatorname{tr}\left(T^{a} T^{b} T^{c}-T^{b} T^{a} T^{c}\right)$ is completely antisymmetric, and $T^{a}$ are group generators in fundamental representation. Using the above relations, one can easily prove the following relations:

$$
\begin{gathered}
f^{a n m} f^{b m p} f^{c p n}=\frac{1}{2} C_{1} f^{a b c}, \quad T^{a} T^{b} T^{a}=\left(C_{2}-\frac{1}{2} C_{1}\right) T^{b} \\
T^{a b c d}+T^{a b d c}+T^{a c d b}+T^{a d c b}-2 T^{a c b d}-2 T^{a d b c}=T_{2}\left(f^{a d l} f^{b c l}+f^{a c l} f^{b d l}\right) \\
F^{a b c d}-2 F^{a c b d}+F^{a b d c}=\frac{1}{2} C_{1}\left(f^{a d l} f^{b c l}+f^{a c l} f^{b d l}\right)
\end{gathered}
$$

where $T^{a b c d} \equiv \operatorname{Tr}\left(T^{a} T^{b} T^{c} T^{d}\right)$ with $T^{a b c d}=T^{c y c l e}$, and $F^{a b c d} \equiv f^{a e f} f^{b f g} f^{c g h} f^{\text {dhe }}$ with $F^{a b c d}=F^{c y c l e}=F^{\text {inverse }}$

\section{APPENDIX B: FEYNMAN RULES FOR GAUGE THEORY}

$$
\mathcal{L}=\bar{\psi}_{n}\left(i \gamma^{\mu} D_{\mu}-m\right) \psi_{n}-\frac{1}{4} F_{\mu \nu}^{a} F^{a \mu \nu}-\frac{1}{2 \xi}\left(\partial^{\mu} A_{\mu}^{a}\right)^{2}+\partial^{\mu} \bar{c}^{a}\left(\partial_{\mu} \delta^{a c}+g f^{a b c} A_{\mu}^{b}\right) c^{c}
$$




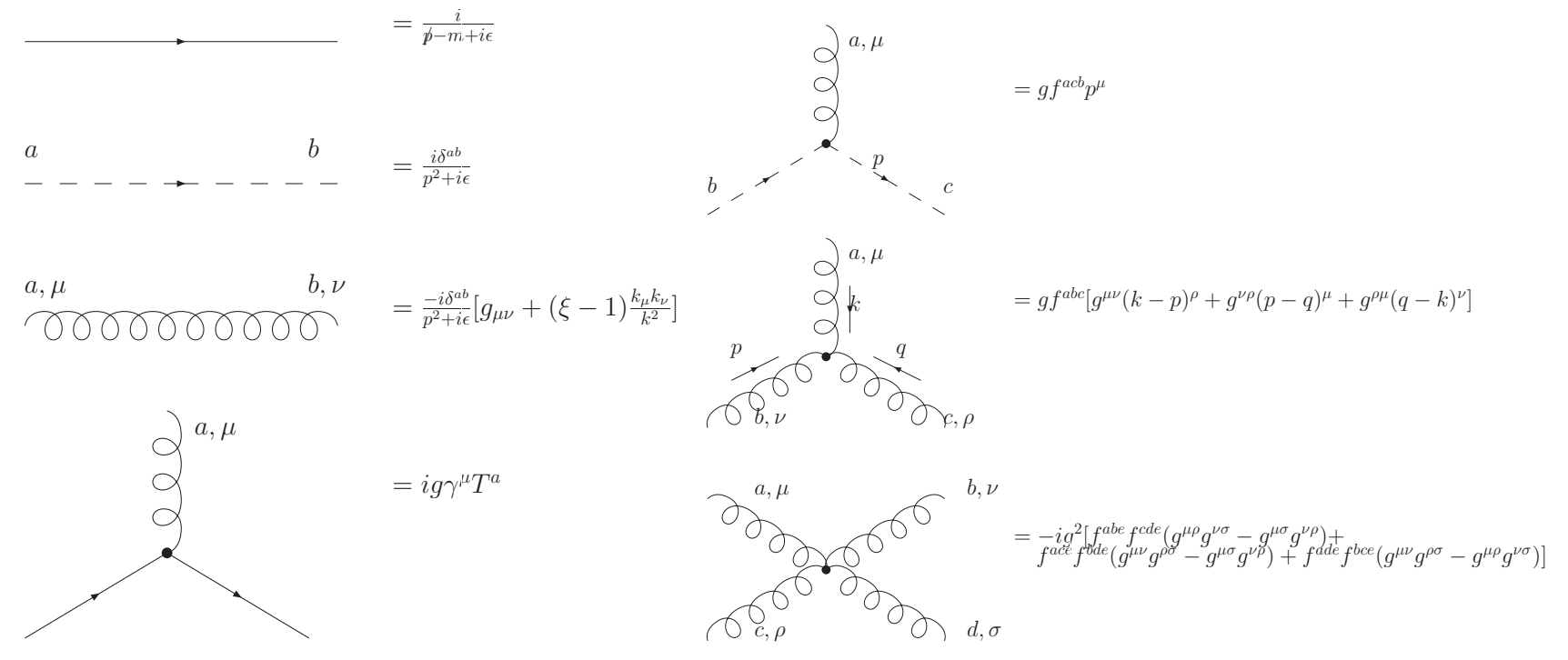

\section{APPENDIX C: CALCULATION OF RENORMALIZATION CONSTANTS IN LOOP REGULARIZATION}

\section{1. fermion self-energy diagram}

There is only one diagram which contribute to the one-loop renormalization for the fermion fields strength as shown in Fig.2.

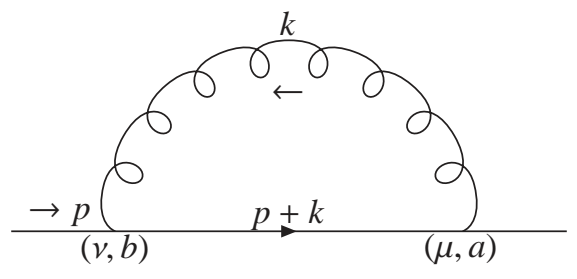

Fig.2.

Following the Feynman rules given in Appendix B, we write down the Feynman integral corresponding to this diagram (for simplification we will ignore the $i \epsilon$ prescription in the propagators throughout this paper, this dose not make any confusion if we keep the prescription in mind):

$$
\begin{aligned}
L(2) & =\int \frac{d^{4} k}{(2 \pi)^{4}}\left(i g \gamma_{\mu} T_{a}\right) \frac{i}{(\not p+\not k)-m} \frac{-i \delta^{a b}}{k^{2}}\left[g^{\mu \nu}+(\xi-1) \frac{k^{\mu} k^{\nu}}{k^{2}}\right]\left(i g \gamma_{\nu} T^{a}\right) \\
& =\int \frac{d^{4} k}{(2 \pi)^{4}}\left(-g^{2} T^{a} T^{b} \delta^{a b}\right) \gamma_{\mu} \frac{\not p+\not k+m}{(p+k)^{2}-m^{2}} \gamma_{\nu} \frac{1}{k^{2}}\left[g^{\mu \nu}+(\xi-1) \frac{k^{\mu} k^{\nu}}{k^{2}}\right] \\
& =\left(-g^{2} C_{2}\right) \int \frac{d^{4} k}{(2 \pi)^{4}} \gamma_{\mu} \frac{\not p+\not k+m}{(p+m)^{2}-m^{2}} \gamma_{\nu}\left(\frac{g^{\mu \nu}}{k^{2}}+\frac{(\xi-1) k^{\mu} k^{\nu}}{k^{4}}\right)
\end{aligned}
$$

we now apply the Feynman parameter method to the denominators in the integral in order to squeeze those denominator factors into a single quadratic polynomial in $k$. Then we can get:

$$
\begin{aligned}
L(2)= & \left(-g^{2} C_{2}\right) \int_{0}^{1} d x_{1} \int \frac{d^{4} k}{(2 \pi)^{4}} \gamma_{\mu}(\not p+\not k+m) \gamma_{\nu}\left(\frac{\Gamma(2) g^{\mu \nu}}{\Gamma(1) \Gamma(1)\left[\left(1-x_{1}\right)\left((p+k)^{2}-m^{2}\right)+x_{1} k^{2}\right]^{2}}+\right. \\
& \left.\frac{\Gamma(3) x_{1}(\xi-1) k^{\mu} k^{\nu}}{\Gamma(1) \Gamma(2)\left[\left(1-x_{1}\right)\left((p+k)^{2}-m^{2}\right)+x_{1} k^{2}\right]^{3}}\right)
\end{aligned}
$$




$$
\begin{aligned}
= & \left(-g^{2} C_{2}\right) \int_{0}^{1} d x_{1} \int \frac{d^{4} k}{(2 \pi)^{4}} \gamma_{\mu}(\not p+\not k+m) \gamma_{\nu}\left(\frac{g^{\mu \nu}}{\left(k^{2}-M_{2}^{2}\right)^{2}}+\right. \\
& \left.\frac{2 x_{1}(\xi-1)\left(k-\left(1-x_{1}\right) p\right)^{\mu}\left(k-\left(1-x_{1}\right) p\right)^{\nu}}{\left(k^{2}-M_{2}^{2}\right)^{3}}\right)
\end{aligned}
$$

where we have shifted the integrating variable by a constant to complete the square in the denominators and have introduced the notation $M_{2}^{2} \equiv\left(1-x_{1}\right) m^{2}-x_{1}\left(1-x_{1}\right) p^{2}$. Then the divergent part can be extracted to be:

$$
\begin{aligned}
L(2)_{d i v}= & \left(-g^{2} C_{2}\right) \int_{0}^{1} d x_{1} \int \frac{d^{4} k}{(2 \pi)^{4}}\left(\frac{g^{\mu \nu} \gamma_{\mu}\left(x_{1} \not p+m\right) \gamma_{\nu}}{\left(k^{2}-M_{2}^{2}\right)^{2}}+\frac{2 x_{1}(\xi-1) k^{\mu} k^{\nu} \gamma_{\mu}\left(x_{1} \not p+m\right) \gamma_{\nu}}{\left(k^{2}-M_{2}^{2}\right)^{3}}+\right. \\
& \left.\frac{2 x_{1}(\xi-1)\left(1-x_{1}\right)\left(-k^{\mu} p^{\nu}-k^{\mu} p^{\mu}\right) \gamma_{\mu} \not k \gamma_{\nu}}{\left(k^{2}-M_{2}^{2}\right)^{3}}\right) \\
= & \left(-g^{2} C_{2}\right) \int_{0}^{1} d x_{1}\left[\left(-2 x_{1} \not p+4 m\right) I_{0}+2 \gamma_{\mu}\left(x_{1} \not p+m\right) \gamma_{\nu} x_{1}(\xi-1) I_{0}^{\mu \nu}+\right. \\
& \left.2 x_{1}\left(x_{1}-1\right)(\xi-1) \gamma_{\mu} \gamma_{\alpha} \gamma_{\nu}\left(p^{\mu} I_{0}^{\alpha \nu}+p^{\nu} I_{0}^{\alpha \mu}\right)\right]
\end{aligned}
$$

It is seen that the Feynman integral can be expressed in terms of 1 -fold ILIs $I_{0}$ and $I_{0}^{\mu \nu}$. To regularize this Feynman integral we only need apply the loop regularization prescription to the relevant ILIs. We mention that the explicit forms of all the regularized ILIs have been worked out in [1, 2], what we need here is to use the relation for the regularized ILIs: $I_{0 \mu \nu}^{R}=\frac{1}{4} g_{\mu \nu} I_{0}^{R}$. As a consequence, the regularized divergent parts of the Feynman diagram can be expressed only in term of the regularized scalar divergent ILIs $I_{0}^{R}$

$$
L(2)_{d i v}^{R}=\left(-g^{2} C_{2}\right) \int_{0}^{1} d x_{1}\left\{\left[x_{1}\left(3 x_{1}-4\right)(\xi-1)-2 x_{1}\right] p p+\left[2 x_{1}(\xi-1)+4\right] m\right\} I_{0}^{R}
$$

\section{2. gluon self-energy diagram}

There are four gluon self-energy diagrams as shown in Fig.1, which have been evaluated in [1, 2], the results read:

$$
\begin{aligned}
L_{R \mu \nu}^{a b}= & g^{2} \delta^{a b}\left(p^{2} g_{\mu \nu}-p_{\mu} p_{\nu}\right) \int_{0}^{1} d x\left\{C_{1}\left[1+4 x(1-x)+\frac{1}{2}(1-\xi)\right] I_{0}^{R}\right. \\
& \left.-N_{f} T_{2} 8 x(1-x) I_{0}^{R}(m)-4 C_{1}(1-\xi)\left[1-\frac{1}{8}(1-\xi)\right] x(1-x) p^{2} I_{-2}^{R}\right\}
\end{aligned}
$$

\section{3. ghost self-energy diagram}

There is only one diagram which contributes to the one-loop renormalization of the ghost fields strength as shown in Fig.3.

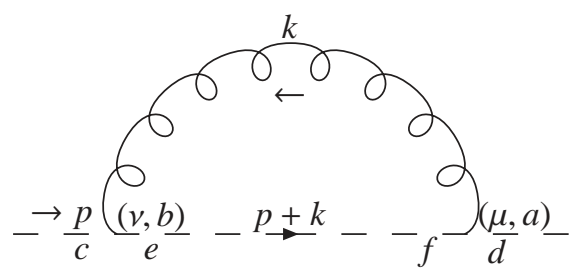

Fig.3.

$$
\begin{aligned}
L(3)^{c d} & =\int \frac{d^{4} k}{(2 \pi)^{4}}\left(g f^{a d f} p_{\mu}\right) \frac{i \delta^{e f}}{(p+k)^{2}} \frac{-i \delta^{a b}}{k^{2}}\left[g^{\mu \nu}+(\xi-1) \frac{k^{\mu} k^{\nu}}{k^{2}}\right]\left(g f^{b e c}(p+k)_{\nu}\right) \\
& =-C_{1} g^{2} \delta^{c d} \int \frac{d^{4} k}{(2 \pi)^{4}} p_{\mu} \frac{1}{(p+k)^{2}}\left[\frac{g^{\mu \nu}}{k^{2}}+(\xi-1) \frac{k^{\mu} k^{\nu}}{k^{4}}\right]\left(p_{\nu}+k_{\nu}\right)
\end{aligned}
$$




$$
\begin{aligned}
& =-C_{1} g^{2} \delta^{c d} \int \frac{d^{4} k}{(2 \pi)^{4}}\left[\frac{p^{2}+\xi p_{\mu} k^{\mu}}{(p+k)^{2} k^{2}}+(\xi-1) \frac{p_{\mu} p_{\nu} k^{\mu} k^{\nu}}{(p+k)^{2} k^{4}}\right] \\
& =-C_{1} g^{2} \delta^{c d} \int_{0}^{1} d x \int \frac{d^{4} k}{(2 \pi)^{4}}\left[\frac{\Gamma(2)}{\Gamma(1) \Gamma(1)} \frac{p^{2}+\xi p_{\mu} k^{\mu}}{\left((1-x)(p+k)^{2}+x k^{2}\right)^{2}}+(\xi-1) \frac{\Gamma(3)}{\Gamma(1) \Gamma(2)} \frac{x p_{\mu} p_{\nu} k^{\mu} k^{\nu}}{\left((1-x)(p+k)^{2}+x k^{2}\right)^{3}}\right] \\
& =-C_{1} g^{2} \delta^{c d} \int_{0}^{1} d x \int \frac{d^{4} k}{(2 \pi)^{4}}\left[\frac{p^{2}+\xi p_{\mu}(k-(1-x) p)^{\mu}}{\left(k^{2}-M_{3}^{2}\right)^{2}}+\frac{2 x(\xi-1) p_{\mu} p_{\nu}(k-(1-x) p)^{\mu}(k-(1-x) p)^{\nu}}{\left(k^{2}-M_{3}^{2}\right)^{3}}\right] \quad(\mathrm{C} 6)
\end{aligned}
$$

where we have introduced the notation $M_{3}^{2}=-x(1-x) p^{2}$. Thus the divergent part can be found to be:

$$
\begin{aligned}
L(3)_{d i v}^{c d} & =-C_{1} g^{2} \delta^{c d} \int_{0}^{1} d x \int \frac{d^{4} k}{(2 \pi)^{4}}\left[\frac{(1-\xi(1-x)) p^{2}}{\left(k^{2}-M_{3}^{2}\right)^{2}}+\frac{2 x(\xi-1) p_{\mu} p_{\nu} k^{\mu} k^{\nu}}{\left(k^{2}-M_{3}^{2}\right)^{3}}\right] \\
& =-C_{1} g^{2} \delta^{c d} \int_{0}^{1} d x \int \frac{d^{4} k}{(2 \pi)^{4}}\left[(1-\xi(1-x)) p^{2} I_{0}+2 x(\xi-1) p_{\mu} p_{\nu} I_{0}^{\mu \nu}\right]
\end{aligned}
$$

Applying for the relation $I_{0 \mu \nu}^{R}=\frac{1}{4} g_{\mu \nu} I_{0}^{R}$, all the divergent parts can be expressed in term of $I_{0}^{R}$ and given by

$$
L(3)_{d i v}^{c d R}=-C_{1} g^{2} \delta^{c d} \int_{0}^{1} d x \int \frac{d^{4} k}{(2 \pi)^{4}}\left(x-\left(1-\frac{3}{2} x\right)(\xi-1)\right) p^{2} I_{0}^{R}
$$

\section{4. fermion-gluon vertex renormalization}

Two diagrams including their permutation can contribute to the one-loop renormalization of fermion-gluon vertex. Let's begin with the calculation for Fig.4a.

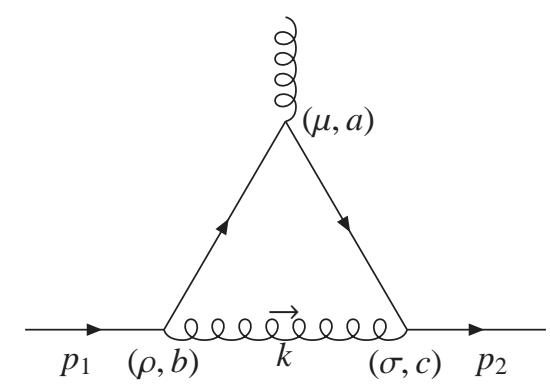

Fig.4a.

$$
\begin{aligned}
L(4 a)_{\mu}^{a}= & \int \frac{d^{4} k}{(2 \pi)^{4}}\left(i g \gamma_{\sigma} T^{c}\right) \frac{i}{\not p_{2}-\not k-m}\left(i g \gamma_{\mu} T^{a}\right) \frac{i}{\not p_{1}-\not k-m}\left(i g \gamma_{\rho} T^{b}\right) \frac{-i \delta^{b c}}{k^{2}}\left[g^{\rho \sigma}+(\xi-1) \frac{k^{\rho} k^{\sigma}}{k^{2}}\right] \\
= & g^{3} T^{b} T^{a} T^{b} \int \frac{d^{4} k}{(2 \pi)^{4}} \gamma_{\sigma} \frac{\not p_{2}-\not k+m}{\left(p_{2}-k\right)^{2}-m^{2}} \gamma_{\mu} \frac{\not p_{1}-\not k+m}{\left(p_{1}-k\right)^{2}-m^{2}} \gamma_{\rho} \frac{1}{k^{2}}\left[g^{\rho \sigma}+(\xi-1) \frac{k^{\rho} k^{\sigma}}{k^{2}}\right] \\
= & \left.g^{3}\left(C_{2}-\frac{1}{2} C_{1}\right) T^{a} \int \frac{d^{4} k}{(2 \pi)^{4}} \frac{\gamma_{\sigma}\left(\not p_{2}-\not k+m\right) \gamma_{\mu}\left(\not p_{1}-\not k+m\right) \gamma_{\rho}}{k^{2}\left[\left(p_{2}-k\right)^{2}-m^{2}\right]\left[\left(p_{1}-k\right)^{2}-m^{2}\right]}+(\xi-1) \frac{k^{\rho} k^{\sigma}}{k^{2}}\right] \\
= & g^{3}\left(C_{2}-\frac{1}{2} C_{1}\right) T^{a} \int_{0}^{1} d x_{1} \int_{0}^{x_{1}} d x_{2} \int \frac{d^{4} k}{(2 \pi)^{4}} \\
& \left\{\frac{\Gamma(3)}{\Gamma(1)^{3}} \frac{\gamma^{\rho}\left(\not p_{2}-\not k+m\right) \gamma_{\mu}\left(\not p_{1}-\not k+m\right) \gamma_{\rho}}{\left\{\left(1-x_{1}\right) k^{2}+\left(x_{1}-x_{2}\right)\left[\left(p_{2}-k\right)^{2}-m^{2}\right]+x_{2}\left[\left(p_{1}-k\right)^{2}-m^{2}\right]\right\}^{3}}\right. \\
& \left.+\frac{\Gamma(4)}{\Gamma(2) \Gamma(1)^{2}} \frac{\left(1-x_{1}\right)(\xi-1) k^{\rho} k^{\sigma} \gamma_{\sigma}\left(\not p_{2}-\not k+m\right) \gamma_{\mu}\left(\not p_{1}-\not k+m\right) \gamma_{\rho}}{\left\{\left(1-x_{1}\right) k^{2}+\left(x_{1}-x_{2}\right)\left[\left(p_{2}-k\right)^{2}-m^{2}\right]+x_{2}\left[\left(p_{1}-k\right)^{2}-m^{2}\right]\right\}^{4}}\right\} \\
= & g^{3}\left(C_{2}-\frac{1}{2} C_{1}\right) T^{a} \int_{0}^{1} d x_{1} \int_{0}^{x_{1}} d x_{2} \int \frac{d^{4} k}{(2 \pi)^{4}}
\end{aligned}
$$




$$
\begin{aligned}
& \left\{\frac{1}{\left(k^{2}-M_{4 a}^{2}\right)^{3}}\left[2 \gamma^{\rho}\left(\not k+\left(x_{1}-x_{2}-1\right) \not p_{2}+x_{2} \not p_{1}-m\right) \gamma_{\mu}\left(\not k+\left(x_{1}-x_{2}\right) \not p_{2}+\left(x_{2}-1\right) \not p_{1}-m\right) \gamma_{\rho}\right]\right. \\
& +\frac{1}{\left(k^{2}-M_{4 a}^{2}\right)^{4}}\left[6\left(1-x_{1}\right)(\xi-1)\left(k+\left(x_{1}-x_{2}\right) p_{2}+x_{2} p_{1}\right)^{\rho}\left(k+\left(x_{1}-x_{2}\right) p_{2}+x_{2} p_{1}\right)^{\sigma} \gamma_{\sigma}\right. \\
& \left.\left.\left(\not k+\left(x_{1}-x_{2}-1\right) \not p_{2}+x_{2} \not p_{1}-m\right) \gamma_{\mu}\left(\not k+\left(x_{1}-x_{2}\right) \not p_{2}+\left(x_{2}-1\right) \not p_{1}-m\right) \gamma_{\rho}\right]\right\}
\end{aligned}
$$

with definition: $M_{4 a}^{2}=-\left(x_{1}-x_{2}\right)\left(1-x_{1}+x_{2}\right) p_{2}^{2}+x_{2}\left(1-x_{2}\right) p_{1}^{2}-2 x_{2}\left(x_{1}-x_{2}\right) p_{1} \cdot p_{2}-x_{1} m^{2}$, and the divergent part is found to be:

$$
\begin{aligned}
L(4 a)_{\mu ; d i v}^{a}= & g^{3}\left(C_{2}-\frac{1}{2} C_{1}\right) T^{a} \int_{0}^{1} d x_{1} \int_{0}^{x_{1}} d x_{2} \int \frac{d^{4} k}{(2 \pi)^{4}}\left[2 \frac{\gamma^{\rho} \not k \gamma_{\mu} \not k \gamma_{\rho}}{\left(k^{2}-M_{4 a}^{2}\right)^{3}}\right. \\
& \left.+6\left(1-x_{1}\right)(\xi-1) \frac{\not k \not k \gamma_{\mu} \not k, k}{\left(k^{2}-M_{4 a}^{2}\right)^{4}}\right] \\
= & g^{3}\left(C_{2}-\frac{1}{2} C_{1}\right) T^{a} \int_{0}^{1} d x_{1} \int_{0}^{x_{1}} d x_{2} \int \frac{d^{4} k}{(2 \pi)^{4}}\left[2 \frac{-4 k_{\mu} k_{\alpha} \gamma^{\alpha}+2 k^{2} \gamma_{\mu}}{\left(k^{2}-M_{4 a}^{2}\right)^{3}}\right. \\
& \left.+6\left(1-x_{1}\right)(\xi-1) \frac{k^{4} \gamma_{\mu}}{\left(k^{2}-M_{4 a}^{2}\right)^{4}}\right] \\
\sim & g^{3}\left(C_{2}-\frac{1}{2} C_{1}\right) T^{a} \int_{0}^{1} d x_{1} \int_{0}^{x_{1}} d x_{2}\left[-8 \gamma^{\alpha} I_{0 \mu \alpha}+4 \gamma_{\mu} I_{0}+6\left(1-x_{1}\right)(\xi-1) \gamma_{\mu} I_{0}\right]
\end{aligned}
$$

By adopting the relation $I_{0 \mu \nu}^{R}=\frac{1}{4} g_{\mu \nu} I_{0}^{R}$ the divergent parts can be expressed in term of $I_{0}^{R}$

$$
L(4 a)_{\mu ; d i v}^{a R}=g^{3}\left(C_{2}-\frac{1}{2} C_{1}\right) T^{a} \int_{0}^{1} d x_{1} \int_{0}^{x_{1}} d x_{2}\left[2+6\left(1-x_{1}\right)(\xi-1)\right] \gamma_{\mu} I_{0}^{R}
$$

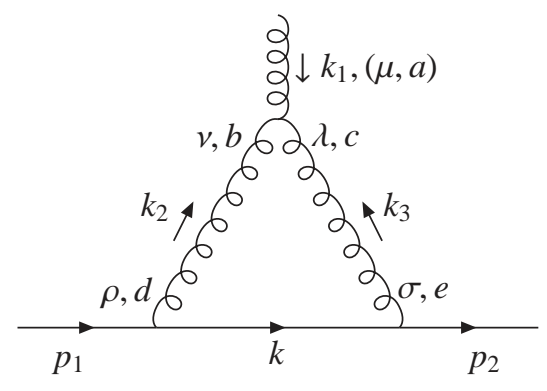

Fig.4b.

The second diagram (Fig.4b) that contributes to the fermion-gluon vertex has the following form

$$
\begin{aligned}
L(4 b)_{\mu}^{a}= & \int \frac{d^{4} k}{(2 \pi)^{4}}\left(i g \gamma_{\sigma} T^{e}\right) \frac{i}{\not k-m}\left(i g \gamma_{\rho} T^{d}\right) \frac{-i \delta^{e c}}{k_{3}^{2}}\left[g^{\sigma \lambda}+(\xi-1) \frac{k_{3}^{\sigma} k_{3}^{\lambda}}{k_{3}^{2}}\right] \times \\
& g f^{a b c}\left[\left(k_{1}-k_{2}\right)_{\lambda} g_{\mu \nu}+\left(k_{2}-k_{3}\right)_{\mu} g_{\nu \lambda}+\left(k_{3}-k_{1}\right)_{\nu} g_{\mu \lambda}\right] \frac{-i \delta^{b d}}{k_{2}^{2}}\left[g^{\nu \rho}+(\xi-1) \frac{k_{2}^{\nu} k_{2}^{\rho}}{k_{2}^{2}}\right] \\
= & \frac{1}{2} g^{3} C_{1} T^{a} \int \frac{d^{4} k}{(2 \pi)^{4}} \gamma_{\sigma} \frac{\not k+m}{k^{2}-m^{2}} \gamma_{\rho}\left[\frac{g^{\sigma \lambda}}{k_{3}^{2}}+(\xi-1) \frac{k_{3}^{\sigma} k_{3}^{\lambda}}{k_{3}^{4}}\right] \times \\
& {\left[\left(k_{1}-k_{2}\right)_{\lambda} g_{\mu \nu}+\left(k_{2}-k_{3}\right)_{\mu} g_{\nu \lambda}+\left(k_{3}-k_{1}\right)_{\nu} g_{\mu \lambda}\right]\left[\frac{g^{\nu \rho}}{k_{2}^{2}}+(\xi-1) \frac{k_{2}^{\nu} k_{2}^{\rho}}{k_{2}^{4}}\right] } \\
= & \frac{1}{2} g^{3} C_{1} T^{a} \int \frac{d^{4} k}{(2 \pi)^{4}} \gamma_{\sigma} \frac{\not k+m}{k^{2}-m^{2}} \gamma_{\rho}\left[\left(k_{1}-k_{2}\right)_{\lambda} g_{\mu \nu}+\left(k_{2}-k_{3}\right)_{\mu} g_{\nu \lambda}+\left(k_{3}-k_{1}\right)_{\nu} g_{\mu \lambda}\right] \times \\
& {\left[\frac{g^{\sigma \lambda} g^{\nu \rho}}{k_{3}^{2} k_{2}^{2}}+(\xi-1) \frac{g^{\sigma \lambda} k_{2}^{\nu} k_{2}^{\rho}}{k_{3}^{2} k_{2}^{4}}+(\xi-1) \frac{g^{\nu \rho} k_{3}^{\sigma} k_{3}^{\lambda}}{k_{3}^{4} k_{2}^{2}}+(\xi-1)^{2} \frac{k_{3}^{\sigma} k_{3}^{\lambda} k_{2}^{\nu} k_{2}^{\rho}}{k_{3}^{4} k_{2}^{4}}\right] } \\
= & \frac{1}{2} g^{3} C_{1} T^{a} \int_{0}^{1} d x_{1} \int_{0}^{x_{1}} d x_{2} \int \frac{d^{4} k}{(2 \pi)^{4}} \gamma_{\sigma}(\not k+m) \gamma_{\rho}\left[\left(k_{1}-k_{2}\right)_{\lambda} g_{\mu \nu}+\left(k_{2}-k_{3}\right)_{\mu} g_{\nu \lambda}+\left(k_{3}-k_{1}\right)_{\nu} g_{\mu \lambda}\right] \times
\end{aligned}
$$




$$
\begin{aligned}
& {\left[\frac{\Gamma(3)}{\Gamma(1)^{3}} \frac{g^{\sigma \lambda} g^{\nu \rho}}{\left[\left(1-x_{1}\right)\left(k^{2}-m^{2}\right)+\left(x_{1}-x_{2}\right) k_{3}^{2}+x_{2} k_{2}^{2}\right]^{3}}+\right.} \\
& +(\xi-1) \frac{\Gamma(4)}{\Gamma(1)^{2} \Gamma(2)} \frac{x_{2} g^{\sigma \lambda} k_{2}^{\nu} k_{2}^{\rho}}{\left[\left(1-x_{1}\right)\left(k^{2}-m^{2}\right)+\left(x_{1}-x_{2}\right) k_{3}^{2}+x_{2} k_{2}^{2}\right]^{4}} \\
& +(\xi-1) \frac{\Gamma(4)}{\Gamma(1)^{2} \Gamma(2)} \frac{\left(x_{1}-x_{2}\right) g^{\nu \rho} k_{3}^{\sigma} k_{3}^{\lambda}}{\left[\left(1-x_{1}\right)\left(k^{2}-m^{2}\right)+\left(x_{1}-x_{2}\right) k_{3}^{2}+x_{2} k_{2}^{2}\right]^{4}}+ \\
& \left.+(\xi-1)^{2} \frac{\Gamma(5)}{\Gamma(1) \Gamma(2)^{2}} \frac{\left(x_{1}-x_{2}\right) x_{2} k_{3}^{\sigma} k_{3}^{\lambda} k_{2}^{\nu} k_{2}^{\rho}}{\left[\left(1-x_{1}\right)\left(k^{2}-m^{2}\right)+\left(x_{1}-x_{2}\right) k_{3}^{2}+x_{2} k_{2}^{2}\right]^{5}}\right]
\end{aligned}
$$

Introducing the notation $M_{4 b}^{2}=-\left(x_{1}-x_{2}\right)\left(1-x_{1}+x_{2}\right) p_{2}^{2}+x_{2}\left(1-x_{2}\right) p_{1}^{2}-2 x_{2}\left(x_{1}-x_{2}\right) p_{1} \cdot p_{2}-x_{1} m^{2}$, the divergent part can be written down as follows:

$$
\begin{aligned}
L(4 b)_{\mu ; d i v}^{a}= & \frac{1}{2} g^{3} C_{1} T^{a} \int_{0}^{1} d x_{1} \int_{0}^{x_{1}} d x_{2} \int \frac{d^{4} k}{(2 \pi)^{4}} \gamma_{\sigma} \not k \gamma_{\rho}\left(k_{\lambda} g_{\mu \nu}-2 k_{\mu} g_{\nu \lambda}+k_{\nu} g_{\mu \lambda}\right) \times \\
& {\left[\frac{2 g^{\sigma \lambda} g^{\nu \rho}}{\left(k^{2}-M_{4 b}^{2}\right)^{3}}+(\xi-1) \frac{6 x_{2} g^{\sigma \lambda} k^{\nu} k^{\rho}}{\left(k^{2}-M_{4 b}^{2}\right)^{4}}+(\xi-1) \frac{6\left(x_{1}-x_{2}\right) g^{\nu \rho} k^{\sigma} k^{\lambda}}{\left(k^{2}-M_{4 b}^{2}\right)^{4}}+(\xi-1)^{2} \frac{24\left(x_{1}-x_{2}\right) x_{2} k^{\sigma} k^{\lambda} k^{\nu} k^{\rho}}{\left(k^{2}-M_{4 b}^{2}\right)^{5}}\right] } \\
= & \frac{1}{2} g^{3} C_{1} T^{a} \int_{0}^{1} d x_{1} \int_{0}^{x_{1}} d x_{2}\left[2 \gamma^{\lambda} \gamma^{\alpha} \gamma_{\mu} I_{0 \alpha \lambda}+2 \gamma_{\mu} \gamma^{\alpha} \gamma^{\nu} I_{0 \alpha \nu}-4 \gamma^{\lambda} \gamma^{\alpha} \gamma_{\lambda} I_{0 \alpha \mu}+6 x_{2}(\xi-1) \gamma^{\lambda} \gamma^{\alpha} \gamma^{\rho} I_{0 \alpha \rho \lambda \mu}\right. \\
& -12 x_{2}(\xi-1) \gamma^{\lambda} \gamma^{\alpha} \gamma^{\rho} I_{0 \alpha \rho \lambda \mu}+6 x_{2}(\xi-1) \gamma_{\mu} \gamma^{\alpha} \gamma^{\rho} I_{0 \alpha \rho}+6\left(x_{1}-x_{2}\right)(\xi-1) \gamma^{\sigma} \gamma^{\alpha} \gamma_{\mu} I_{0 \alpha \sigma} \\
& \left.-12\left(x_{1}-x_{2}\right)(\xi-1) \gamma^{\sigma} \gamma^{\alpha} \gamma^{\nu} I_{0 \alpha \sigma \mu \nu}+6\left(x_{1}-x_{2}\right)(\xi-1) \gamma^{\sigma} \gamma^{\alpha} \gamma^{\nu} I_{0 \alpha \sigma \mu \nu}+0\right] \\
= & g^{3} C_{1} T^{a} \int_{0}^{1} d x_{1} \int_{0}^{x_{1}} d x_{2}\left[4 \gamma^{\alpha} I_{0 \alpha \mu}+\left(3\left(x_{1}-x_{2}\right)(\xi-1)+1\right) \gamma^{\lambda} \gamma^{\sigma} \gamma_{\mu} I_{0 \lambda \alpha}+\right. \\
& \left.+\left(3 x_{2}(\xi-1)+1\right) \gamma_{\mu} \gamma^{\lambda} \gamma^{\alpha} I_{0 \lambda \alpha}-3 x_{1}(\xi-1) \gamma^{\alpha} \gamma^{\sigma} \gamma^{\rho} I_{0 \alpha \sigma \rho \mu}\right]
\end{aligned}
$$

Using the relations $I_{0 \mu \nu}^{R}=\frac{1}{4} g_{\mu \nu} I_{0}^{R}$ and $I_{0 \rho \sigma \alpha \beta}^{R}=\frac{1}{24}\left(g_{\rho \sigma} g_{\alpha \beta}+g_{\rho \alpha} g_{\sigma \beta}+g_{\rho \beta} g_{\sigma \alpha}\right) I_{0}^{R}$, the divergent parts can be simplified to be

$$
L(4 b)_{\mu ; d i v}^{a R}=g^{3} C_{1} T^{a} \gamma_{\mu} \int_{0}^{1} d x_{1} \int_{0}^{x_{1}} d x_{2}\left[3+\frac{9}{4} x_{1}(\xi-1)\right] I_{0}^{R}
$$

\section{5. ghost-gluon vertex renormalization}

There are two diagrams which contribute to the one-loop renormalization of the ghost-gluon vertex. Let's consider the first diagram (Fig.5a)

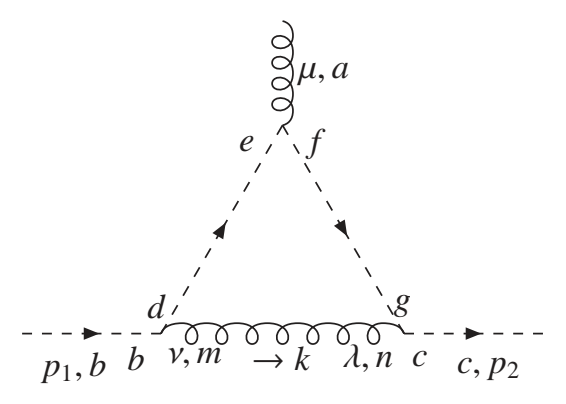

Fig.5a.

$$
\begin{aligned}
L(5 a)_{\mu}^{a c b}= & \int \frac{d^{4} k}{(2 \pi)^{4}}\left(g f^{n c g} p_{2 \lambda}\right) \frac{i \delta^{f g}}{\left(p_{2}-k\right)^{2}}\left(g f^{a f e}\left(p_{2}-k\right)_{\mu}\right) \frac{i \delta^{e d}}{\left(p_{1}-k\right)^{2}}\left(g f^{m d b}\left(p_{1}-k\right)_{\nu}\right) \\
& \frac{-i \delta^{m n}}{k^{2}}\left[g^{\nu \lambda}+(\xi-1) \frac{k^{\nu} k^{\lambda}}{k^{2}}\right]
\end{aligned}
$$




$$
\begin{aligned}
= & -\frac{i}{2} g^{3} C_{1} f^{a c b} \int \frac{d^{4} k}{(2 \pi)^{4}} p_{2 \lambda} \frac{\left(p_{2}-k\right)_{\mu}}{\left(p_{2}-k\right)^{2}} \frac{\left(p_{1}-k\right)_{\nu}}{\left(p_{1}-k\right)^{2}}\left[\frac{g^{\nu \lambda}}{k^{2}}+(\xi-1) \frac{k^{\nu} k^{\lambda}}{k^{4}}\right] \\
= & -\frac{i}{2} g^{3} C_{1} f^{a c b} \int \frac{d^{4} k}{(2 \pi)^{4}}\left[\frac{\left(p_{1} \cdot p_{2}\right) p_{2 \mu}-\left(p_{1} \cdot p_{2}\right) k_{\mu}-\xi p_{2}^{\nu} p_{2 \mu} k_{\nu}+\xi p_{2}^{\nu} k_{\mu} k_{\nu}}{\left(p_{2}-k\right)^{2}\left(p_{1}-k\right)^{2} k^{2}}+\right. \\
& \left.+(\xi-1) \frac{p_{2 \lambda} p_{2 \mu} p_{1 \nu} k^{\nu} k^{\lambda}-p_{2 \lambda} p_{1 \nu} k_{\mu} k^{\nu} k^{\lambda}}{\left(p_{2}-k\right)^{2}\left(p_{1}-k\right)^{2} k^{4}}\right] \\
= & -\frac{i}{2} g^{3} C_{1} f^{a c b} \int_{0}^{1} d x_{1} \int_{0}^{x_{1}} d x_{2} \int \frac{d^{4} k}{(2 \pi)^{4}}\left[\frac{\Gamma(3)}{\Gamma(1)^{3}} \frac{\left(p_{1} \cdot p_{2}\right) p_{2 \mu}-\left(p_{1} \cdot p_{2}\right) k_{\mu}-\xi p_{2}^{\nu} p_{2 \mu} k_{\nu}+\xi p_{2}^{\nu} k_{\mu} k_{\nu}}{\left.\left(1-x_{1}\right)\left(p_{2}-k\right)^{2}+\left(x_{1}-x_{2}\right)\left(p_{1}-k\right)^{2}+x_{2} k^{2}\right]^{3}}+\right. \\
& \left.+(\xi-1) \frac{\Gamma(4)}{\Gamma(1) \Gamma(1) \Gamma(2)} \frac{x_{2}\left(p_{2 \lambda} p_{2 \mu} p_{1 \nu} k^{\nu} k^{\lambda}-p_{2 \lambda} p_{1 \nu} k_{\mu} k^{\nu} k^{\lambda}\right)}{\left[\left(1-x_{1}\right)\left(p_{2}-k\right)^{2}+\left(x_{1}-x_{2}\right)\left(p_{1}-k\right)^{2}+x_{2} k^{2}\right]^{4}}\right]
\end{aligned}
$$

Taking the notation $M_{5 a}^{2}=-\left(1-x_{1}\right) x_{1} p_{2}^{2}+\left(x_{1}-x_{2}\right)\left(1-x_{1}+x_{2}\right) p_{1}^{2}-2\left(1-x_{1}\right)\left(x_{1}-x_{2}\right) p_{1} \cdot p_{2}$, the divergent part is given by

$$
\begin{aligned}
L(5 a)_{\mu ; d i v}^{a c b} & =-\frac{i}{2} g^{3} C_{1} f^{a c b} \int_{0}^{1} d x_{1} \int_{0}^{x_{1}} d x_{2} \int \frac{d^{4} k}{(2 \pi)^{4}} \frac{2 \xi p_{2}^{\nu} k_{\mu} k_{\nu}}{\left(k^{2}-M_{5 a}^{2}\right)^{3}} \\
& =-\frac{i}{2} g^{3} C_{1} f^{a c b} \int_{0}^{1} d x_{1} \int_{0}^{x_{1}} d x_{2} 2 \xi p_{2}^{\nu} I_{0 \mu \nu}
\end{aligned}
$$

with the relation $I_{0 \mu \nu}^{R}=\frac{1}{4} g_{\mu \nu} I_{0}^{R}$, the divergent part is simply given by

$$
L(5 a)_{\mu ; d i v}^{a c b R}=-\frac{i}{2} g^{3} C_{1} f^{a c b} \int_{0}^{1} d x_{1} \int_{0}^{x_{1}} d x_{2} \frac{1}{2} \xi p_{2 \mu} I_{0}^{R}
$$

We now evaluate the second diagram (fig.5b) which contributes to the ghost-gluon vertex as:

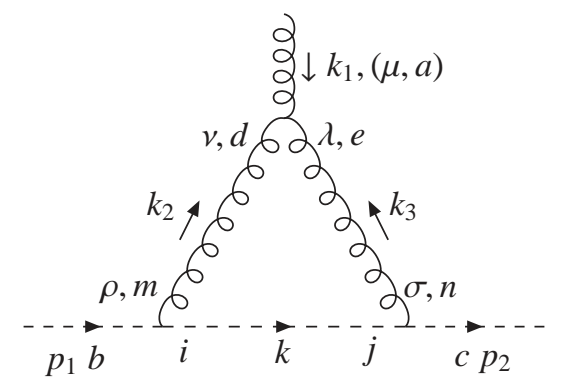

Fig.5b.

$$
\begin{aligned}
L(5 b)_{\mu}^{a c b}= & \int \frac{d^{4} k}{(2 \pi)^{4}}\left(g f^{n c j} p_{2 \sigma}\right) \frac{-i \delta^{e n}}{k_{3}^{2}}\left[g^{\lambda \sigma}+(\xi-1) \frac{k_{3}^{\lambda} k_{3}^{\sigma}}{k_{3}^{2}}\right]\left(g f^{a d e}\right)\left[\left(k_{1}-k_{2}\right)_{\lambda} g_{\mu \nu}+\left(k_{2}-k_{3}\right)_{\mu} g_{\nu \lambda}+\left(k_{3}-k_{1}\right)_{\nu} g_{\lambda \mu}\right] \\
& \frac{-i \delta^{d m}}{k_{2}^{2}}\left[g^{\rho \nu}+(\xi-1) \frac{k_{2}^{\rho} k_{2}^{\nu}}{k_{2}^{2}}\right] \frac{i \delta^{i j}}{k^{2}}\left(g f^{m i b} k_{\rho}\right) \\
= & -\frac{i}{2} g^{3} C_{1} f^{a c b} \int d k \frac{p_{2 \sigma} k_{\rho}}{k^{2}}\left[\frac{g^{\lambda \sigma}}{k_{3}^{2}}+(\xi-1) \frac{k_{3}^{\lambda} k_{3}^{\sigma}}{k_{3}^{4}}\right]\left[\frac{g^{\rho \nu}}{k_{2}^{2}}+(\xi-1) \frac{k_{2}^{\rho} k_{2}^{\nu}}{k_{2}^{4}}\right] \times \\
& {\left[\left(k_{1}-k_{2}\right)_{\lambda} g_{\mu \nu}+\left(k_{2}-k_{3}\right)_{\mu} g_{\nu \lambda}+\left(k_{3}-k_{1}\right)_{\nu} g_{\lambda \mu}\right] } \\
= & -\frac{i}{2} g^{3} C_{1} f^{a c b} \int d k\left[\left(k_{1}-k_{2}\right)_{\lambda} g_{\mu \nu}+\left(k_{2}-k_{3}\right)_{\mu} g_{\nu \lambda}+\left(k_{3}-k_{1}\right)_{\nu} g_{\lambda \mu}\right] \times \int_{0}^{1} d x_{1} \int_{0}^{x_{1}} d x_{2} \\
& {\left[\frac{\Gamma(3)}{\Gamma(1)^{3}} \overline{\left[\left(1-x_{1}\right) k^{2}+\left(x_{1}-x_{2}\right) k_{2}^{2}+x_{2} k_{3}^{2}\right]^{3}}+\frac{\Gamma(4)}{\Gamma(1)^{2} \Gamma(2)} \frac{x_{2}(\xi-1) p_{2 \sigma} k_{\rho} k_{3}^{\lambda} k_{3}^{\sigma} g^{\rho \nu}}{\left[\left(1-x_{1}\right) k^{2}+\left(x_{1}-x_{2}\right) k_{2}^{2}+x_{2} k_{3}^{2}\right]^{4}}\right.} \\
& \left.+\frac{\Gamma(4)}{\Gamma(1)^{2} \Gamma(2)} \frac{\left(x_{1}-x_{2}\right)(\xi-1) p_{2 \sigma} k_{\rho} k_{2}^{\rho} k_{2}^{\nu} g^{\lambda \sigma}}{\left[\left(1-x_{1}\right) k^{2}+\left(x_{1}-x_{2}\right) k_{2}^{2}+x_{2} k_{3}^{2}\right]^{4}}+\frac{\Gamma(5)}{\Gamma(1) \Gamma(2)^{2}} \frac{x_{2}\left(x_{1}-x_{2}\right)(\xi-1)^{2} p_{2 \sigma} k_{\rho} k_{3}^{\lambda} k_{3}^{\sigma} k_{2}^{\rho} k_{2}^{\nu}}{\left[\left(1-x_{1}\right) k^{2}+\left(x_{1}-x_{2}\right) k_{2}^{2}+x_{2} k_{3}^{2}\right]^{5}}\right]
\end{aligned}
$$


Introducing the notation $M_{5 b}^{2}=-\left(x_{1}-x_{2}\right)\left(1-x_{1}+x_{2}\right) p_{1}^{2}+x_{2}\left(1-x_{2}\right) p_{2}^{2}-2 x_{2}\left(x_{1}-x_{2}\right) p_{1} \cdot p_{2}$, the divergent part can be expressed as:

$$
\begin{aligned}
L(5 b)_{\mu ; d i v}^{a c b}= & -\frac{i}{2} g^{3} C_{1} f^{a c b} \int_{0}^{1} d x_{1} \int_{0}^{x_{1}} d x_{2} \int \frac{d^{4} k}{(2 \pi)^{4}}\left[k_{\lambda} g_{\mu \nu}-2 k_{\mu} g_{\nu \lambda}+k_{\nu} g_{\lambda \mu}\right]\left[\frac{2 p_{2 \sigma} k_{\rho} g^{\lambda \sigma} g^{\rho \nu}}{\left(k^{2}-M_{5 b}^{2}\right)^{3}}+\right. \\
& \left.\frac{6 x_{2}(\xi-1) p_{2 \sigma} k_{\rho} k^{\lambda} k^{\sigma} g^{\rho \nu}}{\left(k^{2}-M_{5 b}^{2}\right)^{4}}+\frac{6\left(x_{1}-x_{2}\right)(\xi-1) p_{2 \sigma} k_{\rho} k^{\rho} k^{\nu} g^{\lambda \sigma}}{\left(k^{2}-M_{5 b}^{2}\right)^{4}}+\frac{24 x_{2}\left(x_{1}-x_{2}\right)(\xi-1)^{2} p_{2 \sigma} k_{\rho} k^{\rho} k^{\nu} k^{\lambda} k^{\sigma}}{\left(k^{2}-M_{5 b}^{2}\right)^{5}}\right] \\
= & -\frac{i}{2} g^{3} C_{1} f^{a c b} \int_{0}^{1} d x_{1} \int_{0}^{x_{1}} d x_{2} \int \frac{d^{4} k}{(2 \pi)^{4}}\left[\frac{-2 p_{2}^{\sigma} k_{\sigma} k_{\mu}}{\left(k^{2}-M_{5 b}^{2}\right)^{3}}+\frac{2 p_{2 \mu}}{\left(k^{2}-M_{5 b}^{2}\right)^{3}}+0+\frac{-6\left(x_{1}-x_{2}\right)(\xi-1) p_{2}^{\sigma} k_{\sigma} k_{\mu}}{\left(k^{2}-M_{5 b}^{2}\right)^{3}}\right. \\
& \left.+\frac{6\left(x_{1}-x_{2}\right)(\xi-1) p_{2 \mu}}{\left(k^{2}-M_{5 b}^{2}\right)^{3}}+0\right] \\
= & -i g^{3} C_{1} f^{a c b} \int_{0}^{1} d x_{1} \int_{0}^{x_{1}} d x_{2}\left[\left(3\left(x_{2}-x_{1}\right)(\xi-1)-1\right) p_{2}^{\sigma} I_{0 \sigma \mu}+\left(-3\left(x_{2}-x_{1}\right)(\xi-1)+1\right) p_{2 \mu} I_{0}\right]
\end{aligned}
$$

with the relation $I_{0 \mu \nu}^{R}=\frac{1}{4} g_{\mu \nu} I_{0}^{R}$, all the divergent parts can be expressed in term of $I_{0}^{R}$

$$
L(5 b)_{\mu ; d i v}^{a c b R}=-\frac{3 i}{4} g^{3} C_{1} f^{a c b} \int_{0}^{1} d x_{1} \int_{0}^{x_{1}} d x_{2}\left(3\left(x_{1}-x_{2}\right)(\xi-1)+1\right) p_{2 \mu} I_{0}^{R}
$$

\section{6. three-gluon vertex renormalization}

Four diagrams including their permutation graphs can contribute to the one-loop renormalization of three-gluon vertex, let's evaluate each of them. We begin with the first graph (Fig.6a)

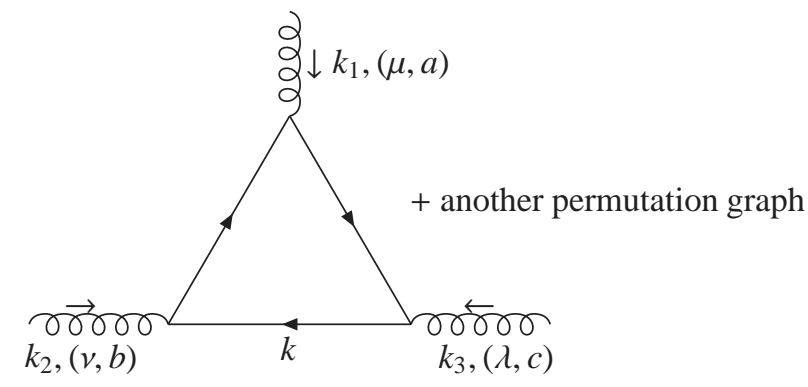

Fig.6a.

which is calculated via the following form

$$
\begin{aligned}
& L(6 a)_{\mu \nu \lambda}^{a b c}=-\operatorname{Tr} \int \frac{d^{4} k}{(2 \pi)^{4}} i g \gamma_{\mu} T^{a} \frac{1}{\not k+\not k k_{2}-m} i g \gamma_{\nu} T_{b} \frac{i}{\not k-m} i g \gamma_{\lambda} T^{c} \frac{i}{\not k-\not k k_{3}-m} \\
& --\operatorname{Tr} \int \frac{d^{4} k}{(2 \pi)^{4}} i g \gamma_{\mu} T^{a} \frac{1}{-\not k+\not k k_{3}-m} i g \gamma_{\nu} T_{b} \frac{i}{1 \not k-m} i g \gamma_{\lambda} T^{c} \frac{i}{-\not k-\not k_{2}-m} \\
& =-\left[\operatorname{Tr}\left(T^{a} T^{b} T^{c}\right)+\operatorname{Tr}\left(T^{a} T^{c} T^{b}\right)\right] \int \frac{d^{4} k}{(2 \pi)^{4}} \operatorname{tr}\left(i g \gamma_{\mu} \frac{1}{\not k+\not k_{2}-m} i g \gamma_{\nu} \frac{i}{\not k-m} i g \gamma_{\lambda} \frac{i}{\not k-\not k} k_{3}-m\right) \\
& =-\operatorname{Tr}\left(i f_{a b d} T^{d} T^{c}\right) \int \frac{d^{4} k}{(2 \pi)^{4}}\left(-g^{3}\right) \frac{\operatorname{tr}\left[\gamma_{m u}\left(\not k+\not k_{2}+m\right) \gamma_{\nu}(\not k+m) \gamma_{\lambda}\left(\not k-\not k k_{3}-m\right)\right]}{\left[(k+k+)^{2}-m^{2}\right]\left[k^{2}-m^{2}\right]\left[\left(k-k_{3}\right)^{2}-m^{2}\right]} \\
& =i g^{3} f^{g b c} T_{2} \int \frac{d^{4} k}{(2 \pi)^{4}} \int_{0}^{1} d x_{1} \int_{0}^{x_{1}} d x_{2} \frac{\Gamma(3)}{\Gamma(1)^{3}} \\
& \frac{\operatorname{tr}\left[\gamma_{m u}\left(\not k+\not k k_{2}+m\right) \gamma_{\nu}(\not k+m) \gamma_{\lambda}\left(\not k-\not k k_{3}-m\right)\right]}{\left\{\left(k^{2}-m^{2}\right)\left(1-x_{1}\right)+\left[\left(k+k+{ }_{2}\right)^{2}-m^{2}\right]\left(x_{1}-x_{2}\right)+\left[\left(k-k_{3}\right)^{2}-m^{2}\right] x_{2}\right\}^{3}} \\
& =2 i g^{3} f^{a b c} T_{2} \int \frac{d^{4} k}{(2 \pi)^{4}} \int_{0}^{1} d x_{1} \int_{0}^{x_{1}} d x_{2} \frac{\Gamma(3)}{\Gamma(1)^{3}}
\end{aligned}
$$




$$
\begin{aligned}
& \frac{\operatorname{tr}\left[\gamma_{m u}\left(\not k+\not k k_{2}+m\right) \gamma_{\nu}(\not k+m) \gamma_{\lambda}\left(\not k-\not k k_{3}-m\right)\right]}{\left[\left(k+\left(x_{1}-x_{2}\right) k_{2}-x_{2} k_{3}\right)^{2}-m^{2}+\left(x_{1}-x_{2}\right)\left(1-x_{1}+x_{2}\right) k_{2}^{2}+x_{2}\left(1-x_{2}\right) k_{3}^{2}\right]^{3}} \\
= & 2 i g^{3} f^{a b c} T_{2} \int \frac{d^{4} k}{(2 \pi)^{4}} \int_{0}^{1} d x_{1} \int_{0}^{x_{1}} d x_{2} \frac{1}{\left(k^{2}-M_{6 a}^{2}\right)^{3}} \times \\
& \operatorname{tr}\left[\gamma_{\mu}\left(\not k-\left(x_{1}-x_{2}\right) \not k_{2}+x_{2} \not k_{3}+\not k_{2}+m\right) \gamma_{\nu}\left(\not k-\left(x_{1}-x_{2}\right) \not k_{2}+x_{2} \not k_{3}+m\right)\right. \\
& \left.\gamma_{\lambda}\left(\not k-\left(x_{1}-x_{2}\right) \not k_{2}+x_{2} \not k_{3}-\not k_{3}-m\right)\right]
\end{aligned}
$$

with $M_{6 a}^{2}=m^{2}-\left(x_{1}-x_{2}\right)\left(1-x_{1}+x_{2}\right) k_{2}^{2}-x_{2}\left(1-x_{2}\right) k_{3}^{2}$. The divergent part is given by

$$
\begin{aligned}
L(6 a)_{\mu \nu \lambda ; d i v}^{a b c}= & 2 i g^{3} f^{a b c} T_{2} \int \frac{d^{4} k}{(2 \pi)^{4}} \int_{0}^{1} d x_{1} \int_{0}^{x_{1}} d x_{2} \frac{1}{\left(k^{2}-M_{6 a}^{2}\right)^{3}} \times \\
& t r\left[\gamma_{\mu} \not k \gamma_{\nu} \not k \gamma_{\lambda}\left(-\left(x_{1}-x_{2}\right) \not k_{2}+x_{2} \not k_{3}-\not k_{3}+m\right)+\right. \\
& +\gamma_{\mu} \not k \gamma_{\nu}\left(-\left(x_{1}-x_{2}\right) \not k_{2}+x_{2} \not k_{3}+m\right) \gamma_{\lambda} \not k+ \\
& \left.+\gamma_{\mu}\left(-\left(x_{1}-x_{2}\right) \not k_{2}+x_{2} \not k_{3}+\not k_{2}+m\right) \gamma_{\nu} \not k \gamma_{\lambda} \not k\right] \\
= & 2 i g^{3} f^{a b c} T_{2} \int \frac{d^{4} k}{(2 \pi)^{4}} \int_{0}^{1} d x_{1} \int_{0}^{x_{1}} d x_{2} \frac{1}{\left(k^{2}-M_{6 a}^{2}\right)^{3}}\left[\left(x_{2}-x_{1}\right) \operatorname{tr}\left(\gamma_{\mu} \not k \gamma_{\nu} \not k \gamma_{\gamma} \not k_{2}\right)+\right. \\
& +\left(x_{2}-1\right) \operatorname{tr}\left(\gamma_{\mu} \not k \gamma_{\nu} \not \gamma_{\gamma} \not k_{3}\right)+\left(x_{2}-x_{1}\right) \operatorname{tr}\left(\gamma_{\mu} \not k \gamma_{\nu} \not k_{2} \gamma_{\gamma} \not k\right)+x_{2} t r\left(\gamma_{\mu} \not k \gamma_{\nu} \not k_{3} \gamma_{\gamma} \not k\right)+ \\
& \left.+\left(x_{2}-x_{1}+1\right) t r\left(\gamma_{\mu} \not k_{2} \gamma_{\nu} \not k \gamma_{\gamma} \not k\right)+x_{2} t r\left(\gamma_{\mu} \not k_{3} \gamma_{\nu} \not k \gamma_{\gamma} \not k\right)\right] \\
= & 2 i g^{3} f^{a b c} T_{2} \int \frac{d^{4} k}{(2 \pi)^{4}} \int_{0}^{1} d x_{1} \int_{0}^{x_{1}} d x_{2} \frac{1}{\left(k^{2}-M_{6 a}^{2}\right)^{3}}\left[4 ( - x _ { 1 } + x _ { 2 } + 1 ) \left(2 k_{2 \mu} k_{\nu} k_{\lambda}+\right.\right. \\
& \left.+2 k_{\mu} k_{2 \nu} k_{\lambda}+k_{2 \lambda} g_{\mu \nu} k^{2}-k_{2 \mu} g_{\nu \lambda} k^{2}-k_{2 \nu} g_{\mu \lambda} k^{2}-2 k_{\lambda} g_{\mu \nu} k \cdot k_{2}\right)+x_{2}\left(2 k_{3 \mu} k_{\nu} k_{\lambda}+\right. \\
& \left.+2 k_{\mu} k_{3 \nu} k_{\lambda}+k_{3 \lambda} g_{\mu \nu} k^{2}-k_{3 \mu} g_{\nu \lambda} k^{2}-k_{3 \nu} g_{\mu \lambda} k^{2}-2 k_{\lambda} g_{\mu \nu} k \cdot k_{3}\right)+ \\
& +\left(x_{2}-x_{1}\right)\left(2 k_{2 \nu} k_{\lambda} k_{\mu}+2 k_{\nu} k_{2 \lambda} k_{\mu}+k_{2 \mu} g_{\nu \lambda} k^{2}-k_{2 \nu} g_{\lambda \mu} k^{2}-k_{2 \lambda} g_{\nu \mu} k^{2}-2 k_{\mu} g_{\nu \lambda} k \cdot k_{2}\right)+ \\
& +x_{2}\left(2 k_{3 \nu} k_{\lambda} k_{\mu}+2 k_{\nu} k_{3 \lambda} k_{\mu}+k_{3 \mu} g_{\nu \lambda} k^{2}-k_{3 \nu} g_{\lambda \mu} k^{2}-k_{3 \lambda} g_{\nu \mu} k^{2}-2 k_{\mu} g_{\nu \lambda} k \cdot k_{3}\right)+ \\
& +\left(x_{2}-x_{1}\right)\left(2 k_{2 \lambda} k_{\mu} k_{\nu}+2 k_{\lambda} k_{2 \mu} k_{\nu}+k_{2 \nu} g_{\lambda \mu} k^{2}-k_{2 \lambda} g_{\mu \nu} k^{2}-k_{2 \mu} g_{\lambda \nu} k^{2}-2 k_{\nu} g_{\lambda \mu} k \cdot k_{2}\right)+ \\
& \left.+\left(x_{2}-1\right)\left(2 k_{3 \lambda} k_{\mu} k_{\nu}+2 k_{\lambda} k_{3 \mu} k_{\nu}+k_{3 \nu} g_{\lambda \mu} k^{2}-k_{3 \lambda} g_{\mu \nu} k^{2}-k_{3 \mu} g_{\lambda \nu} k^{2}-2 k_{\nu} g_{\lambda \mu} k \cdot k_{3}\right)\right]
\end{aligned}
$$

with the relation $I_{0 \mu \nu}^{R}=\frac{1}{4} g_{\mu \nu} I_{0}^{R}$, all the divergent parts can be expressed in term of $I_{0}^{R}$. The result is given by

$$
\begin{aligned}
L(6 a)_{\mu \nu \lambda ; d i v}^{a b c R}= & 2 i g^{3} f^{a b c} T_{2} \int_{0}^{1} d x_{1} \int_{0}^{x_{1}} d x_{2} I_{0}^{R}\left[4 ( - x _ { 1 } + x _ { 2 } + 1 ) \left(\frac{1}{2} k_{2 \mu} g_{\nu \lambda}+\right.\right. \\
& \left.+\frac{1}{2} k_{2 \nu} g_{\mu \lambda}+k_{2 \lambda} g_{\mu \nu}-k_{2 \mu} g_{\nu \lambda}-k_{2 \nu} g_{\mu \lambda}-\frac{1}{2} k_{2 \lambda} g_{\mu \nu}\right)+x_{2}\left(\frac{1}{2} k_{3 \mu} g_{\nu \lambda}+\right. \\
& \left.+\frac{1}{2} k_{3 \nu} g_{\mu \lambda}+k_{3 \lambda} g_{\mu \nu}-k_{3 \mu} g_{\nu \lambda}-k_{3 \nu} g_{\mu \lambda}-\frac{1}{2} k_{3 \lambda} g_{\mu \nu}\right)+ \\
& +\left(x_{2}-x_{1}\right)\left(\frac{1}{2} k_{2 \nu} g_{\lambda \mu}+\frac{1}{2} k_{2 \lambda} g_{\nu \mu}+k_{2 \mu} g_{\nu \lambda}-k_{2 \nu} g_{\lambda \mu}-k_{2 \lambda} g_{\nu \mu}-\frac{1}{2} k_{2 \mu} g_{\nu \lambda}\right)+ \\
& +x_{2}\left(\frac{1}{2} k_{3 \nu} g_{\lambda \mu}+\frac{1}{2} k_{3 \lambda} g_{\nu \mu}+k_{3 \mu} g_{\nu \lambda}-k_{3 \nu} g_{\lambda \mu}-k_{3 \lambda} g_{\nu \mu}-\frac{1}{2} k_{3 \mu} g_{\nu \lambda}\right)+ \\
& +\left(x_{2}-x_{1}\right)\left(\frac{1}{2} k_{2 \lambda} g_{\mu \nu}+\frac{1}{2} k_{2 \mu} g_{\lambda \nu}+k_{2 \nu} g_{\lambda \mu}-k_{2 \lambda} g_{\mu \nu}-k_{2 \mu} g_{\lambda \nu}-\frac{1}{2} k_{\nu} g_{\lambda \mu}\right)+ \\
& \left.+\left(x_{2}-1\right)\left(\frac{1}{2} k_{3 \lambda} g_{\mu \nu}+\frac{1}{2} k_{3 \mu} g_{\lambda \nu}+k_{3 \nu} g_{\lambda \mu}-k_{3 \lambda} g_{\mu \nu}-k_{3 \mu} g_{\lambda \nu}-\frac{1}{2} k_{3 \nu} g_{\lambda \mu}\right)\right]
\end{aligned}
$$

The second diagram (Fig.6b) has the following form 


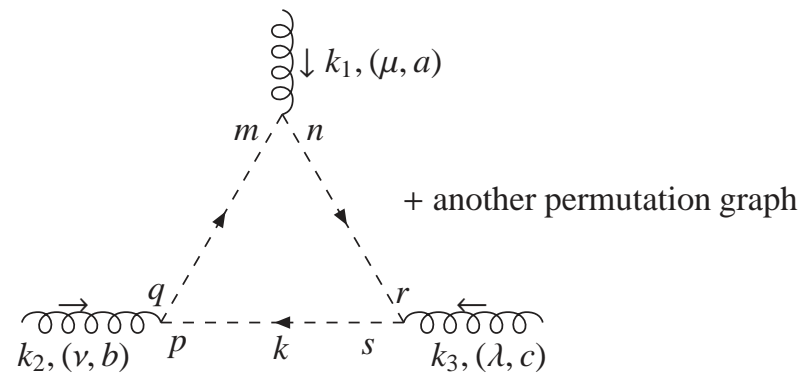

Fig.6b.

$$
\begin{aligned}
L(6 b)_{\mu \nu \lambda}^{a b c}= & -\int \frac{d^{4} k}{(2 \pi)^{4}} g f^{a n m}\left(k-k_{3}\right)_{\mu} \frac{i \delta^{m q}}{\left(k+k_{2}\right)^{2}} g f^{b q p}\left(k+k_{2}\right)_{\nu} \frac{i \delta^{p s}}{k^{2}} g f^{c s r} k_{\lambda} \frac{i \delta^{r n}}{\left(k-k_{3}\right)^{2}} \\
& -\int \frac{d^{4} k}{(2 \pi)^{4}} g f^{a n m}\left(k-k_{2}\right)_{\mu} \frac{i \delta^{m q}}{\left(k+k_{3}\right)^{2}} g f^{c q p}\left(k+k_{3}\right)_{\lambda} \frac{i \delta^{p s}}{k^{2}} g f^{b s r} k_{\nu} \frac{i \delta^{r n}}{\left(k-k_{2}\right)^{2}} \\
= & i g^{3} f^{a n m} f^{b m p} f^{c p n} \int \frac{d^{4} k}{(2 \pi)^{4}} \frac{\left(k-k_{3}\right)_{\mu}\left(k+k_{2}\right)_{\nu} k_{\lambda}}{\left(k+k_{2}\right)^{2} k^{2}\left(k-k_{3}\right)^{2}}+\left(k_{2} \rightarrow k_{3}, \nu \rightarrow \lambda, b \rightarrow c\right) \\
= & i g^{3} f^{a n m} f^{b m p} f^{c p n} \int_{0}^{1} d x_{1} \int_{0}^{x_{1}} d x_{2} \int \frac{d^{4} k}{(2 \pi)^{4}} \frac{\Gamma(3)}{\Gamma(1)^{3}} \frac{\left(k-k_{3}\right)_{\mu}\left(k+k_{2}\right)_{\nu} k_{\lambda}}{\left[\left(1-x_{1}\right)\left(k+k_{2}\right)^{2}+\left(x_{1}-x_{2}\right) k^{2}+x_{2}\left(k-k_{3}\right)^{2}\right]^{3}} \\
& +\left(k_{2} \rightarrow k_{3}, \nu \rightarrow \lambda, b \rightarrow c\right) \\
= & 2 i g^{3} f^{a n m} f^{b m p} f^{c p n} \int_{0}^{1} d x_{1} \int_{0}^{x_{1}} d x_{2} \int \frac{d^{4} k}{(2 \pi)^{4}} \frac{\left(k+k_{2}\right)_{\nu} k_{\lambda}}{\left\{\left[k+\left(1-x_{1}\right) k_{2}-x_{2} k_{3}\right]^{2}+x_{1}\left(1-x_{1}\right) k_{2}^{2}+x_{2}\left(1-x_{2}\right) k_{3}^{2}\right\}^{3}} \\
& +\left(k_{2} \rightarrow k_{3}, \nu \rightarrow \lambda, b \rightarrow c\right) \\
= & 2 i g^{3} f^{a n m} f^{b m p} f^{c p n} \int_{0}^{1} d x_{1} \int_{0}^{x_{1}} d x_{2} \\
& \int \frac{d^{4} k}{(2 \pi)^{4}} \frac{\left[k-\left(1-x_{1}\right) k_{2}-\left(1-x_{2}\right) k_{3}\right]_{\mu}\left(k+x_{1} k_{2}+x_{2} k_{3}\right)_{\nu}\left[k-\left(1-x_{1}\right) k_{2}+x_{2} k_{3}\right]_{\lambda}}{\left(k^{2}-M_{6 b}^{2}\right)^{3}} \\
& +\left(k_{2} \rightarrow k_{3}, \nu \rightarrow \lambda, b \rightarrow c\right)
\end{aligned}
$$

with $M_{6 b}^{2}=-x_{1}\left(1-x_{1}\right) k_{2}^{2}-x_{2}\left(1-x_{2}\right) k_{3}^{2}$. The divergent part is found to be

$$
\begin{aligned}
L(6 b)_{\mu \nu \lambda ; d i v}^{a b c}= & 2 i g^{3} f^{a n m} f^{b m p} f^{c p n} \int_{0}^{1} d x_{1} \int_{0}^{x_{1}} d x_{2} \\
& \int \frac{d^{4} k}{(2 \pi)^{4}} \frac{k \mu k_{\nu}\left[-\left(1-x_{1}\right) k_{2}+x_{2} k_{3}\right]_{\lambda}+k_{\mu} k_{\lambda}\left(x_{1} k_{2}+x_{2} k_{3}\right)_{\nu}+k_{\nu} k_{\lambda}\left[-\left(1-x_{1}\right) k_{2}-\left(1-x_{2}\right) k_{3}\right]_{\mu}}{\left(k^{2}-M_{6 b}^{2}\right)^{3}} \\
& +\left(k_{2} \rightarrow k_{3}, \nu \rightarrow \lambda, b \rightarrow c\right) \\
= & 2 i g^{3} f^{a n m} f^{b m p} f^{c p n} \int_{0}^{1} d x_{1} \int_{0}^{x_{1}} d x_{2}\left\{\left[-\left(1-x_{1}\right) k_{2}+x_{2} k_{3}\right]_{\lambda} I_{0 \mu \nu}\left(M_{6 b}^{2}\right)+\left(x_{1} k_{2}+x_{2} k_{3}\right)_{\nu} I_{0 \mu \lambda}\left(M_{6 b}^{2}\right)\right. \\
& \left.+\left[-\left(1-x_{1}\right) k_{2}-\left(1-x_{2}\right) k_{3}\right]_{\mu} I_{0 \nu \lambda}\left(M_{6 b}^{2}\right)\right\}+\left(k_{2} \rightarrow k_{3}, \nu \rightarrow \lambda, b \rightarrow c\right)
\end{aligned}
$$

Taking the relation $I_{0 \mu \nu}^{R}=\frac{1}{4} g_{\mu \nu} I_{0}^{R}$, we arrive at the result

$$
\begin{aligned}
L(6 b)_{\mu \nu \lambda ; d i v}^{a b c R}= & 2 i g^{3} f^{a n m} f^{b m p} f^{c p n} \int_{0}^{1} d x_{1} \int_{0}^{x_{1}} d x_{2} \\
& \left\{\left[-\left(1-x_{1}\right) k_{2}+x_{2} k_{3}\right]_{\lambda} \frac{1}{4} g_{\mu \nu}+\left(x_{1} k_{2}+x_{2} k_{3}\right)_{\nu} \frac{1}{4} g_{\mu \lambda}+\left[-\left(1-x_{1}\right) k_{2}-\left(1-x_{2}\right) k_{3}\right]_{\mu} \frac{1}{4} g_{\nu \lambda}\right\} I_{0}^{R}\left(M_{6 b}^{2}\right) \\
& +\left(k_{2} \rightarrow k_{3}, \nu \rightarrow \lambda, b \rightarrow c\right)
\end{aligned}
$$

The third diagram (Fig.6c) is given by 


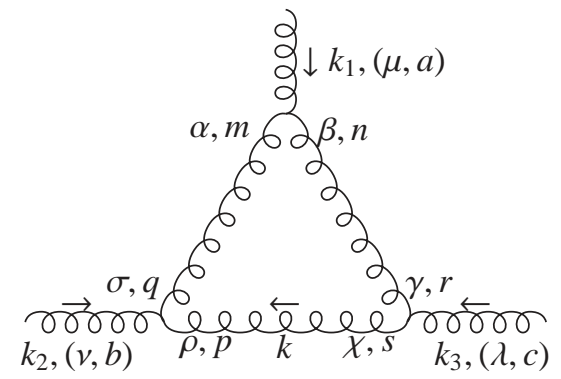

Fig.6c.

$$
\begin{aligned}
& L(6 c)_{\mu \nu \lambda}^{a b c}=\int \frac{d^{4} k}{(2 \pi)^{4}} g f^{a m n}\left[\left(k_{1}-k-k_{2}\right)_{\beta} g_{\mu \alpha}+\left(k+k_{2}-k_{3}+k\right)_{\mu} g_{\alpha \beta}+\left(k_{3}-k-k_{1}\right)_{\alpha} g_{\beta \mu}\right] \frac{-i \delta^{q m} g^{\sigma \alpha}}{\left(k+k_{2}\right)^{2}} \\
& \times g f^{b p q}\left[\left(k_{2}-k\right)_{\sigma} g_{\nu \rho}+\left(k+k+k_{2}\right)_{\nu} g_{\rho \sigma}+\left(-k-k_{2}-k_{2}\right)_{\rho} g_{\sigma \nu}\right] \frac{-i \delta^{p s} g^{\rho \chi}}{k^{2}} \\
& \times g f^{c r s}\left[\left(k_{3}-k+k_{3}\right)_{\chi} g_{\lambda \gamma}+\left(k-k_{3}+k\right)_{\lambda} g_{\gamma \chi}+\left(-k-k_{3}\right)_{\gamma} g_{\chi \lambda}\right] \frac{-i \delta^{r n} g^{\gamma \beta}}{\left(k-k_{3}\right)^{2}} \\
& =(-i g)^{3} f^{a m n} f^{b p m} f^{c n p} \int \frac{d^{4} k}{(2 \pi)^{4}}\left[\left(k_{1}-k-k_{2}\right)^{\gamma} g_{\mu \alpha}+\left(k+k_{2}-k_{3}+k\right)_{\mu} g_{\alpha}^{\gamma}+\left(k_{3}-k-k_{1}\right)_{\alpha} g_{\mu}^{\gamma}\right] \\
& \times\left[\left(k_{2}-k\right)^{\alpha} g_{\nu \rho}+\left(k+k+k_{2}\right)_{\nu} g_{\rho}^{\alpha}+\left(-k-k_{2}-k_{2}\right)_{\rho} g_{\nu}^{\alpha}\right] \\
& \times\left[\left(k_{3}-k+k_{3}\right)^{\rho} g_{\lambda \gamma}+\left(k-k_{3}+k\right)_{\lambda} g_{\gamma}^{\rho}+\left(-k-k_{3}\right)_{\gamma} g_{\lambda}^{\rho}\right] \\
& \times \frac{1}{\left(k+k_{2}\right)^{2} k^{2}\left(k-k_{3}\right)^{2}} \\
& =(-i g)^{3}\left(-\frac{1}{2} C_{1} f^{a b c}\right) \int_{0}^{1} d x_{1} \int_{0}^{x_{1}} d x_{2} \int \frac{d^{4} k}{(2 \pi)^{4}} \frac{\Gamma(3)}{\Gamma(1)^{3}} \frac{1}{\left[\left(1-x_{1}\right)\left(k+k_{2}\right)^{2}+\left(x_{1}-x_{2}\right) k^{2}+x_{2}\left(k-k_{3}\right)^{2}\right]^{3}} \\
& \times\left[\left(k_{1}-k-k_{2}\right)^{\gamma} g_{\mu \alpha}+\left(k+k_{2}-k_{3}+k\right)_{\mu} g_{\alpha}^{\gamma}+\left(k_{3}-k-k_{1}\right)_{\alpha} g_{\mu}^{\gamma}\right] \\
& \times\left[\left(k_{2}-k\right)^{\alpha} g_{\nu \rho}+\left(k+k+k_{2}\right)_{\nu} g_{\rho}^{\alpha}+\left(-k-k_{2}-k_{2}\right)_{\rho} g_{\nu}^{\alpha}\right] \\
& \times\left[\left(k_{3}-k+k_{3}\right)^{\rho} g_{\lambda \gamma}+\left(k-k_{3}+k\right)_{\lambda} g_{\gamma}^{\rho}+\left(-k-k_{3}\right)_{\gamma} g_{\lambda}^{\rho}\right] \\
& =-i C_{1} g^{3} f^{a b c} \int_{0}^{1} d x_{1} \int_{0}^{x_{1}} d x_{2} \int \frac{d^{4} k}{(2 \pi)^{4}} \frac{1}{\left\{\left[k+\left(1-x_{1}\right) k_{2}-x_{2} k_{3}\right]^{2}+x_{1}\left(1-x_{1}\right) k_{2}^{2}+x_{2}\left(1-x_{2}\right) k_{3}^{2}\right\}^{3}} \\
& \times\left[\left(k_{1}-k-k_{2}\right)^{\gamma} g_{\mu \alpha}+\left(k+k_{2}-k_{3}+k\right)_{\mu} g_{\alpha}^{\gamma}+\left(k_{3}-k-k_{1}\right)_{\alpha} g_{\mu}^{\gamma}\right] \\
& \times\left[\left(k_{2}-k\right)^{\alpha} g_{\nu \rho}+\left(k+k+k_{2}\right)_{\nu} g_{\rho}^{\alpha}+\left(-k-k_{2}-k_{2}\right)_{\rho} g_{\nu}^{\alpha}\right] \times \\
& {\left[\left(k_{3}-k+k_{3}\right)^{\rho} g_{\lambda \gamma}+\left(k-k_{3}+k\right)_{\lambda} g_{\gamma}^{\rho}+\left(-k-k_{3}\right)_{\gamma} g_{\lambda}^{\rho}\right]} \\
& =-i C_{1} g^{3} f^{a b c} \int_{0}^{1} d x_{1} \int_{0}^{x_{1}} d x_{2} \int \frac{d^{4} k}{(2 \pi)^{4}} \frac{1}{\left(k^{2}-M_{6 c}^{2}\right)^{3}} \\
& \times\left[\left(-k+\left(-1-x_{1}\right) k_{2}+\left(-1-x_{2}\right) k_{3}\right)^{\gamma} g_{\mu \alpha}+\left(2 k+\left(-1+2 x_{1}\right) k_{2}+\left(-1+2 x_{2}\right) k_{3}\right){ }_{\mu} g_{\alpha}^{\gamma}+\right. \\
& \left.\left(-k+\left(2-x_{1}\right) k_{2}+\left(2-x_{2}\right) k_{3}\right)_{\alpha} g_{\mu}^{\gamma}\right] \\
& \times\left[\left(-k+\left(2-x_{1}\right) k_{2}-x_{2} k_{3}\right)^{\alpha} g_{\nu \rho}+\left(2 k+\left(-1+2 x_{1}\right) k_{2}+2 x_{2} k_{3}\right)_{\nu} g_{\rho}^{\alpha}+\right. \\
& \left.\left(-k+\left(-1-x_{1}\right) k_{2}-x_{2} k_{3}\right)_{\rho} g_{\nu}^{\alpha}\right] \\
& \times\left[\left(-k+\left(1-x_{1}\right) k_{2}+\left(2-x_{2}\right) k_{3}\right)^{\rho} g_{\lambda \gamma}+\left(2 k+\left(-2+2 x_{1}\right) k_{2}+\left(-1+2 x_{2}\right) k_{3}\right)_{\lambda} g_{\gamma}^{\rho}+\right. \\
& \left.\left(-k+\left(1-x_{1}\right) k_{2}+\left(-1-x_{2}\right) k_{3}\right)_{\gamma} g_{\lambda}^{\rho}\right]
\end{aligned}
$$

with $M_{6 c}^{2}=-x_{1}\left(1-x_{1}\right) k_{2}^{2}-x_{2}\left(1-x_{2}\right) k_{3}^{2}$. The divergent part reads:

$$
L(6 c)_{\mu \nu \lambda ; d i v}^{a b c}=-i C_{1} g^{3} f^{a b c} \int_{0}^{1} d x_{1} \int_{0}^{x_{1}} d x_{2} \int \frac{d^{4} k}{(2 \pi)^{4}} \frac{1}{\left(k^{2}-M_{6 c}^{2}\right)^{3}} \times
$$




$$
\begin{aligned}
& \left\{\left[-k^{\alpha} g_{\nu \rho}+2 k_{\nu} g_{\rho}^{\alpha}-k_{\rho} g_{\nu}^{\alpha}\right]\left[-k^{\rho} g_{\lambda \gamma}+2 k_{\lambda} g_{\gamma}^{\rho}-k_{\gamma} g_{\lambda}^{\rho}\right] \times\left[\left(\left(-1-x_{1}\right) k_{2}+\left(-1-x_{2}\right) k_{3}\right)^{\gamma} g_{\mu \alpha}\right.\right. \\
& \left.+\left(\left(-1+2 x_{1}\right) k_{2}+\left(-1+2 x_{2}\right) k_{3}\right)_{\mu} g_{\alpha}^{\gamma}+\left(\left(2-x_{1}\right) k_{2}+\left(2-x_{2}\right) k_{3}\right)_{\alpha} g_{\mu}^{\gamma}\right]+ \\
& {\left[-k^{\gamma} g_{\mu \alpha}+2 k_{\mu} g_{\alpha}^{\gamma}-k_{\alpha} g_{\mu}^{\gamma}\right]\left[-k^{\rho} g_{\lambda \gamma}+2 k_{\lambda} g_{\gamma}^{\rho}-k_{\gamma} g_{\lambda}^{\rho}\right] \times } \\
& {\left[\left(\left(2-x_{1}\right) k_{2}-x_{2} k_{3}\right)^{\alpha} g_{\nu \rho}+\left(\left(-1+2 x_{1}\right) k_{2}+2 x_{2} k_{3}\right)_{\nu} g_{\rho}^{\alpha}+\left(\left(-1-x_{1}\right) k_{2}-x_{2} k_{3}\right)_{\rho} g_{\nu}^{\alpha}\right]+} \\
& {\left[-k^{\gamma} g_{\mu \alpha}+2 k_{\mu} g_{\alpha}^{\gamma}-k_{\alpha} g_{\mu}^{\gamma}\right]\left[-k^{\alpha} g_{\nu \rho}+2 k_{\nu} g_{\rho}^{\alpha}-k_{\rho} g_{\nu}^{\alpha}\right] \times\left[\left(\left(1-x_{1}\right) k_{2}+\left(2-x_{2}\right) k_{3}\right)^{\rho} g_{\lambda \gamma}\right.} \\
& \left.\left.+\left(\left(-2+2 x_{1}\right) k_{2}+\left(-1+2 x_{2}\right) k_{3}\right)_{\lambda} g_{\gamma}^{\rho}+\left(\left(1-x_{1}\right) k_{2}+\left(-1-x_{2}\right) k_{3}\right)_{\gamma} g_{\lambda}^{\rho}\right]\right\} \\
= & -i C_{1} g^{3} f^{a b c} \int_{0}^{1} d x_{1} \int_{0}^{x_{1}} d x_{2} \int \frac{d^{4} k}{(2 \pi)^{4}} \frac{1}{\left(k^{2}-M_{6 c}^{2}\right)^{3}} \times \\
& \left\{\left(k^{\alpha} k_{\gamma} g_{\lambda \nu}-2 k^{\alpha} k_{\lambda} g_{\nu \gamma}-k_{\gamma} k_{\lambda} g_{\nu}^{\alpha}-k^{\alpha} k_{\nu} g_{\lambda \gamma}-2 k_{\nu} k_{\gamma} g_{\lambda}^{\alpha}+4 k_{\nu} k_{\lambda} g_{\gamma}^{\alpha}+k^{2} g_{\nu}^{\alpha} g_{\gamma \lambda}\right) \times\left[\left(-1-x_{1}\right) k_{2}\right.\right. \\
& \left.\left.+\left(-1-x_{2}\right) k_{3}\right)^{\gamma} g_{\mu \alpha}+\left(\left(-1+2 x_{1}\right) k_{2}+\left(-1+2 x_{2}\right) k_{3}\right)_{\mu} g_{\alpha}^{\gamma}+\left(\left(2-x_{1}\right) k_{2}+\left(2-x_{2}\right) k_{3}\right)_{\alpha} g_{\mu}^{\gamma}\right]+ \\
& \left(-2 k_{\alpha} k_{\lambda} g_{\mu}^{\rho}-k_{\alpha} k_{\mu} g_{\lambda}^{\rho}+4 k_{\mu} k_{\lambda} g_{\alpha}^{\rho}+k^{\rho} k_{\alpha} g_{\mu \lambda}-k^{\rho} k_{\lambda} g_{\mu \alpha}-2 k^{\rho} k_{\mu} g_{\alpha \lambda}+k^{2} g_{\lambda}^{\rho} g_{\alpha \mu}\right) \times \\
& {\left[\left(\left(2-x_{1}\right) k_{2}-x_{2} k_{3}\right)^{\alpha} g_{\nu \rho}+\left(\left(-1+2 x_{1}\right) k_{2}+2 x_{2} k_{3}\right)_{\nu} g_{\rho}^{\alpha}+\left(\left(-1-x_{1}\right) k_{2}-x_{2} k_{3}\right)_{\rho} g_{\nu}^{\alpha}\right]+} \\
& \left(-k^{\gamma} k_{\mu} g_{\nu \rho}+k^{2} g_{\mu}^{\gamma} g_{\nu \rho}-2 k^{\gamma} k_{\nu} g \mu \rho+4 k_{\mu} k_{\nu} g_{\rho}^{\gamma}+k^{\gamma} k_{\rho} g_{\mu \nu}-2 k_{\mu} k_{\rho} g_{\nu}^{\gamma}-k_{\nu} k_{\rho} g_{\mu}^{\gamma}\right) \times\left[\left(\left(1-x_{1}\right) k_{2}\right.\right. \\
& \left.\left.\left.+\left(2-x_{2}\right) k_{3}\right)^{\rho} g_{\lambda \gamma}+\left(\left(-2+2 x_{1}\right) k_{2}+\left(-1+2 x_{2}\right) k_{3}\right)_{\lambda} g_{\gamma}^{\rho}+\left(\left(1-x_{1}\right) k_{2}+\left(-1-x_{2}\right) k_{3}\right)_{\gamma} g_{\lambda}^{\rho}\right]\right\} \quad(\mathrm{C} 27)
\end{aligned}
$$

After adopting the relation $I_{0 \mu \nu}^{R}=\frac{1}{4} g_{\mu \nu} I_{0}^{R}$, we arrive at the following result:

$$
\begin{aligned}
L(6 c)_{\mu \nu \lambda ; d i v}^{a b c R} & =-i C_{1} g^{3} f^{a b c} \int_{0}^{1} d x_{1} \int_{0}^{x_{1}} d x_{2} I_{0}^{R}\left(M_{6 c}^{2}\right) \times \\
& \left\{\left(\frac{1}{4} g^{\alpha} \gamma g_{\lambda \nu}-\frac{2}{4} g_{\lambda}^{\alpha} g_{\nu \gamma}-\frac{1}{4} g_{\gamma \lambda} g_{\nu}^{\alpha}-\frac{1}{4} g_{\nu}^{\alpha} g_{\lambda \gamma}-\frac{2}{4} g_{\nu \gamma} g_{\lambda}^{\alpha}+\frac{4}{4} g_{\nu \lambda} g_{\gamma}^{\alpha}+g_{\nu}^{\alpha} g_{\gamma \lambda}\right) \times\left[\left(\left(-1-x_{1}\right) k_{2}\right.\right.\right. \\
& \left.\left.+\left(-1-x_{2}\right) k_{3}\right)^{\gamma} g_{\mu \alpha}+\left(\left(-1+2 x_{1}\right) k_{2}+\left(-1+2 x_{2}\right) k_{3}\right)_{\mu} g_{\alpha}^{\gamma}+\left(\left(2-x_{1}\right) k_{2}+\left(2-x_{2}\right) k_{3}\right)_{\alpha} g_{\mu}^{\gamma}\right]+ \\
& \left(-\frac{2}{4} g_{\alpha \lambda} g_{\mu}^{\rho}-\frac{1}{4} g_{\alpha \mu} g_{\lambda}^{\rho}+\frac{4}{4} g_{\mu \lambda} g_{\alpha}^{\rho}+\frac{1}{4} g_{\alpha}^{\rho} g_{\mu \lambda}-\frac{1}{4} g_{\lambda}^{\rho} g_{\mu \alpha}-\frac{2}{4} g_{\mu}^{\rho} g_{\alpha \lambda}+g_{\lambda}^{\rho} g_{\alpha \mu}\right) \times \\
& {\left[\left(\left(2-x_{1}\right) k_{2}-x_{2} k_{3}\right)^{\alpha} g_{\nu \rho}+\left(\left(-1+2 x_{1}\right) k_{2}+2 x_{2} k_{3}\right)_{\nu} g_{\rho}^{\alpha}+\left(\left(-1-x_{1}\right) k_{2}-x_{2} k_{3}\right)_{\rho} g_{\nu}^{\alpha}\right]+} \\
& \left(-\frac{1}{4} g_{\mu}^{\gamma} g_{\nu \rho}+g_{\mu}^{\gamma} g_{\nu \rho}-\frac{2}{4} g_{\nu}^{\gamma} g \mu \rho+\frac{4}{4} g_{\mu \nu} g_{\rho}^{\gamma}+\frac{1}{4} g_{\rho}^{\gamma} g_{\mu \nu}-\frac{2}{4} g_{\mu \rho} g_{\nu}^{\gamma}-\frac{1}{4} g_{\nu \rho} g_{\mu}^{\gamma}\right) \times\left[\left(\left(1-x_{1}\right) k_{2}\right.\right. \\
& \left.\left.\left.+\left(2-x_{2}\right) k_{3}\right)^{\rho} g_{\lambda \gamma}+\left(\left(-2+2 x_{1}\right) k_{2}+\left(-1+2 x_{2}\right) k_{3}\right)_{\lambda} g_{\gamma}^{\rho}+\left(\left(1-x_{1}\right) k_{2}+\left(-1-x_{2}\right) k_{3}\right)_{\gamma} g_{\lambda}^{\rho}\right]\right\}
\end{aligned}
$$

Similarly, the fourth diagram (Fig.6d) is given:

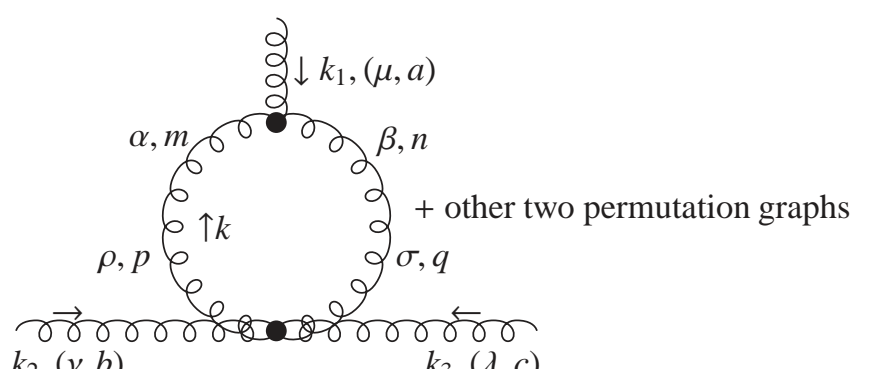

Fig.6d.

$$
\begin{aligned}
L(6 d)_{\mu \nu \lambda}^{a b c}= & \frac{1}{2 !} \int \frac{d^{4} k}{(2 \pi)^{4}} g f^{a m n}\left[\left(k_{1}-k\right)_{\beta} g_{\mu \alpha}+\left(k+k+k_{1}\right)_{\mu} g_{\alpha \beta}+\left(-k-k_{1}-k_{1}\right)_{\alpha} g_{\beta \mu}\right] \frac{-i \delta^{m p} g^{\alpha \rho}}{k^{2}} \frac{-i \delta^{n q} g^{\beta \sigma}}{\left(k+k_{1}\right)^{2}} \\
& \times\left(-i g^{2}\right)\left[f^{e p q} f^{e c b}\left(g_{\rho \lambda} g_{\sigma \nu}-g_{\nu \rho} g_{\lambda \sigma}\right)+f^{e p c} f^{e b q}\left(g_{\nu \rho} g_{\lambda \sigma}-g_{\rho \sigma} g_{\nu \lambda}\right)+f^{e p b} f^{e q c}\left(g_{\rho \sigma} g_{\nu \lambda}-g_{\rho \lambda} g_{\nu \sigma}\right)\right] \\
& + \text { permutation graphs } \\
= & \frac{1}{2} i g^{3} f^{a p q}\left[f^{e p q} f^{e c b}\left(g_{\rho \lambda} g_{\sigma \nu}-g_{\nu \rho} g_{\lambda \sigma}\right)+f^{e p c} f^{e b q}\left(g_{\nu \rho} g_{\lambda \sigma}-g_{\rho \sigma} g_{\nu \lambda}\right)+f^{e p b} f^{e q c}\left(g_{\rho \sigma} g_{\nu \lambda}-g_{\rho \lambda} g_{\nu \sigma}\right)\right] \\
& \times \int \frac{d^{4} k}{(2 \pi)^{4}}\left[\left(k_{1}-k\right)^{\sigma} g_{\mu}^{\rho}+\left(k+k+k_{1}\right)_{\mu} g^{\rho \sigma}+\left(-k-k_{1}-k_{1}\right)^{\rho} g_{\mu}^{\sigma}\right] \frac{1}{k^{2}\left(k+k_{1}\right)^{2}}
\end{aligned}
$$




$$
\begin{aligned}
& + \text { permutation graphs } \\
= & \frac{i}{2} g^{3} C_{1} f^{a b c}\left[-\left(g_{\rho \lambda} g_{\sigma \nu}-g_{\nu \rho} g_{\lambda \sigma}\right)+\frac{1}{2}\left(g_{\nu \rho} g_{\lambda \sigma}-g_{\rho \sigma} g_{\nu \lambda}\right)+\frac{1}{2}\left(g_{\rho \sigma} g_{\nu \lambda}-g_{\rho \lambda} g_{\nu \sigma}\right)\right] \int_{0}^{1} d x_{1} \times \\
& \int \frac{d^{4} k}{(2 \pi)^{4}} \frac{\Gamma(2)}{\Gamma(1)^{2}} \frac{1}{\left[\left(1-x_{1}\right) k^{2}+x_{1}\left(k+k_{1}\right)^{2}\right]^{2}}\left[\left(k_{1}-k\right)^{\sigma} g_{\mu}^{\rho}+\left(k+k+k_{1}\right)_{\mu} g^{\rho \sigma}+\left(-k-k_{1}-k_{1}\right)^{\rho} g_{\mu}^{\sigma}\right] \\
& + \text { permutation graphs } \\
= & \frac{i}{2} g^{3} C_{1} f^{a b c} \frac{3}{2}\left(g_{\nu \rho} g_{\lambda \sigma}-g_{\rho \lambda} g_{\sigma \nu}\right) \int_{0}^{1} d x_{1} \int \frac{d^{4} k}{(2 \pi)^{4}} \frac{1}{\left[\left(k+x_{1} k_{1}\right)^{2}+x_{1}\left(1-x_{1}\right) k_{1}^{2}\right]^{2}} \times \\
& {\left[\left(k_{1}-k\right)^{\sigma} g_{\mu}^{\rho}+\left(k+k+k_{1}\right)_{\mu} g^{\rho \sigma}+\left(-k-k_{1}-k_{1}\right)^{\rho} g_{\mu}^{\sigma}\right]+\text { permutation graphs } } \\
= & \frac{3 i}{4} g^{3} C_{1} f^{a b c}\left(g_{\nu \rho} g_{\lambda \sigma}-g_{\rho \lambda} g_{\sigma \nu}\right) \int_{0}^{1} d x_{1} \int \frac{d^{4} k}{(2 \pi)^{4}} \frac{1}{\left(k^{2}-M_{6 d}^{2}\right)^{2}}\left[\left(-k+\left(1+x_{1}\right) k_{1}\right)^{\sigma} g_{\mu}^{\rho}\right. \\
& \left.+\left(2 k+\left(1-2 x_{1}\right) k_{1}\right)_{\mu} g^{\rho \sigma}+\left(-k+\left(-2+x_{1}\right) k_{1}\right)^{\rho} g_{\mu}^{\sigma}\right]+ \text { permutation graphs }
\end{aligned}
$$

with $M_{6 d}^{2}=-x_{1}\left(1-x_{1}\right) k_{1}^{2}$. The finally result is simply given by

$$
\begin{aligned}
L(6 d)_{\mu \nu \lambda ; d i v}^{a b c R}= & \frac{3 i}{4} g^{3} C_{1} f^{a b c}\left(g_{\nu \rho} g_{\lambda \sigma}-g_{\rho \lambda} g_{\sigma \nu}\right) \int_{0}^{1} d x_{1} I_{0}\left(M_{6 d}^{2}\right)\left[\left(1+x_{1}\right) k_{1}^{\sigma} g_{\mu}^{\rho}+\left(1-2 x_{1}\right) k_{1 \mu} g^{\rho \sigma}+\left(-2+x_{1}\right) k_{1}^{\rho} g_{\mu}^{\sigma}\right] \\
& + \text { permutation graphs }
\end{aligned}
$$

\section{7. four-gluon vertex renormalization} by:

There are five diagrams which can contribute to four-gluon vertex renormalizaion. The first one (Fig.7a) is given

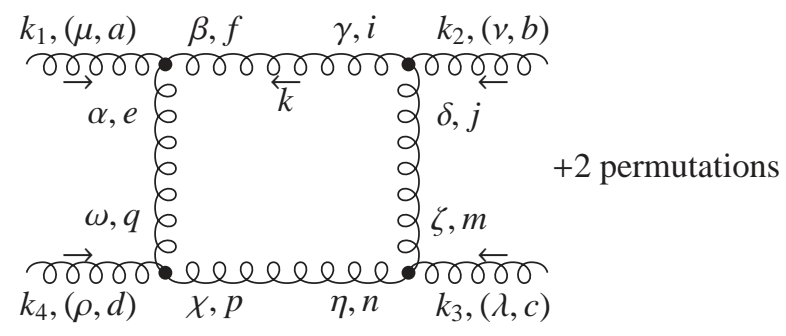

Fig.7a.

$$
\begin{aligned}
L(7 a)_{\mu \nu \lambda \rho}^{a b c d}= & \int \frac{d^{4} k}{(2 \pi)^{4}} g f^{a e f}\left[\left(k_{1}+k+k_{1}\right)_{\beta} g_{\mu \alpha}+\left(-k-k_{1}-k\right)_{\mu} g_{\alpha \beta}+\left(k-k_{1}\right)_{\alpha} g_{\beta \mu}\right] \frac{-i \delta^{f i} g^{\beta \gamma}}{k^{2}} \times \\
& g f^{b i j}\left[\left(k_{2}+k\right)_{\delta} g_{\nu \gamma}+\left(-k-k+k_{2}\right)_{\nu} g_{\gamma \delta}+\left(k-k_{2}-k_{2}\right)_{\gamma} g_{\delta \nu}\right] \frac{-i \delta^{j m} g^{\delta \zeta}}{\left(k-k_{2}\right)^{2}} \times \\
& g f^{c m n}\left[\left(k_{3}-k_{2}+k\right)_{\eta} g_{\lambda \zeta}+\left(k_{2}-k-k+k_{2}+k_{3}\right)_{\lambda} g_{\zeta \eta}+\left(k-k_{2}-k_{3}-k_{3}\right)_{\zeta} g_{\eta \lambda}\right] \frac{-i \delta^{p n} g^{\eta \chi}}{\left(k-k_{2}-k_{3}\right)^{2}} \times \\
& f^{d p q}\left[\left(k_{4}+k+k_{1}+k_{4}\right)_{\omega} g_{\rho \chi}+\left(-k-k_{1}-k_{4}-k-k_{1}\right)_{\rho} g_{\chi \omega}+\left(k+k_{1}-k_{4}\right)_{\chi} g_{\omega \rho}\right] \frac{-i \delta^{q e} g^{\omega \alpha}}{\left(k+k_{1}\right)^{2}} \\
& +2 \text { permutations }
\end{aligned}
$$

its divergent part reads:

$$
L(7 a)_{\mu \nu \lambda \rho ; d i v}^{a b c d}=\int \frac{d^{4} k}{(2 \pi)^{4}} g f^{a e f}\left(k_{\beta} g_{\mu \alpha}-2 k_{\mu} g_{\alpha \beta}+k_{\alpha} g_{\beta \mu}\right) \frac{-i \delta^{f i} g^{\beta \gamma}}{k^{2}} g f^{b i j}\left(k_{\delta} g_{\nu \gamma}-2 k_{\nu} g_{\gamma \delta}+k_{\gamma} g_{\delta \nu}\right) \frac{-i \delta^{j m} g^{\delta \zeta}}{\left(k-k_{2}\right)^{2}} \times
$$




$$
\begin{aligned}
& g f^{c m n}\left(k_{\eta} g_{\lambda \zeta}-2 k_{\lambda} g_{\zeta \eta}+k_{\zeta} g_{\eta \lambda}\right) \frac{-i \delta^{p n} g^{\eta \chi}}{\left(k-k_{2}-k_{3}\right)^{2}} f^{d p q}\left(k_{\omega} g_{\rho \chi}-2 k_{\rho} g_{\chi \omega}+k_{\chi} g_{\omega \rho}\right) \frac{-i \delta^{q e} g^{\omega \alpha}}{\left(k+k_{1}\right)^{2}} \\
& +2 \text { permutations } \\
& =(-i g)^{4} f^{a e f} f^{b f j} f^{c j n} f^{d n e} \int \frac{d^{4} k}{(2 \pi)^{4}} \times \\
& \frac{\left(k^{\gamma} g_{\mu \alpha}-2 k_{\mu} g_{\alpha}^{\gamma}+k_{\alpha} g_{\mu}^{\gamma}\right)\left(k^{\zeta} g_{\nu \gamma}-2 k_{\nu} g_{\gamma}^{\zeta}+k_{\gamma} g_{\nu}^{\zeta}\right)\left(k^{\chi} g_{\lambda \zeta}-2 k_{\lambda} g_{\zeta}^{\chi}+k_{\zeta} g_{\lambda}^{\chi}\right)\left(k^{\alpha} g_{\rho \chi}-2 k_{\rho} g_{\chi}^{\alpha}+k_{\chi} g_{\rho}^{\alpha}\right)}{k^{2}\left(k-k_{2}\right)^{2}\left(k-k_{2}-k_{3}\right)^{2}\left(k+k_{1}\right)^{2}} \\
& +2 \text { permutations } \\
& =g^{4} f^{a e f} f^{b f j} f^{c j n} f^{d n e} \int_{0}^{1} d x_{1} \int_{0}^{x_{1}} d x_{2} \int_{0}^{x_{2}} d x_{3} \int \frac{d^{4} k}{(2 \pi)^{4}} \frac{\Gamma(4)}{\Gamma(1)^{4}} \times \\
& \frac{\left(k^{\gamma} g_{\mu \alpha}-2 k_{\mu} g_{\alpha}^{\gamma}+k_{\alpha} g_{\mu}^{\gamma}\right)\left(k^{\zeta} g_{\nu \gamma}-2 k_{\nu} g_{\gamma}^{\zeta}+k_{\gamma} g_{\nu}^{\zeta}\right)\left(k^{\chi} g_{\lambda \zeta}-2 k_{\lambda} g_{\zeta}^{\chi}+k_{\zeta} g_{\lambda}^{\chi}\right)\left(k^{\alpha} g_{\rho \chi}-2 k_{\rho} g_{\chi}^{\alpha}+k_{\chi} g_{\rho}^{\alpha}\right)}{\left[\left(1-x_{1}\right) k^{2}+\left(x_{1}-x_{2}\right)\left(k-k_{2}\right)^{2}+\left(x_{2}-x_{3}\right)\left(k-k_{2}-k_{3}\right)^{2}+x_{3}\left(k+k_{1}\right)^{2}\right]^{4}} \\
& +2 \text { permutations } \\
& =6 g^{4} f^{a e f} f^{b f j} f^{c j n} f^{d n e} \int_{0}^{1} d x_{1} \int_{0}^{x_{1}} d x_{2} \int_{0}^{x_{2}} d x_{3} \int \frac{d^{4} k}{(2 \pi)^{4}} \times \\
& \frac{\left(k^{\gamma} g_{\mu \alpha}-2 k_{\mu} g_{\alpha}^{\gamma}+k_{\alpha} g_{\mu}^{\gamma}\right)\left(k^{\zeta} g_{\nu \gamma}-2 k_{\nu} g_{\gamma}^{\zeta}+k_{\gamma} g_{\nu}^{\zeta}\right)\left(k^{\chi} g_{\lambda \zeta}-2 k_{\lambda} g_{\zeta}^{\chi}+k_{\zeta} g_{\lambda}^{\chi}\right)\left(k^{\alpha} g_{\rho \chi}-2 k_{\rho} g_{\chi}^{\alpha}+k_{\chi} g_{\rho}^{\alpha}\right)}{\left\{\left[k-\left(x_{1}-x_{2}\right) k_{2}-\left(x_{2}-x_{3}\right)\left(k_{2}+k_{3}\right)+x_{3} k_{1}\right]^{2}-M_{7 a}^{2}\right\}^{4}} \\
& +2 \text { permutations } \\
& \sim 6 g^{4} f^{a e f} f^{b f j} f^{c j n} f^{d n e} \int_{0}^{1} d x_{1} \int_{0}^{x_{1}} d x_{2} \int_{0}^{x_{2}} d x_{3} \int \frac{d^{4} k}{(2 \pi)^{4}} \frac{1}{\left(k^{2}-M_{7 a}^{2}\right)^{4}} \times \\
& \left(k^{\gamma} g_{\mu \alpha}-2 k_{\mu} g_{\alpha}^{\gamma}+k_{\alpha} g_{\mu}^{\gamma}\right)\left(k^{\zeta} g_{\nu \gamma}-2 k_{\nu} g_{\gamma}^{\zeta}+k_{\gamma} g_{\nu}^{\zeta}\right)\left(k^{\chi} g_{\lambda \zeta}-2 k_{\lambda} g_{\zeta}^{\chi}+k_{\zeta} g_{\lambda}^{\chi}\right)\left(k^{\alpha} g_{\rho \chi}-2 k_{\rho} g_{\chi}^{\alpha}+k_{\chi} g_{\rho}^{\alpha}\right) \\
& +2 \text { permutations } \\
& =6 g^{4} f^{a e f} f^{b f j} f^{c j n} f^{d n e} \int_{0}^{1} d x_{1} \int_{0}^{x_{1}} d x_{2} \int_{0}^{x_{2}} d x_{3} \int \frac{d^{4} k}{(2 \pi)^{4}} \frac{1}{\left(k^{2}-M_{7 a}^{2}\right)^{4}} \times \\
& \left(g_{\mu \nu} g_{\lambda \rho} k^{4}+g_{\mu \rho} g_{\nu \lambda} k^{4}+3 g_{\mu \rho} k_{\nu} k_{\lambda} k^{2}+3 g_{\lambda \rho} k_{\mu} k_{\nu} k^{2}+3 g_{\mu \nu} k_{\rho} k_{\lambda} k^{2}+3 g_{\nu \lambda} k_{\mu} k_{\rho} k^{2}+34 k_{\mu} k_{\nu} k_{\lambda} k_{\rho}\right) \\
& +2 \text { permutations } \\
& \sim 6 g^{4} f^{a e f} f^{b f j} f^{c j n} f^{d n e} \int_{0}^{1} d x_{1} \int_{0}^{x_{1}} d x_{2} \int_{0}^{x_{2}} d x_{3}\left[\left(g_{\mu \nu} g_{\lambda \rho}+g_{\mu \rho} g_{\nu \lambda}\right) I_{0}\left(M_{7 a}^{2}\right)+\right. \\
& \left.3 g_{\mu \rho} I_{0 \nu \lambda}\left(M_{7 a}^{2}\right)+3 g_{\lambda \rho} I_{0 \mu \nu}\left(M_{7 a}^{2}\right)+3 g_{\mu \nu} I_{0 \rho \lambda}\left(M_{7 a}^{2}\right)+3 g_{\nu \lambda} I_{0 \mu \rho}\left(M_{7 a}^{2}\right)+34 I_{0 \mu \nu \lambda \rho}\left(M_{7 a}^{2}\right)\right] \\
& +2 \text { permutations }
\end{aligned}
$$

with

$$
M_{7 a}^{2}=-\left(x_{1}-x_{2}\right)\left(1-x_{1}+x_{2}\right) k_{2}^{2}-\left(x_{2}-x_{3}\right)\left(1-x_{2}+x_{3}\right)\left(k_{2}+k_{3}\right)^{2}-x_{3}\left(1-x_{3}\right) k_{1}^{2} .
$$

and we have used in the last step the identities:

$$
k^{2}=\left(k^{2}-M^{2}\right)+M^{2} \text { and } k^{4}=\left(k^{2}-M^{2}\right)^{2}+2 M^{2}\left(k^{2}-M^{2}\right)+M^{4} .
$$

Taking the relations

$$
I_{0 \mu \nu}^{R}=\frac{1}{4} g_{\mu \nu} I_{0}^{R} \quad I_{0 \mu \nu \rho \sigma}^{R}=\frac{1}{24}\left(g_{\mu \nu} g_{\rho \sigma}+g_{\mu \rho} g_{\nu \sigma}+g_{\mu \sigma} g_{\nu \rho}\right) I_{0}^{R} .
$$

the divergent part can be expressed in term of $I_{0}^{R}$ :

$$
\begin{aligned}
L(7 a)_{\mu \nu \lambda \rho d i v}^{a b c d R}= & 6 g^{4} f^{a e f} f^{b f j} f^{c j n} f^{d n e} \int_{0}^{1} d x_{1} \int_{0}^{x_{1}} d x_{2} \int_{0}^{x_{2}} d x_{3}\left[\frac{5}{2}\left(g_{\mu \nu} g_{\lambda \rho}+g_{\mu \rho} g_{\nu \lambda}\right)+\right. \\
& \left.\frac{34}{24}\left(g_{\mu \nu} g_{\lambda \rho}+g_{\mu \lambda} g_{\nu \rho}+g_{\mu \rho} g_{\nu \lambda}\right)\right] I_{0}^{R}\left(M_{7 a}^{2}\right)+2 \text { permutations }
\end{aligned}
$$

The second diagram (Fig.7b) is given by: 


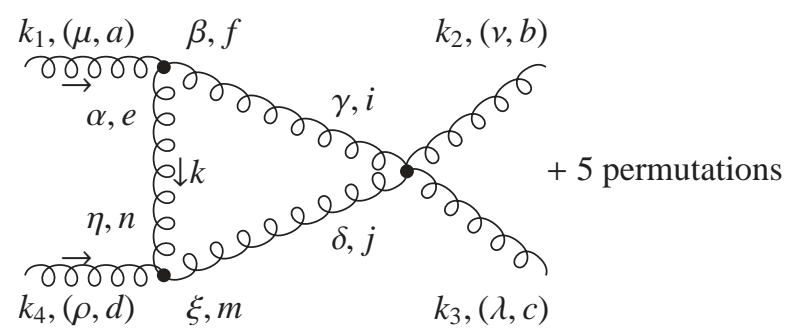

Fig.7b.

$$
\begin{aligned}
L(7 b)_{\mu \nu \lambda \rho}^{a b c d}= & \int \frac{d^{4} k}{(2 \pi)^{4}} g f^{a e f}\left[\left(k_{1}+k\right)_{\beta} g_{\mu \alpha}+\left(-k-k+k_{1}\right)_{\mu} g_{\alpha \beta}+\left(k-k_{1}-k_{1}\right)_{\alpha} g_{\beta \mu}\right] \frac{-i \delta^{e n} g^{\alpha \eta}}{k^{2}} \\
& g f^{d m n}\left[\left(k_{4}+k+k_{4}\right)_{\eta} g_{\rho \chi}+\left(-k-k_{4}-k\right)_{\rho} g_{\chi \eta}+\left(k-k_{4}\right)_{\chi} g_{\eta \rho}\right] \frac{-i \delta^{m j} g^{\chi \delta}}{\left(k+k_{4}\right)^{2}} \\
& (-i) g^{2}\left[f^{l i b} f^{l c j}\left(g_{\gamma \lambda} g_{\nu \delta}-g_{\gamma \delta} g_{\nu \lambda}\right)+f^{l i c} f^{l j b}\left(g_{\gamma \delta} g_{\lambda \nu}-g_{\gamma \nu} g_{\lambda \delta}\right)+\right. \\
& \left.f^{l i j} f^{l b c}\left(g_{\gamma \nu} g_{\delta \lambda}-g_{\gamma \lambda} g_{\delta \nu}\right)\right] \frac{-i \delta^{i f} g^{\gamma \beta}}{\left(k-k_{1}\right)^{2}}+5 \text { permutations } \\
= & g^{4} \int \frac{d^{4} k}{(2 \pi)^{4}} f^{a e f}\left[\left(k_{1}+k\right)_{\beta} g_{\mu \alpha}+\left(-k-k+k_{1}\right)_{\mu} g_{\alpha \beta}+\left(k-k_{1}-k_{1}\right)_{\alpha} g_{\beta \mu}\right] f^{d m e}\left[\left(k_{4}+k+k_{4}\right)^{\alpha} g_{\rho \chi}+\right. \\
& \left.\left(-k-k_{4}-k\right)_{\rho} g_{\chi}^{\alpha}+\left(k-k_{4}\right)_{\chi} g_{\rho}^{\alpha}\right]\left[f^{l f b} f^{l c m}\left(g_{\lambda}^{\beta} g_{\nu}^{\chi}-g^{\beta \chi} g_{\nu \lambda}\right)+f^{l f c} f^{l m b}\left(g^{\beta \chi} g_{\lambda \nu}-g_{\nu}^{\beta} g_{\lambda}^{\chi}\right)+\right. \\
& \left.f^{l f m} f^{l b c}\left(g_{\nu}^{\beta} g_{\lambda}^{\chi}-g_{\lambda}^{\beta} g_{\nu}^{\chi}\right)\right] \frac{1}{k^{2}\left(k+k_{4}\right)^{2}\left(k-k_{1}\right)^{2}}+5 \text { permutations }
\end{aligned}
$$

its divergent part reads:

$$
\begin{aligned}
& L(7 b)_{\mu \nu \lambda \rho ; i v}^{a b c d}=g^{4} \int \frac{d^{4} k}{(2 \pi)^{4}} f^{a e f} f^{d m e}\left[f^{l f b} f^{l c m}\left(g_{\lambda}^{\beta} g_{\nu}^{\chi}-g^{\beta \chi} g_{\nu \lambda}\right)+f^{l f c} f^{l m b}\left(g^{\beta \chi} g_{\lambda \nu}-g_{\nu}^{\beta} g_{\lambda}^{\chi}\right)+f^{l f m} f^{l b c}\left(g_{\nu}^{\beta} g_{\lambda}^{\chi}-g_{\lambda}^{\beta} g_{\nu}^{\chi}\right)\right] \\
& {\left[k_{\beta} g_{\mu \alpha}-2 k_{\mu} g_{\alpha \beta}+k_{\alpha} g_{\beta \mu}\right]\left[k^{\alpha} g_{\rho \chi}-2 k_{\rho} g_{\chi}^{\alpha}+k_{\chi} g_{\rho}^{\alpha}\right] \frac{1}{k^{2}\left(k+k_{4}\right)^{2}\left(k-k_{1}\right)^{2}}+5 \text { permutations }} \\
& =g^{4} \int \frac{d^{4} k}{(2 \pi)^{4}} f^{a e f} f^{d m e}\left[f^{l f b} f^{l c m}\left(g_{\lambda}^{\beta} g_{\nu}^{\chi}-g^{\beta \chi} g_{\nu \lambda}\right)+f^{l f c} f^{l m b}\left(g^{\beta \chi} g_{\lambda \nu}-g_{\nu}^{\beta} g_{\lambda}^{\chi}\right)+f^{l f m} f^{l b c}\left(g_{\nu}^{\beta} g_{\lambda}^{\chi}-g_{\lambda}^{\beta} g_{\nu}^{\chi}\right)\right] \\
& \int_{0}^{1} \int_{0}^{x_{1}} d x_{1} d x_{2} \frac{\Gamma(3)}{\Gamma(1) \Gamma(1) \Gamma(1)} \frac{\left[k_{\beta} g_{\mu \alpha}-2 k_{\mu} g_{\alpha \beta}+k_{\alpha} g_{\beta \mu}\right]\left[k^{\alpha} g_{\rho \chi}-2 k_{\rho} g_{\chi}^{\alpha}+k_{\chi} g_{\rho}^{\alpha}\right]}{\left[\left(1-x_{1}\right) k^{2}+\left(x_{1}-x_{2}\right)\left(k+k_{4}\right)^{2}+x_{2}\left(k-k_{1}\right)^{2}\right]^{3}}+5 \text { permutations } \\
& =2 g^{4} \int \frac{d^{4} k}{(2 \pi)^{4}} f^{a e f} f^{d m e}\left[f^{l f b} f^{l c m}\left(g_{\lambda}^{\beta} g_{\nu}^{\chi}-g^{\beta \chi} g_{\nu \lambda}\right)+f^{l f c} f^{l m b}\left(g^{\beta \chi} g_{\lambda \nu}-g_{\nu}^{\beta} g_{\lambda}^{\chi}\right)+f^{l f m} f^{l b c}\left(g_{\nu}^{\beta} g_{\lambda}^{\chi}-g_{\lambda}^{\beta} g_{\nu}^{\chi}\right)\right] \\
& \int_{0}^{1} \int_{0}^{x_{1}} d x_{1} d x_{2} \frac{k^{2} g_{\beta \mu} g_{\rho \chi}-k_{\beta} k_{\mu} g_{\rho \chi}-2 k_{\beta} k_{\rho} g_{\mu \chi}+4 k_{\mu} k_{\rho} g_{\beta \chi}+k_{\beta} k_{\chi} g_{\mu \rho}-2 k_{\mu} k_{\chi} g_{\beta \rho}-k_{\rho} k_{\chi} g_{\mu \beta}}{\left(k^{2}-M_{7 b}^{2}\right)^{3}}+ \\
& +5 \text { permutations }
\end{aligned}
$$

with $M_{7 b}=\left(x_{1}-x_{2}\right)\left(x_{1}-x_{2}-1\right) k_{4}^{2}+x_{2}\left(x_{2}-1\right) k_{1}^{2}$. Taking the relation $I_{0 \mu \nu}^{R}=\frac{1}{4} g_{\mu \nu} I_{0}^{R}$, we finally yield:

$$
\begin{aligned}
& L(7 b)_{\mu \nu \lambda \rho ; d i v}^{a b c d R}= 2 g^{4} f^{a e f} f^{d m e}\left[f^{l f b} f^{l c m}\left(g_{\lambda}^{\beta} g_{\nu}^{\chi}-g^{\beta \chi} g_{\nu \lambda}\right)+f^{l f c} f^{l m b}\left(g^{\beta \chi} g_{\lambda \nu}-g_{\nu}^{\beta} g_{\lambda}^{\chi}\right)+f^{l f m} f^{l b c}\left(g_{\nu}^{\beta} g_{\lambda}^{\chi}-g_{\lambda}^{\beta} g_{\nu}^{\chi}\right)\right] \\
& \int_{0}^{1} \int_{0}^{x_{1}} d x_{1} d x_{2}\left(g_{\beta \mu} g_{\rho \chi}-\frac{1}{4} g_{\beta \mu} g_{\rho \chi}-\frac{2}{4} g_{\beta \rho} g_{\mu \chi}+\frac{4}{4} g_{\mu \rho} g_{\beta \chi}+\frac{1}{4} g_{\beta \chi} g_{\mu \rho}-\frac{2}{4} g_{\mu \chi} g_{\beta \rho}-\frac{1}{4} g_{\rho \chi} g_{\mu \beta}\right) I_{0}\left(M_{7 b}\right) \\
&+5 \text { permutations } \\
& \text { (C37) }
\end{aligned}
$$

The third diagram (Fig.7c) is evaluated to be: 


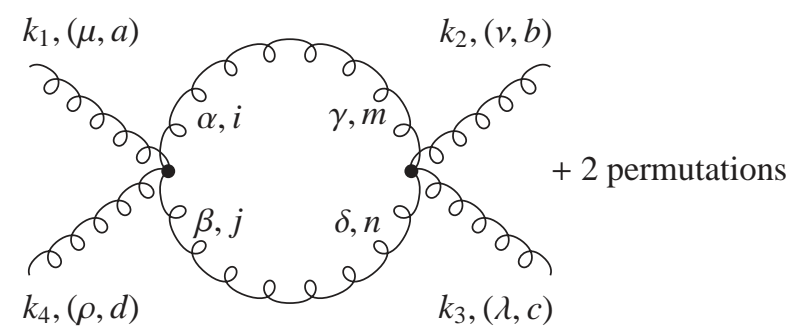

Fig.7c.

$$
\begin{aligned}
L(7 c)_{\mu \nu \lambda \rho}^{a b c d}= & \frac{1}{2} \int \frac{d^{4} k}{(2 \pi)^{4}}(-i) g^{2}\left[f^{e a i} f^{e j d}\left(g_{\mu \beta} g_{\alpha \rho}-g_{\mu \rho} g_{\alpha \beta}\right)+f^{e a j} f^{e d i}\left(g_{\mu \rho} g_{\beta \alpha}-g_{\mu \alpha} g_{\beta \rho}\right)+f^{e a d} f^{e i j}\left(g_{\mu \alpha} g_{\rho \beta}-g_{\mu \beta} g_{\rho \alpha}\right)\right] \\
& \times(-i) g^{2}\left[f^{f m b} f^{f c n}\left(g_{\gamma \lambda} g_{\nu \delta}-g_{\gamma \delta} g_{\nu \lambda}\right)+f^{f m c} f^{f n b}\left(g_{\gamma \delta} g_{\lambda \nu}-g_{\gamma \nu} g_{\lambda \delta}\right)+f^{f m n} f^{f b c}\left(g_{\gamma \nu} g_{\delta \lambda}-g_{\gamma \lambda} g_{\delta \nu}\right)\right] \\
& \times \frac{-i \delta^{i m} g^{\alpha \gamma}}{k^{2}} \frac{-i \delta^{j n} g^{\beta \delta}}{\left(k+k_{1}+k_{4}\right)^{2}}+2 \text { permutations } \\
= & \frac{1}{2} g^{4} \int \frac{d^{4} k}{(2 \pi)^{4}}\left[f^{e a i} f^{e j d}\left(g_{\mu \beta} g_{\alpha \rho}-g_{\mu \rho} g_{\alpha \beta}\right)+f^{e a j} f^{e d i}\left(g_{\mu \rho} g_{\beta \alpha}-g_{\mu \alpha} g_{\beta \rho}\right)+f^{e a d} f^{e i j}\left(g_{\mu \alpha} g_{\rho \beta}-g_{\mu \beta} g_{\rho \alpha}\right)\right] \\
& \times\left[f^{f i b} f^{f c j}\left(g_{\lambda}^{\alpha} g_{\nu}^{\beta}-g^{\alpha \beta} g_{\nu \lambda}\right)+f^{f i c} f^{f j b}\left(g^{\alpha \beta} g_{\lambda \nu}-g_{\nu}^{\alpha} g_{\lambda}^{\beta}\right)+f^{f i j} f^{f b c}\left(g_{\nu}^{\alpha} g_{\lambda}^{\beta}-g_{\lambda}^{\alpha} g_{\nu}^{\beta}\right)\right] \frac{1}{k^{2}\left(k+k_{1}+k_{4}\right)^{2}} \\
& +2 p e r m u t a t i o n s \\
= & \frac{1}{2} g^{4}\left[f^{e a i} f^{e j d}\left(g_{\mu \beta} g_{\alpha \rho}-g_{\mu \rho} g_{\alpha \beta}\right)+f^{e a j} f^{e d i}\left(g_{\mu \rho} g_{\beta \alpha}-g_{\mu \alpha} g_{\beta \rho}\right)+f^{e a d} f^{e i j}\left(g_{\mu \alpha} g_{\rho \beta}-g_{\mu \beta} g_{\rho \alpha}\right)\right] \\
& \times\left[f^{f i b} f^{f c j}\left(g_{\lambda}^{\alpha} g_{\nu}^{\beta}-g^{\alpha \beta} g_{\nu \lambda}\right)+f^{f i c} f^{f j b}\left(g^{\alpha \beta} g_{\lambda \nu}-g_{\nu}^{\alpha} g_{\lambda}^{\beta}\right)+f^{f i j} f^{f b c}\left(g_{\nu}^{\alpha} g_{\lambda}^{\beta}-g_{\lambda}^{\alpha} g_{\nu}^{\beta}\right)\right] \\
& \int_{0}^{1} d x_{1} \int \frac{d^{4} k}{(2 \pi)^{4}} \frac{\Gamma[2]}{\Gamma[1]^{2}} \frac{1}{\left(k^{2}-M_{7 c}^{2}\right)^{2}}+2 \text { permutations}
\end{aligned}
$$

with $M_{7 c}=x_{1}\left(x_{1}-1\right)\left(k_{1}+k_{4}\right)^{2}$. The divergent part is:

$$
\begin{aligned}
L(7 c)_{\mu \nu \lambda \rho ; i v}^{a b c d R}= & \frac{1}{2} g^{4}\left[f^{e a i} f^{e j d}\left(g_{\mu \beta} g_{\alpha \rho}-g_{\mu \rho} g_{\alpha \beta}\right)+f^{e a j} f^{e d i}\left(g_{\mu \rho} g_{\beta \alpha}-g_{\mu \alpha} g_{\beta \rho}\right)+f^{e a d} f^{e i j}\left(g_{\mu \alpha} g_{\rho \beta}-g_{\mu \beta} g_{\rho \alpha}\right)\right] \times \\
& {\left[f^{f i b} f^{f c j}\left(g_{\lambda}^{\alpha} g_{\nu}^{\beta}-g^{\alpha \beta} g_{\nu \lambda}\right)+f^{f i c} f^{f j b}\left(g^{\alpha \beta} g_{\lambda \nu}-g_{\nu}^{\alpha} g_{\lambda}^{\beta}\right)+f^{f i j} f^{f b c}\left(g_{\nu}^{\alpha} g_{\lambda}^{\beta}-g_{\lambda}^{\alpha} g_{\nu}^{\beta}\right)\right] } \\
& \int_{0}^{1} d x_{1} I_{0}\left(M_{7 c}\right)+2 \text { permutations }
\end{aligned}
$$

The fourth diagram (Fig.7d) is given by:

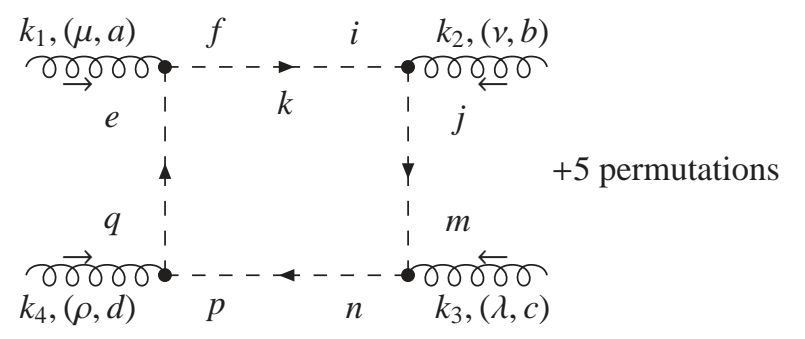

Fig.7d.

$$
\begin{aligned}
L(7 d)_{\mu \nu \lambda \rho}^{a b c d}= & -\int \frac{d^{4} k}{(2 \pi)^{4}}(-g) f^{a f e} k_{\mu} \frac{i \delta^{f i}}{k^{2}}(-g) f^{b j i}\left(k+k_{2}\right)_{\nu} \frac{i \delta^{j m}}{\left(k+k_{2}\right)^{2}}(-g) f^{c n m}\left(k+k_{2}+k_{3}\right)_{\lambda} \\
& \frac{i \delta^{n p}}{\left(k+k_{2}+k_{3}\right)^{2}} \times(-g) f^{d q p}\left(k-k_{1}\right)_{\rho} \frac{i \delta^{q e}}{\left(k-k_{1}\right)^{2}}
\end{aligned}
$$




$$
=-g^{4} f^{a i e} f^{b m i} f^{c p m} f^{d e p} \int \frac{d^{4} k}{(2 \pi)^{4}} \frac{k_{\mu}\left(k+k_{2}\right)_{\nu}\left(k+k_{2}+k_{3}\right)_{\lambda}\left(k-k_{1}\right)_{\rho}}{k^{2}\left(k+k_{2}\right)^{2}\left(k+k_{2}+k_{3}\right)^{2}\left(k-k_{1}\right)^{2}}
$$

so the divergent part is:

$$
\begin{aligned}
L(7 d)_{\mu \nu \lambda \rho ; d i v}^{a b c d}= & -g^{4} f^{\text {aie }} f^{b m i} f^{c p m} f^{d e p} \int \frac{d^{4} k}{(2 \pi)^{4}} \frac{k_{\mu} k_{\nu} k_{\lambda} k_{\rho}}{k^{2}\left(k+k_{2}\right)^{2}\left(k+k_{2}+k_{3}\right)^{2}\left(k-k_{1}\right)^{2}}+5 \text { permutations } \\
= & -g^{4} f^{\text {aie }} f^{b m i} f^{c p m} f^{d e p} \int_{0}^{1} d x_{1} \int_{0}^{x_{1}} d x_{2} \int_{0}^{x_{2}} d x_{3} \int \frac{d^{4} k}{(2 \pi)^{4}} \frac{\Gamma[4]}{\Gamma[1] \Gamma[1] \Gamma[1] \Gamma[1]} \frac{k_{\mu} k_{\nu} k_{\lambda} k_{\rho}}{\left(k^{2}-M_{7 d}^{2}\right)^{4}} \\
& +5 \text { permutations } \\
= & -6 g^{4} f^{\text {aie }} f^{b m i} f^{c p m} f^{d e p} \int_{0}^{1} d x_{1} \int_{0}^{x_{1}} d x_{2} \int_{0}^{x_{2}} d x_{3} I_{0 \mu \nu \lambda \rho}\left(M_{7 d}^{2}\right)+5 \text { permutations }
\end{aligned}
$$

where $M_{7 d}=\left(x_{1}-x_{2}\right)\left(x_{1}-x_{2}-1\right) k_{2}^{2}+\left(x_{2}-x_{3}\right)\left(x_{2}-x_{3}-1\right)\left(k_{2}+k_{3}\right)^{2}+x_{3}\left(x_{3}-1\right) k_{1}^{2}$. Using the relation

$$
I_{0 \mu \nu \rho \sigma}^{R}=\frac{1}{24}\left(g_{\mu \nu} g_{\rho \sigma}+g_{\mu \rho} g_{\nu \sigma}+g_{\mu \sigma} g_{\nu \rho}\right) I_{0}^{R}
$$

the divergent part becomes:

$$
\begin{aligned}
L(7 d)_{\mu \nu \lambda \rho ; d i v}^{a b c d R}= & -\frac{1}{4} g^{4} f^{a i e} f^{b m i} f^{c p m} f^{d e p}\left(g_{\mu \nu} g_{\lambda \rho}+g_{\mu \lambda} g_{\nu \rho}+g_{\mu \rho} g_{\nu \lambda}\right) \int_{0}^{1} d x_{1} \int_{0}^{x_{1}} d x_{2} \int_{0}^{x_{2}} d x_{3} I_{0}^{R}\left(M_{7 d}^{2}\right) \\
& +5 \text { permutations }
\end{aligned}
$$

The last diagram (Fig.7e) is evaluated to be

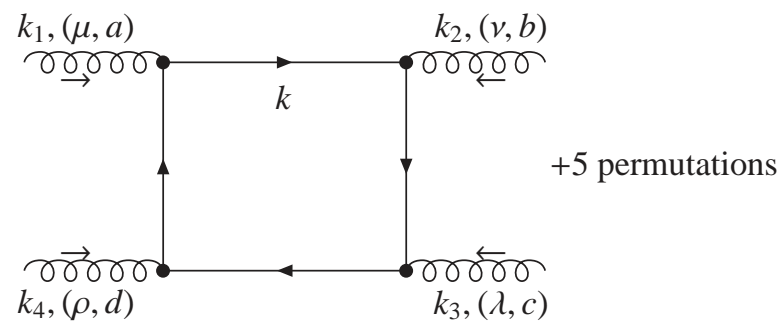

Fig.7e.

$$
\begin{aligned}
L(7 e)_{\mu \nu \lambda \rho}^{a b c d}= & -\operatorname{Tr} \int \frac{d^{4} k}{(2 \pi)^{4}} i g \gamma_{\mu} T^{a} \frac{i}{\not k-\not k_{4}-m} i g \gamma_{\rho} T^{d} \frac{i}{\not k+\not k k_{2}+\not k_{3}-m} i g \gamma_{\lambda} T^{c} \frac{i}{\not k+\not k}-m i g \gamma_{\nu} T^{b} \frac{i}{\not k-m} \\
& +5 \text { permutations } \\
= & -g^{4} \operatorname{Tr}\left(T^{a} T^{d^{2}} T^{c} T^{b}\right) \int \frac{d^{4} k}{(2 \pi)^{4}} \frac{\operatorname{tr}\left[\gamma_{\mu}\left(\not k-\not k k_{4}+m\right) \gamma_{\rho}\left(\not k+\not k k_{2}+\not k_{3}+m\right) \gamma_{\lambda}\left(\not k+\not k k_{2}+m\right) \gamma_{\nu}(\not k+m)\right]}{\left[\left(k-k_{4}\right)^{2}-m^{2}\right]\left[\left(k+k_{2}+k_{3}\right)^{2}-m^{2}\right]\left[\left(k+k_{2}\right)^{2}-m^{2}\right]\left[k^{2}-m^{2}\right]} \\
& +5 \text { permutations }
\end{aligned}
$$

the divergent part reads:

$$
\begin{aligned}
L(7 e)_{\mu \nu \lambda \rho ; d i v}^{a b c d}= & -g^{4} \operatorname{Tr}\left(T^{a} T^{d} T^{c} T^{b}\right) \int \frac{d^{4} k}{(2 \pi)^{4}} \frac{\operatorname{tr}\left(\gamma_{\mu} \not k \gamma_{\rho} \not k \gamma_{\lambda} \not k \gamma_{\nu} \not k\right)}{\left[\left(k-k_{4}\right)^{2}-m^{2}\right]\left[\left(k+k_{2}+k_{3}\right)^{2}-m^{2}\right]\left[\left(k+k_{2}\right)^{2}-m^{2}\right]\left[k^{2}-m^{2}\right]} \\
& +5 \text { permutations } \\
\sim & -g^{4} \operatorname{Tr}\left(T^{a} T^{d} T^{c} T^{b}\right) \int_{0}^{1} d x_{1} \int_{0}^{x_{1}} d x_{2} \int_{0}^{x_{2}} d x_{3} \int \frac{d^{4} k}{(2 \pi)^{4}} \frac{\Gamma[4]}{\Gamma[1] \Gamma[1] \Gamma[1] \Gamma[1]} \frac{\operatorname{tr}\left(\gamma_{\mu} \not k \gamma_{\rho} \not k \gamma_{\lambda} \not k \gamma_{\nu} \not k\right)}{\left(k^{2}-M_{7 e}^{2}\right)^{4}} \\
& +5 \text { permutations } \\
= & -24 g^{4} \operatorname{Tr}\left(T^{a} T^{d} T^{c} T^{b}\right) \int_{0}^{1} d x_{1} \int_{0}^{x_{1}} d x_{2} \int_{0}^{x_{2}} d x_{3} \int \frac{d^{4} k}{(2 \pi)^{4}} \frac{1}{\left(k^{2}-M_{7 e}^{2}\right)^{4}} \\
& \times\left(g_{\mu \nu} g_{\lambda \rho} k^{4}-g_{\mu \lambda} g_{\nu \rho} k^{4}+g_{\mu \rho} g_{\nu \lambda} k^{4}-2 g_{\mu \nu} k_{\lambda} k_{\rho} k^{2}-2 g_{\nu \lambda} k_{\rho} k_{\mu} k^{2}-2 g_{\lambda \rho} k_{\mu} k_{\nu} k^{2}\right. \\
& \left.-2 g_{\rho \mu} k_{\nu} k_{\lambda} k^{2}+8 k_{\mu} k_{\nu} k_{\lambda} k_{\rho}\right)+5 \text { permutations } \\
&
\end{aligned}
$$


where $M_{7 e}=m^{2}+\left(x_{1}-x_{2}\right)\left(x_{1}-x_{2}-1\right) k_{4}^{2}+\left(x_{2}-x_{3}\right)\left(x_{2}-x_{3}-1\right)\left(k_{2}+k_{3}\right)^{2}+x_{3}\left(x_{3}-1\right) k_{2}^{2}$. Using the relation $I_{0 \mu \nu}^{R}=\frac{1}{4} g_{\mu \nu} I_{0}^{R}$, we finally obtain:

$$
L(7 e)_{\mu \nu \lambda \rho ; d i v}^{a b c d R}=-8 g^{4} \operatorname{Tr}\left(T^{a} T^{d} T^{c} T^{b}\right)\left(g_{\mu \nu} g_{\lambda \rho}-2 g_{\mu \lambda} g_{\nu \rho}+g_{\mu \rho} g_{\nu \lambda}\right) \int_{0}^{1} d x_{1} \int_{0}^{x_{1}} d x_{2} \int_{0}^{x_{2}} d x_{3} I_{0}^{R}\left(M_{7 e}\right)+
$$

5 permutations

[1] Y.L. Wu, Int.J.Mod.Phys.A18: 5363 (2003), hep-th/0209021

[2] Y.L. Wu, Mod.Phys.Lett.A19: 2191 (2004), hep-th/0311082

[3] D. J. Gross and F. Wilczek, Phys.Rev.Lett30: 1343 (1973);

H. D. Politzer, Phys.Rev.Lett30: 1346 (1973);

T. Muta, Foundations of Quantum Chromodynamics, Appendix A and references therein, World Scientific, 1987.

[4] S. Tomonaga, Prog.Theor.Phys.Rev.Mod.Phys.1: 27 (1946)

[5] J. Schwinger, Phys.Rev.74: 1439 (1948); ibid. 75: 651 (1949); ibid. 76: 790 (1949).

[6] R.P. Feynman, Phys.Rev.76: 769 (1949).

[7] F.J. Dyson. Phys.Rev.75: 486, 1736 (1949).

[8] K.G. Wilson and J. Kogut, Phys.Repts.12C: 75 (1974).

[9] W. Heisenberg, Ann.d.Phys.32: 20 (1938).

[10] W. Pauli and F. Villars, Rev.Mod.Phys.21: 434 (1949).

[11] J. Schwinger, Phys.Rev.82:664 (1951).

[12] G.'t Hooft and M. Veltman, Nucl.Phys.B44: 189 (1972).

[13] K. G. Wilson,New Phenomena in Subnuclear Physics (Erice 1975), ed. A. Zichichi(Plenum, New York, 1977).

[14] F. del Aguila, A. Culatti, R. Munoz Tapia and M. Perez-Victori, Nucl.Phys.B537:561-585, 1999; M. Perez-Victori, Phys.Lett.B442:315-325,1998.

[15] Y.B. Dai and Y.L. Wu, Eur.Phys.J. C39:S1-S8 (2005).

[16] Y.L. Ma and Y.L. Wu, Int.J.Mod.Phys.A21:6383-6456 (2006).

[17] Y.L. Ma and Y.L. Wu, Phys.Lett.B647:427-430 (2007).

[18] L.D. Faddeev and V.N. Popov, Phys.Lett.B25:29-30 (1967); Abers and B.W. Lee, Phys.Rep9:1, 1973.

[19] G.'t Hooft, Nucl.Phys.B33: 173 (1971); ibid.B35: 167 (1971); G.'t Hooft and M.J.G. Veltman, Nucl.Phys.B44: 189 (1972); ibid.B50: 318 (1972).

[20] B.W. Lee, Phys.Rev.D5:823, (1972); B.W. Lee and J. Zinn-Justin, Phys.Rev.D5:3132, (1972); ibid. Phys.Rev.D5:3137, (1972); ibid. Phys.Rev.D5:3155, (1972); ibid. Phys.Rev.D7:1049, (1973); J. Zinn-Justin, Renormalization of gauge theories, Lectures at the 1974 Bonn Intern. Summer Institute for theoretical physics.

[21] H. Kluberg-Stern and J.B. Zuber, Phys.Rev.D12:482, (1974); ibid. Phys.Rev.D12:3159, (1975).

[22] M. Baker and C.K. Lee, Phys.Rev.D15:2201, (1977).

[23] Richard A. Brandt, Nucl.Phys.B116:413, (1976).

[24] A. A. Slavnov, Theor.Math.Phys.10:99-107, (1972); J. C. Taylor, Nucl.Phys.B33:436 (1971).

[25] C. Becchi, A. Rouet, R. Stora, Commun.Math.Phys.42:127-162 (1975); J. Zinn-Justin in Renormalization of gauge theories, Lectures at the 1974 Bonn Intern. Summer Institute for theoretical physics.

[26] G.'t Hooft, Nucl.Phys.B33: 173 (1971); S. D. Joglekar and B. W. Lee, Annals Phys.97:160 (1976).

[27] B.W.Lee in Methods in Field Theory, Les Houches 1975, ed R Balian and J. Zinn-Justin, North-Holland; B. W. Lee and J. Zinn-Justin Phys.Rev.D7:1049 (1973).

[28] See e.g.: John C. Collins Renormalization, Chap 7.1.1, Cambridge University Press, 1984.

[29] An interesting conjecture about dimension transmutation, see e.g.: C. T. Hill, hep-th/0510177, reference therein.

[30] W. Celmaster and R. J. Gonsalves, Phys.Rev.D20:142 (1979).

[31] P. Pascual and R. Tarrach, Nucl.Phys.B174:123 (1980).

[32] J.W. Cui, Y.Tang and Y.L. Wu, in preparation. 